Elsevier Editorial System(tm) for The Lancet Manuscript Draft

Manuscript Number:

Title: An international randomised controlled trial of ranibizumab compared with laser therapy for the treatment of very low birthweight infants with retinopathy of prematurity

Article Type: Article (Randomised Controlled Trial)

Corresponding Author: Professor Neil Marlow, DM FMedSci

Corresponding Author's Institution: UCL

First Author: Andreas Stahl, ND

Order of Authors: Andreas Stahl, ND; Domenico Lepore, MD; Alistair Fielder, FRCP; Brian Fleck, MD; James D Reynolds, MD; Michael F Chiang, MD; Jun Li, MD PhD; Melissa Liew, PhD; Rainer Maier, PhD; Qi Zhu, PhD; Neil Marlow, DM FMedSci

Abstract: Background: Current therapy does not eradicate ocular morbidity and visual disability following retinopathy of prematurity. Anti-vascular endothelial growth factor treatment provides a potentially new treatment. Methods: Infants with birth weight $<1,500 \mathrm{~g}$ meeting established criteria for ROP treatment were recruited in 87 neonatal and ophthalmology centres in 26 countries. We performed a randomised, multicentre, open-label, 3arm, parallel-group study evaluating efficacy and safety of intravitreal injection of ranibizumab $0 \cdot 2 \mathrm{mg}$ or ranibizumab $0 \cdot 1 \mathrm{mg}$ against laser therapy. The primary outcome was treatment success, defined as survival with no active retinopathy, unfavourable structural outcomes or the need for an additional treatment modality at or before 24 weeks. Findings: Treatment success occurred in 56/70 (80\%) infants receiving ranibizumab $0 \cdot 2 \mathrm{mg}$ compared with $57 / 76$ (75\%) receiving ranibizumab $0 \cdot 1 \mathrm{mg}$ and $45 / 68$ (66\%) infants following laser therapy. The odds ratio of a successful outcome following ranibizumab $0 \cdot 2 \mathrm{mg}$ compared with laser therapy was 2.19 (95\% confidence interval 0.99-4.82; $\mathrm{p}=0 \cdot 051$ ). One infant had an unfavourable structural outcome following ranibizumab $0 \cdot 2 \mathrm{mg}$, compared to five following ranibizumab $0 \cdot 1 \mathrm{mg}$ and seven after laser therapy. Ranibizumab $0 \cdot 2 \mathrm{mg}$ was effective in both Zone I and Zone II disease. Ranibizumab 0.1mg offered no advantage over $0 \cdot 2 \mathrm{mg}$. Death, serious and non-serious systemic and ocular adverse events were evenly distributed between the three groups.

Interpretation: In the treatment of retinopathy of prematurity, ranibizumab $0 \cdot 2 \mathrm{mg}$ was effective with fewer unfavourable ocular outcomes than laser therapy and with an acceptable short-term safety profile. Funding: Novartis Pharma; RAINBOW ClinicalTrials.gov number, NCT02375971. 


\title{
An international randomised controlled trial of ranibizumab compared with laser therapy for the treatment of very low birthweight infants with retinopathy of prematurity
}

Authors

Andreas Stahl MD1, Domenic Lepore $\mathrm{MD}^{2}$, Alistair Fielder FRCP ${ }^{3}$, Brian Fleck MD James D Reynolds MD $^{5}$, Michael F. Chiang MD ${ }^{6}$, Jun Li MD PhD ${ }^{7}$, Melissa Liew PhD ${ }^{7}$, Rainer Maier PhD ${ }^{7}, Q^{2}$ Zhu PhD $^{8}$, Neil Marlow DM ${ }^{9}$

${ }^{1}$ Department of Ophthalmology, University Medical Center Greifswald, Germany

${ }^{2}$ Catholic University of the Sacred Heart, Rome, Italy

${ }^{3}$ City University, London, UK

${ }^{4}$ Royal Hospital for Sick Children, University of Edinburgh, UK

${ }^{5}$ Ross Eye Institute, Jacobs School of Medicine and Biomedical Sciences, University at Buffalo, Buffalo, USA

${ }^{6}$ Casey Eye Institute, Oregon Health \& Science University, Oregon, USA

${ }^{7}$ Novartis Pharma AG, Basel, Switzerland

${ }^{8}$ China Novartis Institutes for BioMedical Research Company Ltd

${ }^{9}$ UCL Elizabeth Garrett Anderson Institute for Women's Health, University College London, London, UK

Corresponding Author:

\author{
Andreas Stahl \\ Department of Ophthalmology, University of Greifswald, Germany \\ Ferdinand-Sauerbruch-Straße \\ 17475 Greifswald, Germany \\ Email: andreas.stahl@uni-greifswald.de \\ Phone: +49 3834865900 \\ Fax: +493834865902
}

Key words:

Infant, Preterm; Retinopathy of Prematurity; Anti-VEGF treatment; Randomised controlled trial;

\section{Authors' contribution:}

The Study was conceived and designed by the authors in conjunction with Novartis. The Study was funded and managed by Novartis, with supervision by the authors, who formed the Protocol Steering Committee. The statistical analysis plan was agreed by all authors and undertaken by Qi Zhu. All contributors had full access to the data. The first draft of the manuscript was written by Neil Marlow with equal contributions from all the authors who were not Novartis employees. All authors have agreed the final version and submission to The Lancet.

\section{Conflicts of Interest statement:}

$A S, D L, A F, B F, J D R, M F C$ and NM received personal fees and travel reimbursement from Novartis Pharma AG during the design and execution of the trial. No fees were paid during the analysis and write up of the study. AS declares personal fees from Novartis, Bayer, Allergan, Orphan Europe, and Boehringer Ingelheim outside the submitted work. AF declares personal fees from Orphan Europe, MC declares personal fees from Clarity Medical Systems and Inteleretina, NM declares personal fees from Shire and GSK, all outside the submitted work. JL, ML, RM and ZQ are employees of Novartis Pharma AG. 
NM receives a proportion of funding from the Department of Health's NIHR Biomedical Research Centres funding scheme at UCLH/UCL. MFC is supported by National Institutes of Health grant P30EY10572 (Bethesda, MD), and by unrestricted departmental funding from Research to Prevent Blindness (New York, NY).

Word count:

Abstract $\quad 239$

Main text 2976

Tables 3

Figures 2

References 33 


\section{Research in Context}

\section{Evidence before this study}

Retinopathy of prematurity (ROP) is the primary cause of blindness in $28,300-45,600$ premature infants per year worldwide. Over the past 25 years treatment has been possible to halt the disease and prevent blindness using laser photocoagulation. This does, however, not eliminate ocular morbidity, which remains of concern.

Uncontrolled retinal neovascularisation (which drives the clinical progression of the disease) is provoked by local secretion of vascular endothelial growth factor (VEGF) in ischaemic retinal tissue. Anti-VEGF agents may thus be used to target uncontrolled retinal neovascularization in ROP. A first trial showed that bevacizumab, an anti-VEGF antibody, may prevent the need for retreatment in a defined US population with severe ROP. Since then, without research evidence of either long term benefit or risk and without attempts at finding the appropriate dose, off label anti-VEGF use has become widespread being the first and sometimes only treatment option in in many settings across the world.

Today, several aspects of the use of anti-VEGF agents remain unanswered: ocular efficacy, the appropriate drug and dose, the need for re-treatment, and the possibility of long-term systemic effects from sustained suppression of systemic VEGF in a developing infant.

\section{Added value of this study}

RAINBOW demonstrates that ranibizumab, an established anti-VEGF agent in adult practice, is an effective alternative to laser in commonly treated patient groups with ROP. Its use appears to be associated with better eye outcomes and it has an acceptable short term safety profile. Follow up is underway to determine vision outcomes to 5 years of age. In comparison to other anti-VEGF agents the suppression of systemic VEGF levels appears to be limited with ranibizumab. Importantly, RAINBOW investigated two ranibizumab doses to collect data on anti-VEGF dose finding in ROP.

\section{Implications}

Intravitreal injection of ranibizumab may be considered as a novel treatment for ROP. Disease recurrence, however, must be monitored closely as it is more common with anti-VEGF agents and can occur later compared to laser. The relative mild effects on systemic VEGF levels appear advantageous for ranibizumab over other anti-VEGF agents. It remains too early to determine definitive vision outcomes or to confirm long term systemic safety. Both will be addressed in the ongoing RAINBOW extension trial. 


\begin{abstract}
Background: Current therapy does not eradicate ocular morbidity and visual disability following retinopathy of prematurity. Anti-vascular endothelial growth factor treatment provides a potentially new treatment.
\end{abstract}

Methods: Infants with birth weight $<1,500 \mathrm{~g}$ meeting established criteria for ROP treatment were recruited in 87 neonatal and ophthalmology centres in 26 countries. We performed a randomised, multicentre, open-label, 3-arm, parallel-group study evaluating efficacy and safety of intravitreal injection of ranibizumab $0.2 \mathrm{mg}$ or ranibizumab $0.1 \mathrm{mg}$ against laser therapy. The primary outcome was treatment success, defined as survival with no active retinopathy, unfavourable structural outcomes or the need for an additional treatment modality at or before 24 weeks.

Findings: Treatment success occurred in 56/70 (80\%) infants receiving ranibizumab $0 \cdot 2 \mathrm{mg}$ compared with 57/76 (75\%) receiving ranibizumab $0 \cdot 1 \mathrm{mg}$ and $45 / 68$ (66\%) infants following laser therapy. The odds ratio of a successful outcome following ranibizumab $0 \cdot 2 \mathrm{mg}$ compared with laser therapy was $2 \cdot 19$ (95\% confidence interval 0.99-4.82; $\mathrm{p}=0.051)$. One infant had an unfavourable structural outcome following ranibizumab $0 \cdot 2 \mathrm{mg}$, compared to five following ranibizumab $0 \cdot 1 \mathrm{mg}$ and seven

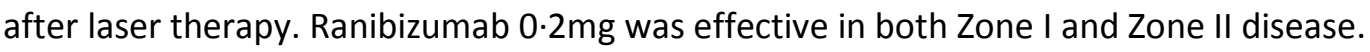
Ranibizumab $0.1 \mathrm{mg}$ offered no advantage over $0.2 \mathrm{mg}$. Death, serious and non-serious systemic and ocular adverse events were evenly distributed between the three groups.

Interpretation: In the treatment of retinopathy of prematurity, ranibizumab $0 \cdot 2 \mathrm{mg}$ was effective with fewer unfavourable ocular outcomes than laser therapy and with an acceptable short-term safety profile.

Funding: Novartis Pharma; RAINBOW ClinicalTrials.gov number, NCT02375971.

239 words 


\section{Introduction}

Retinopathy of prematurity (ROP) is a disease of the developing retina. Mild forms of ROP resolve spontaneously with few sequelae, but severe ROP can progress to retinal detachment, vision impairment and blindness. Ablation of the peripheral retina with cryotherapy,(1) or confluent laser photocoagulation (2) reduces but does not eliminate ocular morbidity. Annually, 28,300-45,600 infants worldwide are diagnosed with irreversible visual impairment from ROP.(3)

Anti-vascular endothelial growth factor (anti-VEGF) agents injected into the vitreous are widely used for vasoproliferative disease in adult ophthalmic practice and present a new opportunity for ROP treatment. To date, the largest clinical trial of anti-VEGF in preterm infants compared the efficacy of intravitreal bevacizumab with laser therapy in a defined US population. There were fewer retreatments in the bevacizumab group. The difference was significant only for ROP located in retina Zone I (the most posterior region of the retina).(4) This study was followed by several case series,(57) small trials $(8-10)$ and two small dosing studies. $(11,12)$ In clinical practice, anti-VEGF agents are increasingly being used worldwide to treat ROP.

Several aspects of anti-VEGF therapy in ROP remain unanswered: ocular efficacy, the appropriate drug and dose, the need for re-treatment, and the possibility of long-term systemic effects.(13-17) In light of these unanswered questions and the wide spread off label use of anti-VEGF treatment in ROP, there is an urgent need to explore the use of anti-VEGF agents under defined trial conditions, not only in the US and Europe but also in other countries where neonatal care may be more variable. The need is especially pressing in such lower resource settings where an estimated $60 \%$ of the world's ROP occurs,(3) and anti-VEGF agents are being widely used as the primary treatment. This not surprising for several reasons: compared to laser, intravitreal injection is relatively simple to administer, has significantly shorter administration time, requires fewer resources, less user expertise and often no anaesthesia is given. 
RAINBOW, RAnibizumab compared with laser therapy for the treatment of INfants BOrn prematurely With retinopathy of prematurity, was designed to evaluate the efficacy and safety of two doses of ranibizumab against conventional laser therapy in a randomised open-label study that recruited infants worldwide, with a broad scope of ROP stages. Here, we report the outcomes of RAINBOW from enrolment through 24 weeks post treatment. 


\section{Methods}

Study objectives: The primary objective was to investigate whether intravitreal ranibizumab $0 \cdot 2 \mathrm{mg}$ has superior efficacy to laser therapy in the treatment of ROP, as defined by survival without active ROP, unfavourable structural outcomes, or the need for a treatment modality other than that assigned ("treatment switch"), in both eyes, up to 24 weeks after starting investigational treatment. Active ROP was defined as vessel dilatation ("plus disease") in at least two quadrants, or extraretinal vessels extending from the retina into the vitreous. Unfavourable structural outcomes included retrolental membrane obscuring the view of the posterior pole; substantial temporal retinal vessel dragging causing abnormal structural features/macular ectopia; posterior retinal fold involving the macula; or retinal detachment involving the macula.

Secondary objectives were to evaluate the efficacy of ranibizumab $0.1 \mathrm{mg}$, the requirement of any post-baseline treatment, ocular and systemic safety of intravitreal ranibizumab, pharmacokinetics of intravitreal ranibizumab, and systemic VEGF levels (Supplementary Appendix).

Study oversight: The study was conducted in accordance with the Declaration of Helsinki. The study protocol was reviewed and approved by an Independent Ethics Committee or Institutional Review Board at each contributing centre. Parents or guardians provided written informed consent. An independent data and safety monitoring board oversaw the safety of study participants.

Role of the Funding Source: This is a Novartis sponsored and funded study. The authors who are not Novartis employees have received no payment for their role in analysis, interpretation and writing of the manuscript. They have full access to the data, which is owned by Novartis. The authors take full responsibility for the decision to submit for publication.

Study design: The RAINBOW study was a randomised, open label, 3-arm, parallel group, superiority study evaluating the efficacy and safety of intravitreal ranibizumab compared to laser therapy for the treatment of ROP. Eligible infants were randomised 1:1:1 to receive bilateral intravitreal ranibizumab $0.2 \mathrm{mg}$, intravitreal ranibizumab $0.1 \mathrm{mg}$ or laser therapy at baseline (Day 1 , treatment 
phase). The follow-up phase was until Day 169 (24 weeks) when the infant exited the study and became eligible to join the RAINBOW extension study in which long-term efficacy and safety will be studied to 5 years of age.

Study population: Preterm infants with a birth weight of less than 1,500g and with a diagnosis of bilateral ROP Zone I Stage $1+, 2+, 3$ or 3+, or Zone II Stage 3+, or aggressive posterior ROP (AP-ROP) were enrolled.(18) Zone II Stage 2+ was not included as treatment is controversial in some countries.(19) Exclusion criteria included ocular and neurological co-morbidities that might result in confounding visual impairment, and active ocular infection within 5 days before investigational treatment.

Randomisation and Treatment: Random allocation was performed using interactive response technology, stratified by ROP zone of the worst eye, as this characteristic might impact treatment outcome, and by geographic region to reflect variations in practice. Region 1 comprised countries with a neonatal mortality $<5$ per 1,000 live births in 2012, and Region 2 countries with higher neonatal mortality rates(20). The allocated treatment was applied to both eyes, and signs of disease persistence or recurrence were used by investigators to determine whether additional treatment was needed (Supplementary Appendix). Under the protocol, in the laser therapy group, supplementary treatment to "skip lesions" was allowed up to Day 11. In the ranibizumab groups, up to two additional treatments with ranibizumab were allowed in each eye at a minimum of 28-day intervals. Only a switch to a different treatment modality was considered as treatment failure.

Ocular outcomes: The retina was assessed at baseline and at each study visit up to Day 169. Examinations were performed using indirect ophthalmoscopy and, where available, by wide-field retinal imaging. Seventy five of the 87 study centres performed retinal imaging. These images were transmitted to a central reading centre for documentation.

Pharmacology: We adopted a sparse sampling approach to pharmacokinetics, sampling within 24 hours of baseline treatment, and around 15 and 29 days after treatment. The concentration of 
ranibizumab in serum samples was determined using ELISA assay. The concentration of VEGF was determined in K3-EDTA plasma samples by an electrochemiluminescence sandwich immunoassay (ECLA).

Statistical analysis: We assumed a failure event rate for ranibizumab $0 \cdot 2 \mathrm{mg}$ of $6 \cdot 5 \%$ and laser $42 \cdot 4 \%$ in Zone I, and $5 \cdot 1 \%$ and $12.5 \%$ in Zone II, respectively, which was similar to that observed in the Bevacizumab Eliminates the Angiogenic Threat of Retinopathy of Prematurity (BEAT-ROP) trial for the recurrence of ROP at 54 weeks postmenstrual age,(4) and that each patient had a $45 \%$ and $55 \%$ probability of ROP in Zones I and II. At least 80 evaluable patients per treatment arm (100 enrolled patients with an estimated drop-out rate of $20 \%$ ) were expected to provide more than $90 \%$ power to show superiority of ranibizumab $0.2 \mathrm{mg}$ against laser therapy (the primary objective of the study). Slow enrolment and recruitment challenges led the sponsor to change this target after consultation with the European Medicine Agency (EMA). 60 patients per treatment arm (48 evaluable patients assuming a drop-out rate of $20 \%$ ) were expected to provide more than $80 \%$ power to show superiority of ranibizumab $0 \cdot 2 \mathrm{mg}$ against laser therapy. This minimum target replaced the original target but it was agreed that additional patients above this new target should be enrolled, until preplanned closure of enrolment on $30^{\text {th }}$ June 2017.

The Cochran-Mantel Haenszel test was used to test the primary outcome as recruitment was stratified by zone of ROP. The pre-specified level of significance was two-sided alpha $=0.05$ for superiority of ranibizumab $0 \cdot 2 \mathrm{mg}$ against laser therapy. A sequential test strategy was used to minimise the risk of Type 1 errors caused by multiple comparisons. Significance testing of secondary outcomes was only performed if the test of primary outcome was significant. The Mantel-Haenszel odds ratio with $95 \%$ confidence intervals $(\mathrm{Cl})$ is presented for each comparison. Efficacy analysis was by intention to treat. 
Data Sharing: Study results have been disclosed as required by corresponding regulations and are publicly available on ClinicalTrials.gov, the EU Clinical Trial Registry, and the Novartis Clinical Trial Results websites. 


\section{Results}

Baseline characteristics: Eighty-seven centres in 26 countries enrolled infants (Figure S1 in the Supplementary Appendix) between December 2015 and June 2017. Of 230 screened infants, 225 entered into RAINBOW and were allocated equally between the three study groups. Of these, 218 received baseline treatment (Day 1), and 201 infants completed the study (Figure 1).

The median gestational age for participants at birth was 26 weeks (range 23-32 weeks). Gestational age was lower in the ranibizumab $0 \cdot 2 \mathrm{mg}$ group (median: 25 weeks) than the other two groups (median: 26 weeks, respectively) and lower in Region 1 (median 24 weeks) than in Region 2 (28 weeks). Most other baseline characteristics were well balanced between study groups (Table 1).

Categorisation of ROP was similarly distributed across the treatment groups at baseline (Supplementary Appendix Table S1). ROP was present in Zone I in $86(38 \cdot 2 \%)$ infants and in Zone II in $138(61 \cdot 3 \%)$ infants. Most infants had Stage 3+ disease, 135 (60.0\%) in Zone II and 37 (16.4\%) in Zone I. AP-ROP was present in $30(13 \cdot 3 \%)$ infants, 29 of whom had Zone I disease. Wide-field retinal imaging was performed at least once in $88 \%$ infants.

Efficacy outcomes: The primary efficacy outcome is known for 214 infants (Figure 1). Treatment success occurred in 45/68 (66\%) of infants who received laser therapy (Table 2), 56/70 (80\%) of infants who received ranibizumab $0 \cdot 2 \mathrm{mg}$ (odds ratio compared to laser: $2 \cdot 19$ (95\% Cl 0.99-4.82); $\mathrm{p}=0 \cdot 051$ ), and $57 / 76(75 \%)$ of infants who received ranibizumab $0 \cdot 1 \mathrm{mg}$ (odds ratio compared to laser: $1 \cdot 57(95 \% \mathrm{Cl} 0 \cdot 76-3 \cdot 26))$.

Three infants in the ranibizumab $0.1 \mathrm{mg}$ group had active ROP at Week 24 , comprising plus disease in two or more quadrants but with no extra-retinal vessels extending from the retina. No patients in the other groups had active disease. One infant had an unfavourable structural outcome following ranibizumab $0.2 \mathrm{mg}$, compared to five following ranibizumab $0.1 \mathrm{mg}$ and seven after laser therapy. (Supplementary Appendix Table S2). 
An exploratory adjusted logistic regression analysis for the primary outcome correcting for three potential confounders, gestational age, geographic region and infant sex was performed; the odds ratio in favour of ranibizumab $0 \cdot 2 \mathrm{mg}$ versus laser treatment were $2 \cdot 32(95 \% \mathrm{Cl} 1 \cdot 04-5 \cdot 16)$.

Overall, treatment success was higher for all three groups in Zone II compared with Zone I and the gradient of effect remained in favour of ranibizumab (Table 2). Among the two ranibizumab groups, full peripheral vascularisation assessed by indirect ophthalmoscopy occurred by Day 169 in 28 (38\%) infants in the $0.2 \mathrm{mg}$ group and $21(27 \%)$ infants in the $0.1 \mathrm{mg}$ group.

Sixty one infants received additional post-baseline treatments: $23(31 \%)$ in the ranibizumab $0 \cdot 2 \mathrm{mg}$ group; 24 (31\%) in the ranibizumab 0.1mg group; and 14 (19\%) in the laser therapy group (Figure 2). In the laser group this was in addition to the treatment of skip lesions in 11 infants before Day 11 but including one infant that received laser on Day 12. Including treatment to skip lesions, 22 (30\%) infants in the laser arm needed additional post-baseline treatment (laser or switch to ranibizumab). Twelve infants in each of the ranibizumab groups had one or more ranibizumab re-treatments and achieved a successful primary outcome. The median interval between baseline and first retreatment was 55 (range: $29-111$ ) days in the ranibizumab 0.2mg group and 57 (range: $30-128$ ) days in the ranibizumab $0 \cdot 1 \mathrm{mg}$ group.

Safety outcomes: Death, serious and non-serious systemic adverse events (AEs) were evenly distributed between the three groups (Supplementary Appendix Tables S3, S4, S5). All events were from complications of preterm birth. Four deaths occurred in each group, including one ranibizumab $0.1 \mathrm{mg}$-treated infant in whom the investigator suspected that death was related to the study drug or procedure. The infant died at home following a feed within 24 hours of treatment, and was classified as 'fatal respiratory failure' (Supplementary Appendix Table S3). Serious and non-serious ocular AEs were similarly distributed between the three groups (Supplementary Appendix Tables S6 and S7), and the more clinically relevant events that occurred are summarised in Table S8. 
One infant (ranibizumab $0.2 \mathrm{mg}$ ) had a moderate cataract in one eye noted on Day 28 , as a result of lens damage by the injecting needle. Cataract surgery was not performed during the study. One infant (ranibizumab $0.1 \mathrm{mg}$ ) developed unilateral endophthalmitis and orbital infection 6 days after intravitreal injection. The infant had a history of bilateral conjunctivitis and of left periocular staphylococcal infection. Prophylactic topical antibiotic was given to the left eye for 3 days immediately prior to intravitreal injection. Ipsilateral conjunctivitis and orbital infection presented 4 days after injection and endophthalmitis was evident at 6 days. Vitrectomy was performed at 8 weeks. The retinal structural outcome was unfavourable.

There were no significant differences between the study groups in body length, head circumference, knee to heel length, weight or blood pressure from baseline to Days 85 or 169 (Supplementary Appendix Table S9).

Ranibizumab pharmacokinetics and plasma VEGF levels: Median serum ranibizumab and plasma VEGF levels within 24 hours of, and 14 days and 28 days after intravitreal injection are shown in Table 3. Serum ranibizumab levels decayed from immediately post baseline to $57 \%$ ( $0 \cdot 2 \mathrm{mg}$ group) and $78 \%$ ( $0 \cdot 1 \mathrm{mg}$ group) after 14 days, and $13-14 \%$ of baseline by 28 days (Supplementary Appendix Figure S2). Plasma VEGF levels were reduced by $37-48 \%$ in all three treatment groups 14 days after baseline treatment and returned towards baseline levels by Day 29 (Supplementary Appendix Figure S3). 


\section{Discussion}

In the RAINBOW study, ranibizumab was an effective and well-tolerated treatment for ROP in a range of settings around the world. The proportion of infants with successful outcome was greater following intravitreal ranibizumab $0.2 \mathrm{mg}$ compared with laser photocoagulation but did not reach statistical significance $(p=0.051)$. In particular, ranibizumab $0.2 \mathrm{mg}$ resulted in fewer eyes with unfavourable structural outcomes than either ranibizumab $0.1 \mathrm{mg}$ or laser therapy. Ranibizumab $0 \cdot 1 \mathrm{mg}$ appeared to offer no advantage over the use of $0 \cdot 2 \mathrm{mg}$. Further safety data and functional outcomes to 5 years of age will be ascertained during the ongoing extension study. The observed systemic safety profile was as expected in a preterm population, and ocular AEs were consistent with the established profile for ranibizumab in adults.

RAINBOW included ROP in all Zone II locations, including mid and anterior zone II. The efficacy of ranibizumab was similar to laser in both Zone I and Zone II disease. In all three treatment groups the highest risk of unfavourable outcome occurred in Zone I disease, as has been reported in other trials $(1,4,21)$. Switching to alternative forms of treatment was frequent in all three groups. This may have been partly caused by the study design as the rules for additional treatments were set at a low threshold to minimize the risk of visual impairment and applied to both laser and ranibizumab arms.

Although RAINBOW and the BEAT-ROP trial both evaluated anti-VEGF agents, direct comparisons should be approached with caution as the two trials have very different protocols. RAINBOW included all forms of ETROP Type 1 ROP apart from stage 2+ in Zone II from an international group of hospitals; in contrast BEAT-ROP only included stage 3+ disease in Zone I and posterior Zone II in a single region. In RAINBOW outcomes were evaluated per patient 24 weeks after first treatment (at a mean age of 60 weeks postmenstrual age (PMA)), whereas in BEAT-ROP ascertainment was by each eye to 54 weeks PMA (and occurred between 50-70 weeks PMA). RAINBOW used a composite primary outcome that included survival without active ROP, unfavourable structural outcomes, or 
treatment switch; in contrast BEAT-ROP used recurrence of retinal neovascularisation requiring retreatment. Thus, the two studies are not directly comparable.

In the Early Treatment of Retinopathy of Prematurity Randomized Trial, (21) 9.1\% of eyes had unfavourable structural outcomes, which compares to $1.5 \%$ eyes (1.5\% infants) in RAINBOW following ranibizumab $0 \cdot 2 \mathrm{mg}$ and $6 \%$ of eyes ( $11 \%$ of infants) following laser. The efficacy of ranibizumab with fewer unfavourable structural outcomes compared to laser needs to be weighed against the two serious ocular complications cataract (1) and endophthalmitis (1).

In RAINBOW, additional treatments were administered throughout the follow-up period. Regular follow-up eye examinations are therefore required for at least 24 weeks after ranibizumab treatment. Median time to recurrence requiring additional treatment was 8 weeks, indicating that this time frame is particularly important during follow-up examinations, but much later recurrences after anti-VEGF therapy may also occur.(22)

Ranibizumab is a fully humanised monoclonal antigen-binding 48kDa Fab antibody fragment.(23) In adults, the serum half-life of ranibizumab following intravitreal administration is approximately 9 days following intravitreal injection, probably due to slow egress from the vitreous.(24) RAINBOW is the first study to measure ranibizumab pharmacokinetics in preterm infants. Serum ranibizumab levels fell slowly with detectable but much reduced levels at Day 29 (13 and 14\%). In contrast, bevacizumab, a 149 kDa monoclonal antibody, has a serum half-life of 21 days following intravitreal injection in preterm infants.(25)

VEGF-mediated angiogenesis is essential for the development of a number of tissues, $(26,27)$ but little is known about the effects of VEGF suppression in preterm infants. We measured plasma rather than serum VEGF levels as this avoids VEGF release from platelets during thrombolysis and better reflects true free VEGF.(28) Although blood concentrations do not prove systemic safety, in RAINBOW they suggest ranibizumab treatment and laser therapy have similar effects on plasma levels of VEGF. The reduced plasma VEGF levels observed after laser therapy may be due to ablation 
of VEGF-secreting tissue. $(25,29)$ Plasma VEGF levels were reduced in all three RAINBOW groups and showed a similar profile over the study period. In CARE-ROP, no systematic directional effect of ranibizumab on plasma VEGF was observed.(11) In contrast, following intravitreal bevacizumab in preterm infants serum VEGF levels were reduced to $50 \%$ of those treated with laser for at least 60 days.(25)

In summary, ranibizumab $0 \cdot 2 \mathrm{mg}$ was as effective and safe in the treatment of active ROP as laser photocoagulation and was associated with better short-term ocular outcomes. Compared with laser treatment, the potential for procedural complications and the need for regular clinical follow up after ranibizumab treatment must be balanced against fewer adverse structural outcomes. 


\section{References:}

1. Multicenter trial of cryotherapy for retinopathy of prematurity. Preliminary results.

Cryotherapy for Retinopathy of Prematurity Cooperative Group. Archives of Ophthalmology

(Chicago, III : 1960). 1988; 106: 471-9.

2. Revised indications for the treatment of retinopathy of prematurity: results of the early treatment for retinopathy of prematurity randomized trial. Archives of Ophthalmology (Chicago, III : 1960). 2003; 121: 1684-94.

3. Blencowe H, Lawn JE, Vazquez T, Fielder A, Gilbert C. Preterm-associated visual impairment and estimates of retinopathy of prematurity at regional and global levels for 2010. Pediatric Research. 2013; 74 Suppl 1: 35-49.

4. Mintz-Hittner HA, Kennedy KA, Chuang AZ. Efficacy of intravitreal bevacizumab for stage 3+ retinopathy of prematurity. The New England Journal of Medicine. 2011; 364: 603-15.

5. Huang $Q$, Zhang $Q$, Fei $P$, et al. Ranibizumab Injection as Primary Treatment in Patients with Retinopathy of Prematurity: Anatomic Outcomes and Influencing Factors. Ophthalmology. 2017; 124: 1156-64.

6. Hwang CK, Hubbard GB, Hutchinson AK, Lambert SR. Outcomes after Intravitreal Bevacizumab versus Laser Photocoagulation for Retinopathy of Prematurity: A 5-Year Retrospective Analysis. Ophthalmology. 2015; 122: 1008-15.

7. Lyu J, Zhang Q, Chen CL, et al. Recurrence of Retinopathy of Prematurity After Intravitreal Ranibizumab Monotherapy: Timing and Risk Factors. Investigative Ophthalmology \& Visual Science. 2017; 58: 1719-25. 
8. Lepore D, Quinn GE, Molle F, et al. Follow-up to Age 4 Years of Treatment of Type 1 Retinopathy of Prematurity Intravitreal Bevacizumab Injection versus Laser: Fluorescein Angiographic Findings. Ophthalmology. 2018; 125: 218-26.

9. Karkhaneh R, Khodabande A, Riazi-Eafahani M, et al. Efficacy of intravitreal bevacizumab for zone-II retinopathy of prematurity. Acta Ophthalmologica. 2016; 94: e417-20.

10. Zhang $\mathrm{G}$, Yang $\mathrm{M}$, Zeng J, et al. Comparison of intravitreal injection of ranibizumab versus laser therapy for zone II treatment-requiring retinopathy of prematurity. Retina (Philadelphia, Pa). 2017; 37: 710-7.

11. Stahl A, Krohne TU, Eter N, et al. Comparing Alternative Ranibizumab Dosages for Safety and Efficacy in Retinopathy of Prematurity: A Randomized Clinical Trial. JAMA Pediatrics. 2018; 172: 27886.

12. Wallace DK, Kraker RT, Freedman SF, et al. Assessment of Lower Doses of Intravitreous Bevacizumab for Retinopathy of Prematurity: A Phase 1 Dosing Study. JAMA Ophthalmology. 2017;135: 654-6.

13. Darlow BA, Ells AL, Gilbert CE, Gole GA, Quinn GE. Are we there yet? Bevacizumab therapy for retinopathy of prematurity. Archives of Disease in Childhood Fetal and Neonatal Edition. 2013; 98: F170-4.

14. Kennedy KA, Mintz-Hittner HA. Medical and developmental outcomes of bevacizumab versus laser for retinopathy of prematurity. Journal of AAPOS: the official publication of the American Association for Pediatric Ophthalmology and Strabismus. 2018; 22: 61-5.e1.

15. Lien R, Yu MH, Hsu KH, et al. Neurodevelopmental Outcomes in Infants with Retinopathy of Prematurity and Bevacizumab Treatment. PloS One. 2016; 11: e0148019. 
16. Morin J, Luu TM, Superstein R, et al. Neurodevelopmental Outcomes Following Bevacizumab Injections for Retinopathy of Prematurity. Pediatrics. 2016; 137: e20153218

17. Sankar MJ, Sankar J, Chandra P. Anti-vascular endothelial growth factor (VEGF) drugs for treatment of retinopathy of prematurity. The Cochrane Database of Systematic Reviews. 2018; 1: CD009734.

18. The International Classification of Retinopathy of Prematurity revisited. Archives of Ophthalmology (Chicago, III: 1960). 2005; 123: 991-9.

19. Wilkinson AR, Haines L, Head K, Fielder AR. UK retinopathy of prematurity guideline. Early Human Development. 2008; 84: 71-4.

20. World Health Statistics 2014: WHO http://apps.who.int/iris/bitstream/handle/10665/112738/9789240692671 eng.pdf;jsessionid=A60B 920F2CC95F44769825BE02B10541? sequence=1. (Accessed 6 March 2019)

21. Good WV. Final results of the Early Treatment for Retinopathy of Prematurity (ETROP) randomized trial. Transactions of the American Ophthalmological Society. 2004; 102: 233-48; and discussion 48-50.

22. Mintz-Hittner HA, Geloneck MM, Chuang AZ. Clinical Management of Recurrent Retinopathy of Prematurity after Intravitreal Bevacizumab Monotherapy. Ophthalmology. 2016; 123: 1845-55.

23. Ferrara N, Damico L, Shams N, Lowman H, Kim R. Development of ranibizumab, an antivascular endothelial growth factor antigen binding fragment, as therapy for neovascular age-related macular degeneration. Retina (Philadelphia, Pa). 2006; 26: 859-70.

24. Xu L, Lu T, Tuomi L, et al. Pharmacokinetics of ranibizumab in patients with neovascular agerelated macular degeneration: a population approach. Investigative Ophthalmology \& Visual Science. 2013; 54: 1616-24. 
25. Kong L, Bhatt AR, Demny AB, et al. Pharmacokinetics of bevacizumab and its effects on serum VEGF and IGF-1 in infants with retinopathy of prematurity. Investigative Ophthalmology \& Visual Science. 2015; 56: 956-61.

26. Tinning AR, Jensen BL, Johnsen I, Chen D, Coffman TM, Madsen K. Vascular endothelial growth factor signaling is necessary for expansion of medullary microvessels during postnatal kidney development. American Journal of Physiology Renal physiology. 2016; 311: F586-99.

27. Woik N, Kroll J. Regulation of lung development and regeneration by the vascular system. Cellular and molecular life sciences: CMLS. 2015; 72: 2709-18.

28. Walz JM, Boehringer D, Deissler HL, et al. Pre-Analytical Parameters Affecting Vascular Endothelial Growth Factor Measurement in Plasma: Identifying Confounders. PloS One. 2016; 11: e0145375.

29. Yalin Imamoglu E, Gunay M, Gursoy T, et al. Effect of laser photocoagulation on plasma levels of VEGF-A, VEGFR-2, and Tie2 in infants with retinopathy of prematurity. Journal of AAPOS: the official publication of the American Association for Pediatric Ophthalmology and Strabismus. 2014; 18: 466-70. 


\section{Figure Legends:}

Figure 1: CONSORT diagram detailing enrolment, randomization and follow up of the 230 infants screened for the RAINBOW trial

Figure 2: Progress of VLBW infants with ROP through additional post baseline treatments in RAINBOW up to 29 days, when ranibizumab re-treatment was first allowed, and 169 days (primary outcome). The flow chart shows the number of infants receiving at least one extra treatment, either allowed in the protocol $\left({ }^{*}\right)$ or treatment switches, which contributed to the primary outcome (shaded grey). Infants may have received one or more additional treatments in one or both eyes. Note laser to skip lesions was allowed as part of the baseline treatment but are included as they represent extra treatment episodes. 
Table 1: Demographics and baseline characteristics of 225 very low birthweight infants entered into the three trial groups in RAINBOW

\begin{tabular}{|c|c|c|c|}
\hline & $\begin{array}{l}\text { Ranibizumab } 0 \cdot 2 \mathrm{mg} \\
n=74\end{array}$ & $\begin{array}{l}\text { Ranibizumab } 0 \cdot 1 \mathrm{mg} \\
\qquad \mathrm{n}=77\end{array}$ & $\begin{array}{l}\text { Laser therapy } \\
\qquad \mathrm{n}=74\end{array}$ \\
\hline \multicolumn{4}{|c|}{ Geographical region ${ }^{(a)}, \mathrm{n}(\%)$} \\
\hline Region 1 & $45(60 \cdot 8)$ & $45(58 \cdot 4)$ & $44(59 \cdot 5)$ \\
\hline Region 2 & $29(39 \cdot 2)$ & $32(41 \cdot 6)$ & $30(40 \cdot 5)$ \\
\hline \multicolumn{4}{|l|}{ Sex, $n(\%)$} \\
\hline Male & $33(44 \cdot 6)$ & $37(48 \cdot 1)$ & $37(50 \cdot 0)$ \\
\hline Female & $41(55 \cdot 4)$ & $40(51 \cdot 9)$ & $37(50 \cdot 0)$ \\
\hline \multicolumn{4}{|c|}{ Mother's race or ethnic origin, $n(\%)$} \\
\hline Caucasian & $43(58 \cdot 1)$ & $45(58 \cdot 4)$ & $45(60 \cdot 8)$ \\
\hline Black & 0 & $4(5 \cdot 2)$ & $3(4 \cdot 1)$ \\
\hline Asian & $27(36 \cdot 5)$ & $22(28 \cdot 6)$ & $23(31 \cdot 1)$ \\
\hline Other & $4(5 \cdot 4)$ & $6(7 \cdot 8)$ & $3(4 \cdot 1)$ \\
\hline \multicolumn{4}{|c|}{ Gestational age in weeks } \\
\hline Median (range) & $25(23-32)$ & $26(23-32)$ & $26(23-32)$ \\
\hline Region $1 \operatorname{med}(r)$ & $24(23-30)$ & $25(23-29)$ & $24(23-31)$ \\
\hline Region $2 \operatorname{med}(r)$ & $28(24-32)$ & $28(24-32)$ & $28(24-32)$ \\
\hline \multicolumn{4}{|c|}{ Gestational age category in weeks, $\mathrm{n}(\%)$} \\
\hline$</=24$ & $32(43 \cdot 2)$ & $22(28 \cdot 6)$ & $29(39 \cdot 2)$ \\
\hline$>24-<27$ & $18(24 \cdot 3)$ & $21(27 \cdot 3)$ & $17(23 \cdot 0)$ \\
\hline
\end{tabular}




\begin{tabular}{|c|c|c|c|}
\hline$>/=27$ & $24(32 \cdot 4 \%)$ & $34(44 \cdot 2 \%)$ & $28(37 \cdot 8 \%)$ \\
\hline \multicolumn{4}{|l|}{ Birth weight (grams) } \\
\hline Mean (SD), & $791(244)$ & $886(299)$ & $831(284)$ \\
\hline z score mean (SD) & $-.49(1 \cdot 18)$ & $-.52(1.04)$ & $-.56(1 \cdot 14)$ \\
\hline \multicolumn{4}{|l|}{ Plurality, n (\%) } \\
\hline Singleton & $52(70 \cdot 3)$ & $55(71 \cdot 4)$ & $48(64 \cdot 9)$ \\
\hline Multiple & $18(24 \cdot 4)$ & $18(23 \cdot 4)$ & $18(24 \cdot 3)$ \\
\hline Missing & $4(5 \cdot 4)$ & $4(5 \cdot 2)$ & $8(10 \cdot 8)$ \\
\hline \multicolumn{4}{|c|}{ Postmenstrual age at baseline treatment in weeks } \\
\hline Median (range) & $\begin{array}{c}36 \cdot 7 \\
(30 \cdot 3-51 \cdot 9)\end{array}$ & $\begin{array}{c}36 \cdot 9 \\
(31 \cdot 9-54 \cdot 9)\end{array}$ & $\begin{array}{c}36 \cdot 6 \\
(30 \cdot 6-55 \cdot 3)\end{array}$ \\
\hline
\end{tabular}

(a) Geographical region 1 = neonatal mortality $<5$ per 1,000 live births, region $2=$ greater neonatal mortality (WHO 2014) 
Table 2: Primary efficacy outcome and prespecified subgroup analysis of 225 very low birthweight infants entered into the three trial groups in RAINBOW

\begin{tabular}{|c|c|c|c|}
\hline & $\begin{array}{c}\text { Ranibizumab } \\
0.2 \mathrm{mg}\end{array}$ & $\begin{array}{c}\text { Ranibizumab } \\
0.1 \mathrm{mg}\end{array}$ & Laser therapy \\
\hline Patients entered into RAINBOW & 74 & 77 & 74 \\
\hline Patients with known primary outcome ${ }^{(a)}$ & $n=70$ & $n=76$ & $n=68$ \\
\hline Treatment success ${ }^{(b)}, \mathrm{n}(\%)$ & $56(80)$ & $57(75)$ & $45(66 \cdot 2)$ \\
\hline \multicolumn{4}{|l|}{ Reason for failing primary objective ${ }^{(c)}$} \\
\hline Active ROP present ${ }^{(d)}$ & 0 & 3 & 0 \\
\hline Unfavourable structural outcome ${ }^{(e)}$ & 1 & 5 & 7 \\
\hline Treatment switch & 11 & 13 & 18 \\
\hline Death of infant & 4 & 4 & 4 \\
\hline \multicolumn{4}{|c|}{ Pre-specified subgroup analyses } \\
\hline \multicolumn{4}{|l|}{ Primary outcome by ROP Zone, n (\%) } \\
\hline Zone I & $19 / 28(67.9)$ & $21 / 30(70 \cdot 0)$ & $14 / 23(60 \cdot 9)$ \\
\hline Zone II & $37 / 42(88 \cdot 1)$ & $36 / 46(78 \cdot 3)$ & $31 / 45(68.9)$ \\
\hline \multicolumn{4}{|l|}{ Primary outcome by gestation } \\
\hline$</=24$ weeks & $22 / 29(75 \cdot 9)$ & $17 / 22(77 \cdot 3)$ & $12 / 27(44 \cdot 4)$ \\
\hline$>24-<27$ weeks & $16 / 18(88 \cdot 9)$ & $18 / 21(85 \cdot 7)$ & $9 / 15(60 \cdot 0)$ \\
\hline$>/=27$ weeks & $18 / 23(78 \cdot 3)$ & $22 / 33(66 \cdot 7)$ & $24 / 26(92 \cdot 3)$ \\
\hline Primary outcome by Region ${ }^{(\mathrm{f})}, \mathrm{n}(\%)$ & & & \\
\hline
\end{tabular}




\begin{tabular}{|llll|}
\hline Region 1 & $36 / 42(85 \cdot 7)$ & $37 / 45(82 \cdot 2)$ & $26 / 42(61 \cdot 9)$ \\
Region 2 & $20 / 28(71 \cdot 4)$ & $20 / 31(64 \cdot 5)$ & $19 / 26(73 \cdot 1)$ \\
Primary outcome by infant sex, $\mathbf{n}(\%)$ & & & \\
Male & $28 / 33(84 \cdot 8)$ & $26 / 36(72 \cdot 2)$ & $19 / 35(54 \cdot 3)$ \\
Female & $28 / 37(75 \cdot 7)$ & $31 / 40(77 \cdot 5)$ & $26 / 33(78 \cdot 8)$ \\
\hline
\end{tabular}

(a) See Fig 1

(b) Alive, without treatment switch, without either unfavourable structural outcome or active ROP at day 169

(c) Infants can have multiple events.

(d) Three infants with active ROP at day 169 had vessel dilatation (plus disease) but no extra-retinal vessels.

(e) Unfavourable structural outcome in either eye, any one of: retrolental membrane obscuring the view of the posterior pole; substantial temporal retinal vessel dragging causing abnormal structural features / macular ectopia; posterior retinal fold involving the macula; retinal detachment involving the macula.

(f) Geographical Region $1=$ neonatal mortality $<5$ per 1,000 live births, Region $2=$ greater infant mortality (WHO 2014). 
Table 3: Serum ranibizumab levels following intraocular injection in very low birthweight infants and plasma VEGF levels in all three trial groups in RAINBOW.

\begin{tabular}{|c|c|c|c|}
\hline & Ranibizumab $0.2 \mathrm{mg}$ & Ranibizumab $0.1 \mathrm{mg}$ & Laser therapy \\
\hline \multicolumn{4}{|c|}{ Serum ranibizumab, $\mathrm{pg} / \mathrm{mL}$} \\
\hline Day 1 median (IQR) (a) & $7,820(2,000-23,200)$ & $4,350(382-12,100)$ & - \\
\hline Day 15 median (IQR) & $4,440(2,450-8,130)$ & $3,400(2,515-5,215)$ & - \\
\hline Day 29 median (IQR) & $1,070(705-1,730)$ & $566(303-1,060)$ & - \\
\hline \multicolumn{4}{|l|}{ Plasma VEGF, pg/mL } \\
\hline Day 1 median (IQR) (b) & $136(78-414)$ & $130(81-388)$ & $136(79-288)$ \\
\hline Day 15 median (IQR) & $71.8(54-124)$ & $67(37-156)$ & $86.1(56-230)$ \\
\hline Day 29 median (IQR) & $89(74-105)$ & $140(97-209)$ & $123(63-181)$ \\
\hline
\end{tabular}


Fig 1

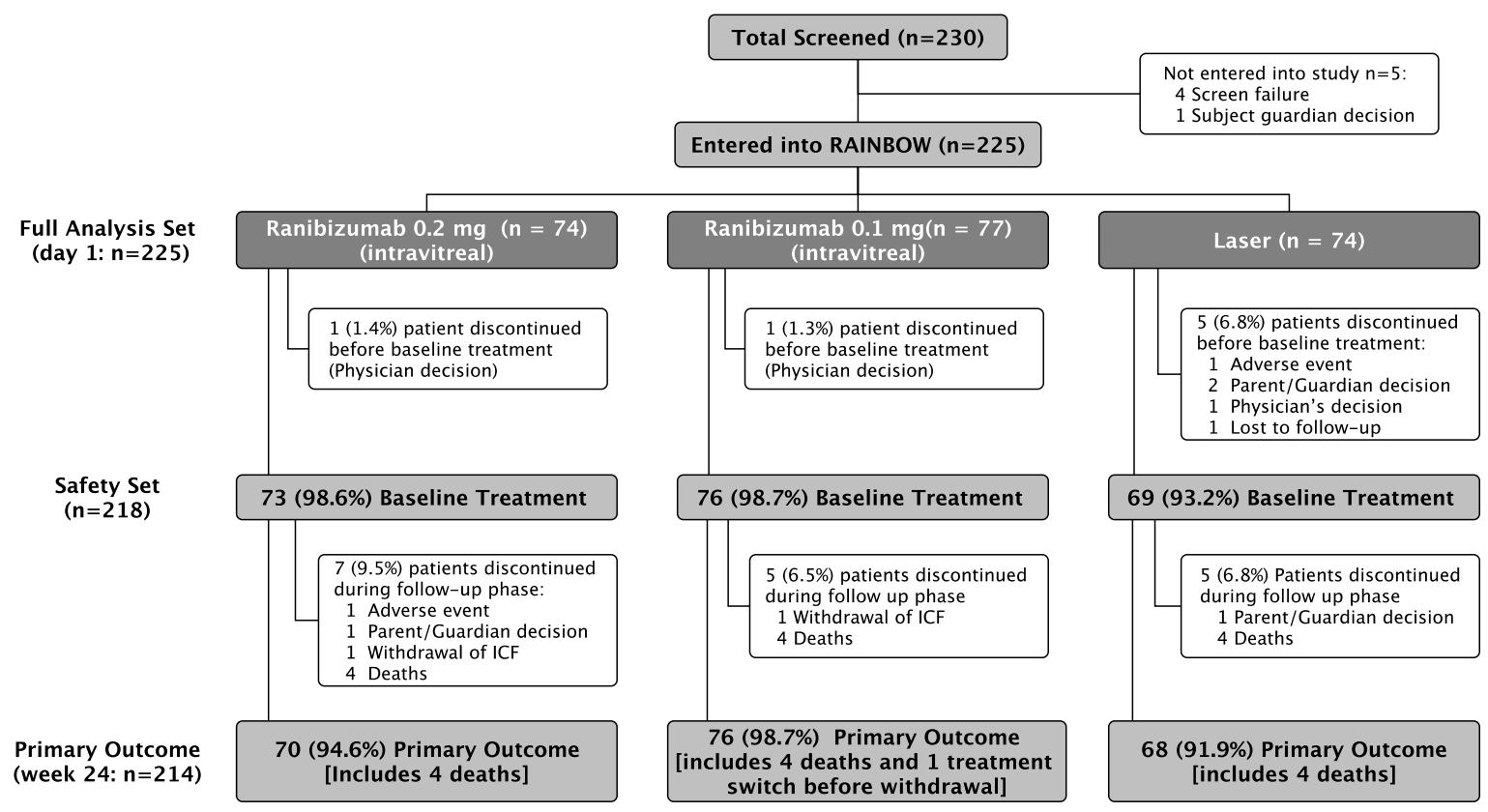


Fig 2

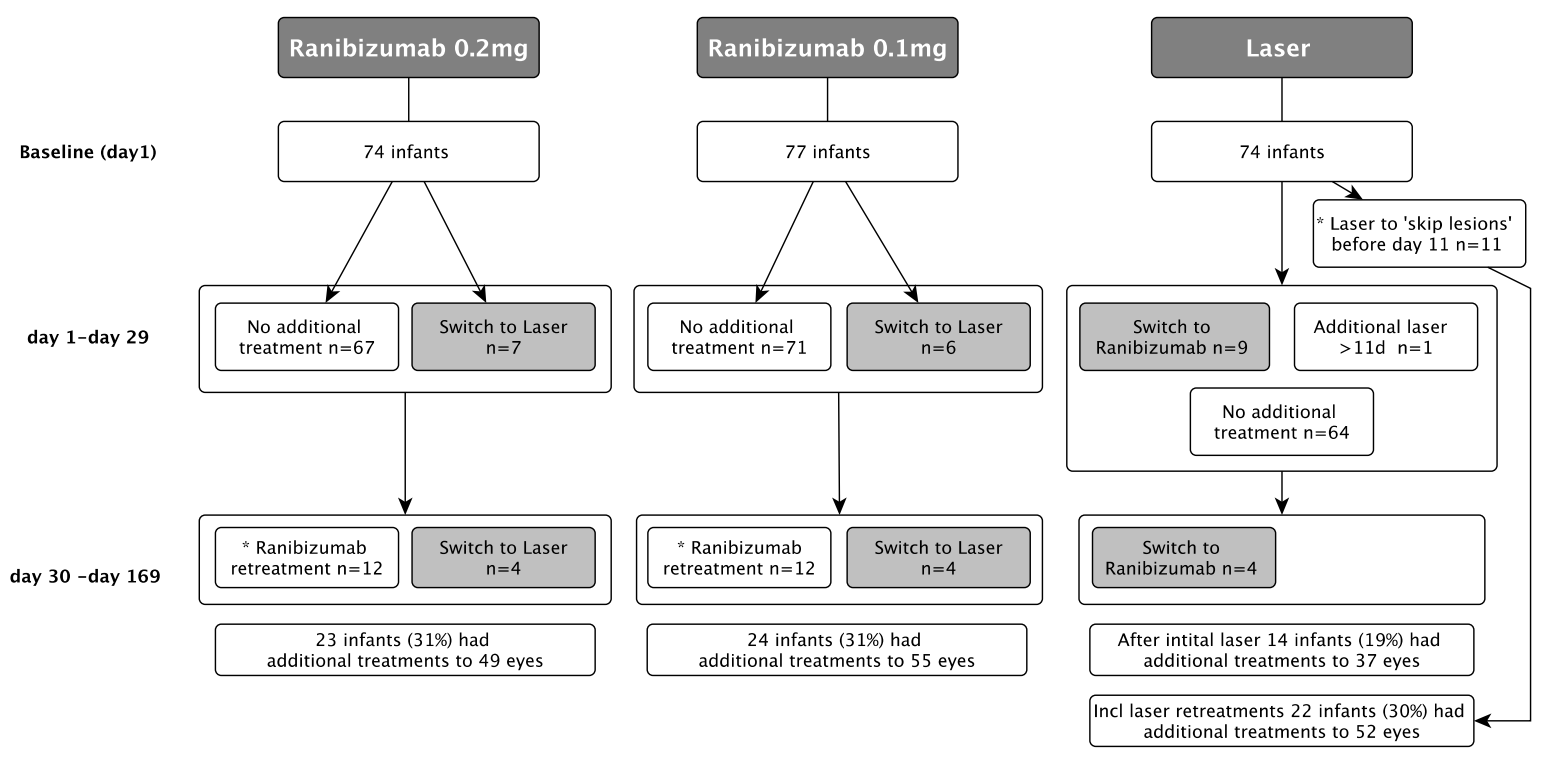


Web Appendix
Click here to d

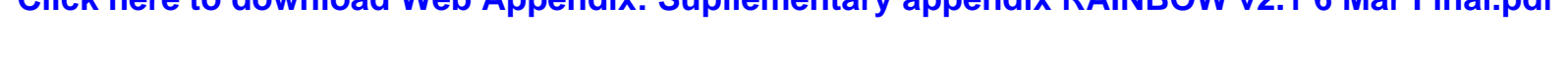

\author{
Web Appendix
Click here to download Web Appendix: Supllementary appendix RAINBOW v2.1 6 Mar Final.pdf
} (5) - 


\section{(b) NOVARTIS}

Global Clinical Development - General Medicine

RFB002/Ranibizumab

Clinical Trial Protocol CRFB002H2301

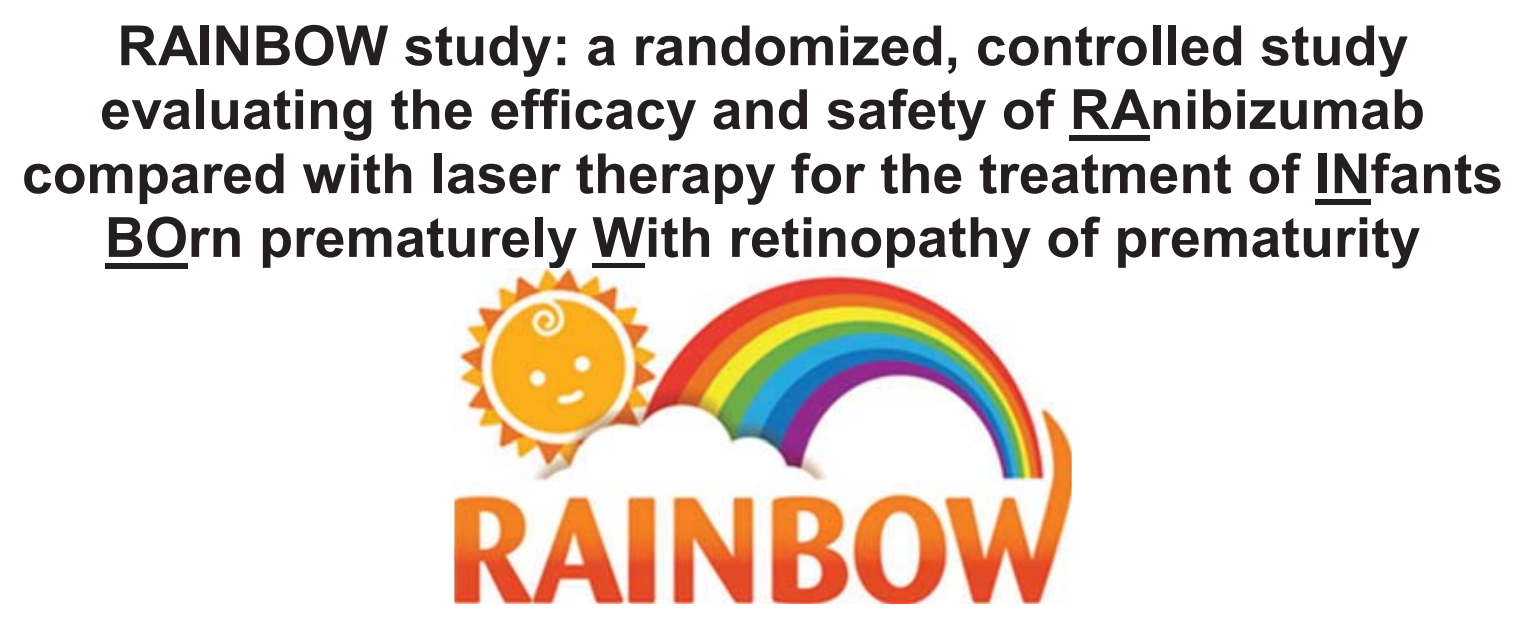

Document type: $\quad$ Amended Protocol Version

EUDRACT number: $\quad$ 2014-003041-10

Version number: $\quad$ v02 (Clean)

Development phase: 3

Release date: $\quad$ 01-Feb-2017

Property of Novartis

Confidential

May not be used, divulged, published, or otherwise disclosed without the consent of Novartis

Clinical Trial Protocol Template Version 2.0, November 2013 


\section{Table of contents}

Table of contents

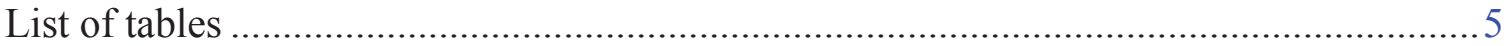

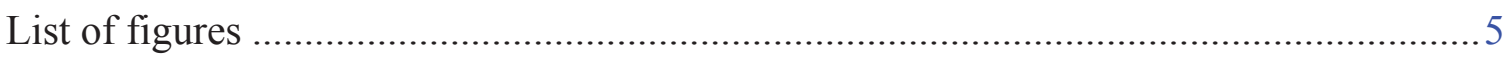

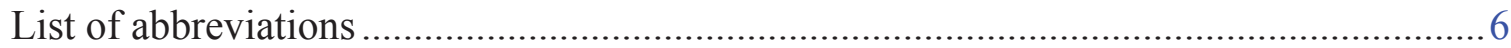

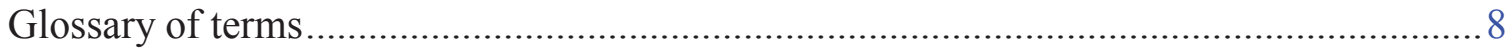

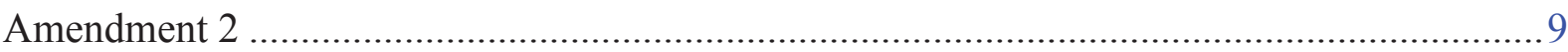

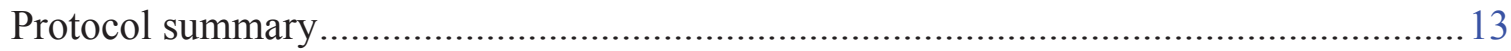

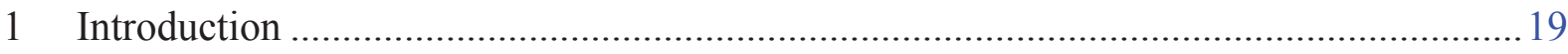

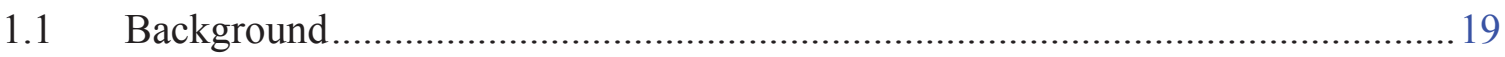

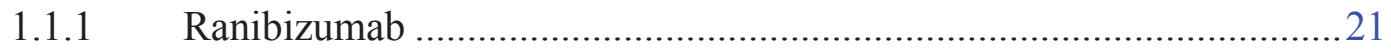

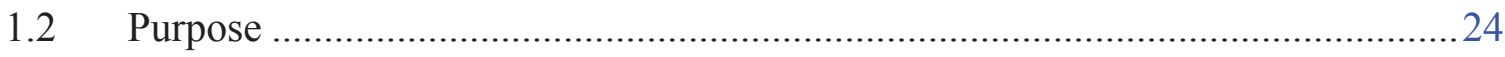

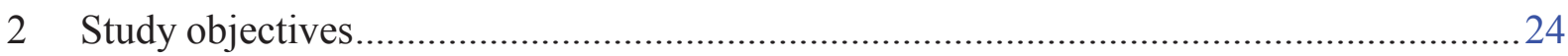

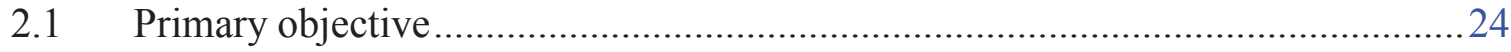

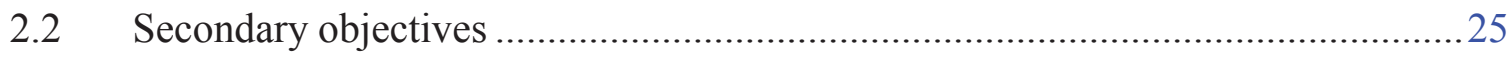

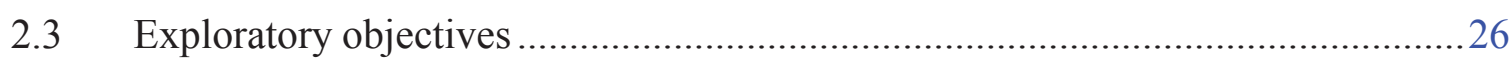

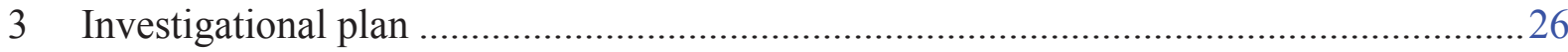

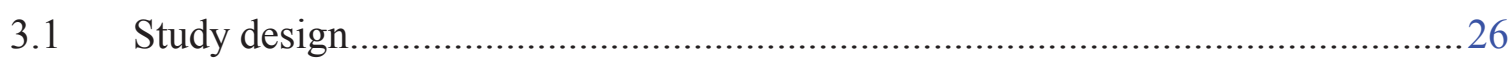

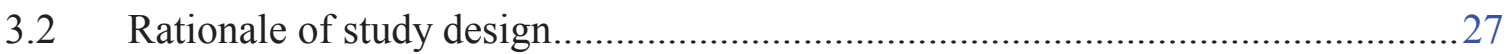

3.3 Rationale of dose/regimen, route of administration, and duration of treatment ....27

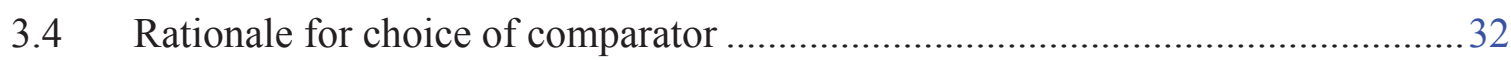

3.5 Purpose and timing of interim analyses/design adaptations ................................ 32

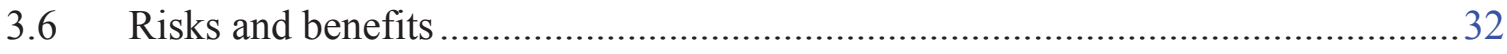

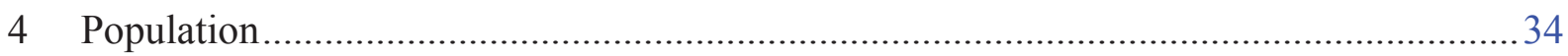

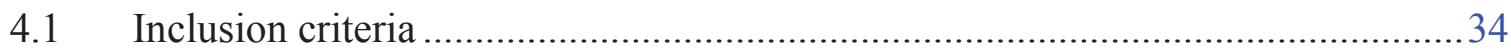

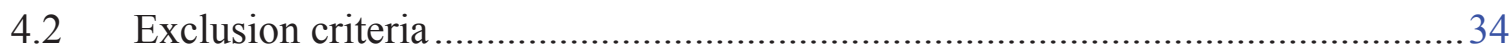

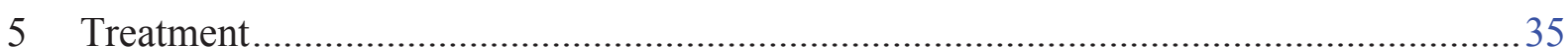

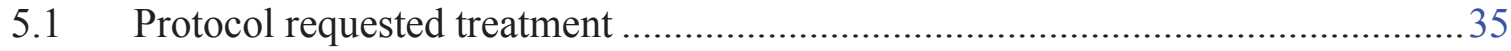

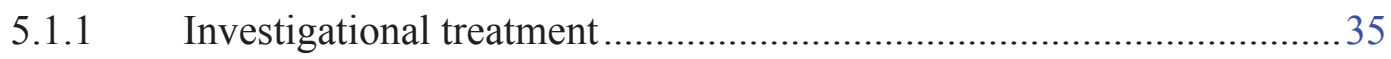

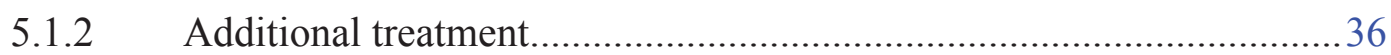

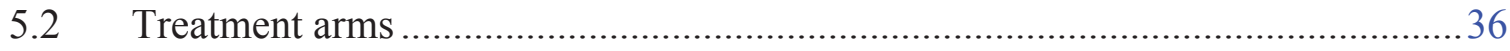

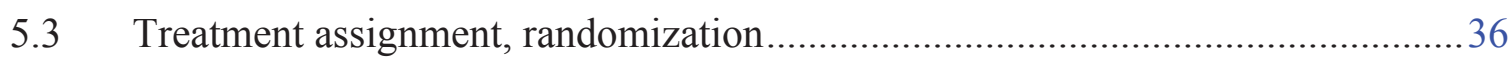

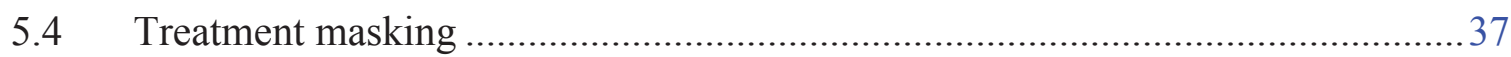

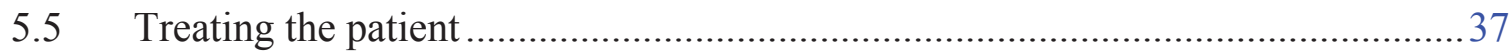

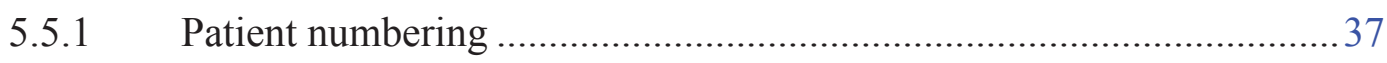

5.5.2 Dispensing the investigational treatment ............................................... 37 


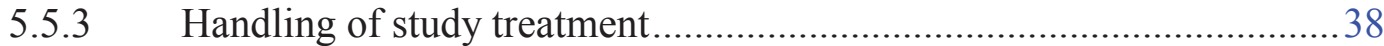

5.5.4 Instructions for prescribing and administering investigational

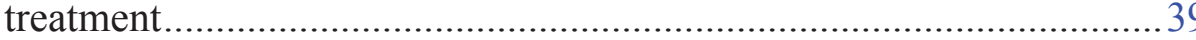

5.5.5 Permitted dose adjustments and interruptions of investigational

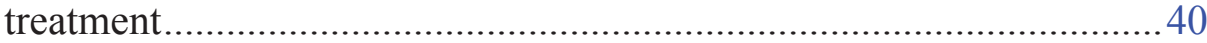

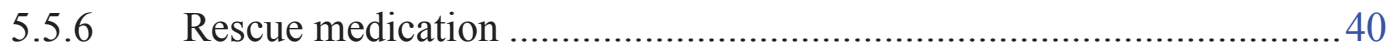

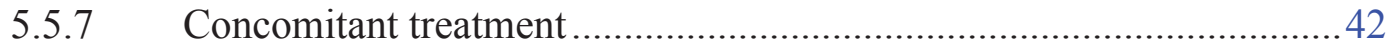

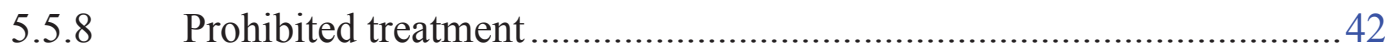

5.5.9 Discontinuation of investigational treatment ......................................4 42

5.5.10 Premature patient withdrawal from the study .................................. 43

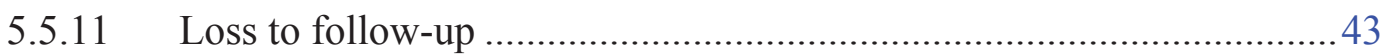

5.5.12 Emergency breaking of assigned treatment code ................................44

5.5.13 Study completion and post-study treatment ......................................4

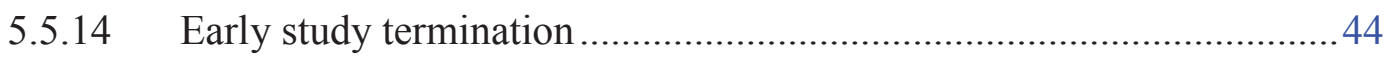

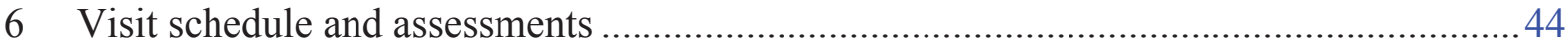

6.1 Information to be collected on screening failures.............................................54

6.2 Patient demographics/other baseline characteristics ..........................................5

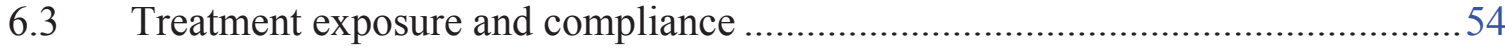

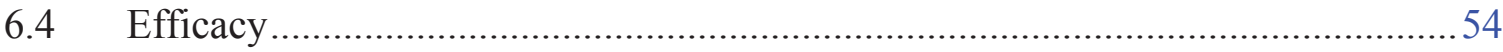

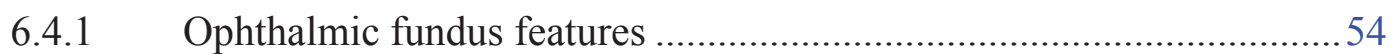

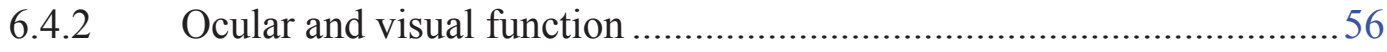

6.4.3 Appropriateness of efficacy assessments ............................................56

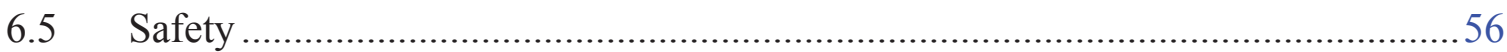

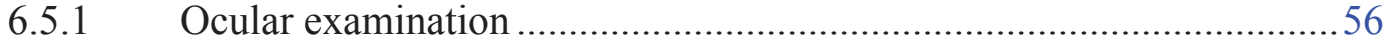

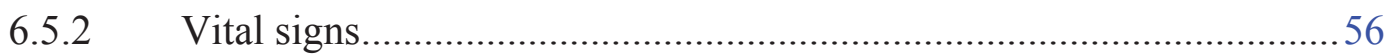

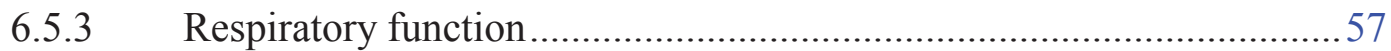

6.5.4 Monitoring of adverse events (ocular and systemic) ..........................57

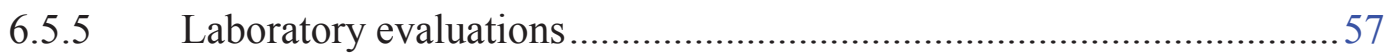

6.5.6 Appropriateness of safety measurements.........................................58

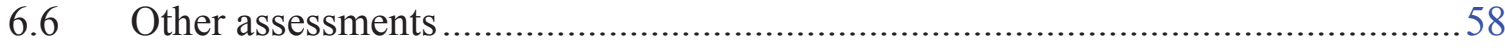

6.6.1 Systemic ranibizumab concentration ...............................................58

6.6.2 Systemic anti-ranibizumab antibody level ......................................5

6.6.3 Systemic vascular endothelial growth factor level..............................59

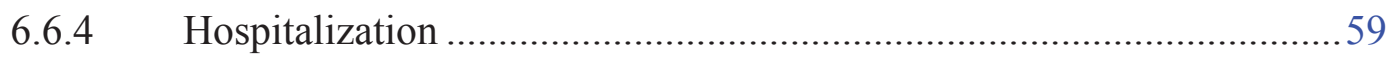

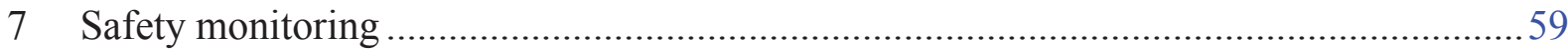

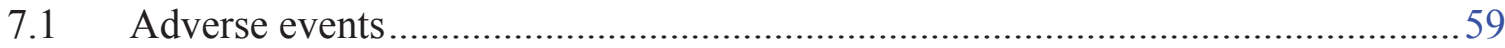

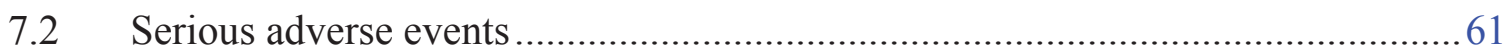




\begin{tabular}{|c|c|}
\hline & rious adverce eyent \\
\hline & \\
\hline
\end{tabular}

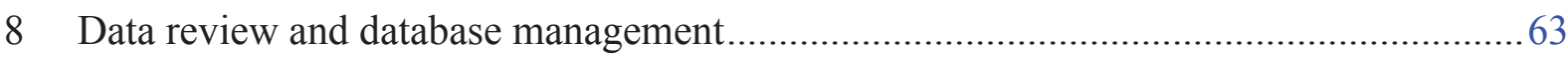

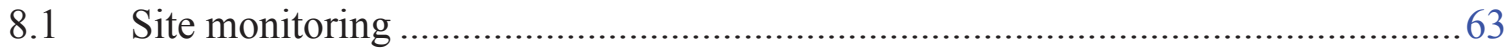

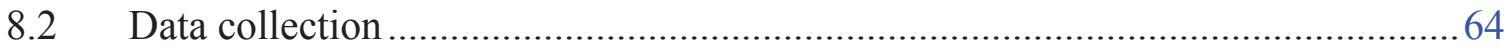

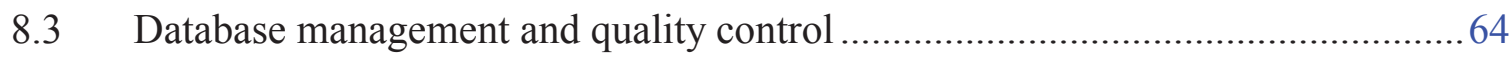

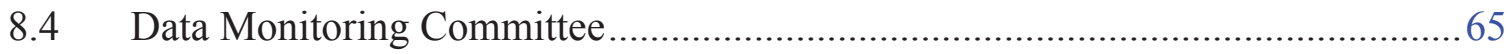

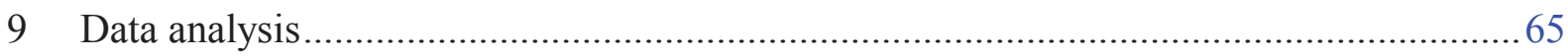

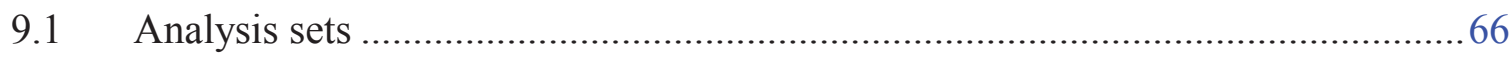

9.2 Patient demographics and other baseline characteristics ....................................66

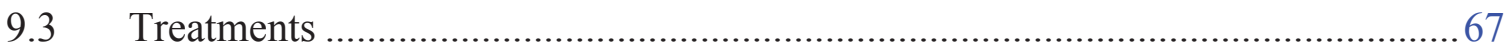

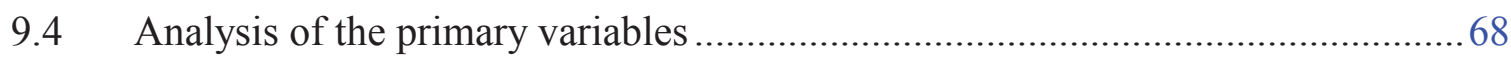

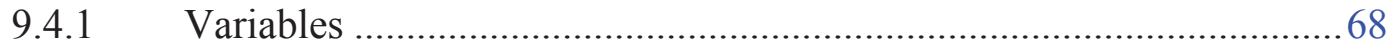

9.4.2 Statistical model, hypothesis, and method of analysis .........................6 68

9.4.3 Handling of missing values/censoring/discontinuations .......................70

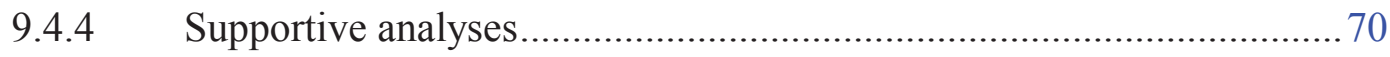

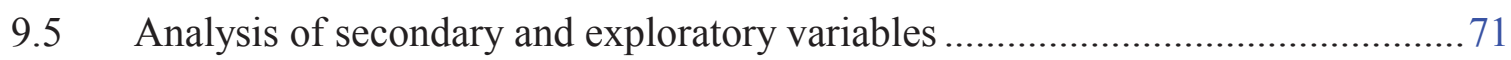

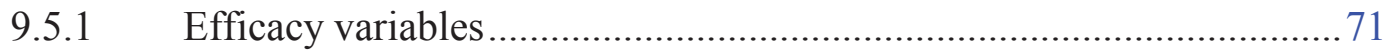

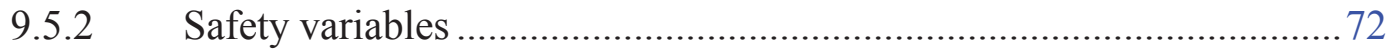

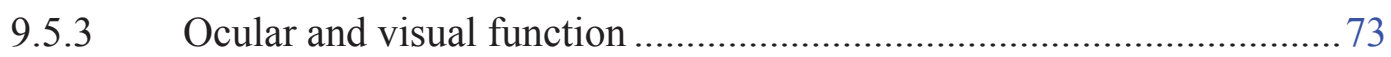

9.5.4 Systemic ranibizumab concentration ............................................. 73

9.5.5 Pharmacogenetics/pharmacogenomics ............................................ 73

9.5.6 Systemic vascular endothelial growth factor level............................. 73

9.5.7 Systemic anti-ranibizumab antibody level .......................................... 74

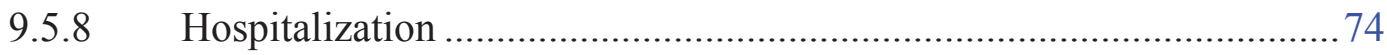

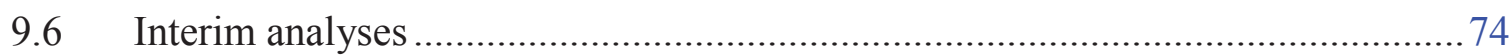

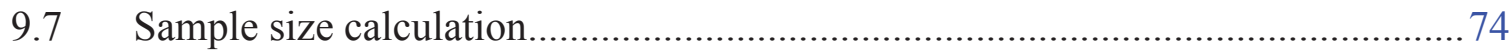

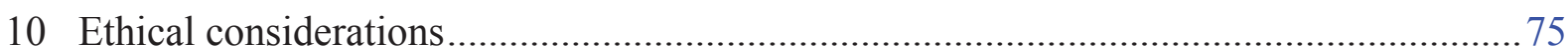

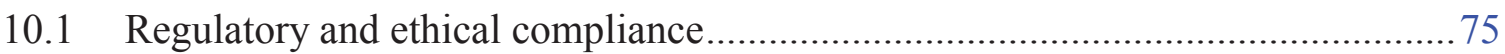

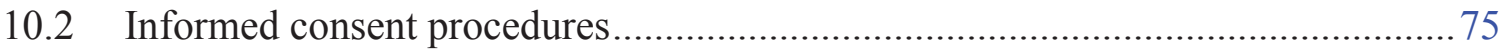

10.3 Responsibilities of the Investigator and IRB/IEC …......................................... 75

10.4 Publication of study protocol and results........................................................ 75

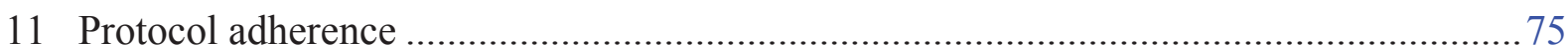

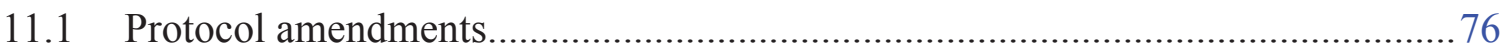

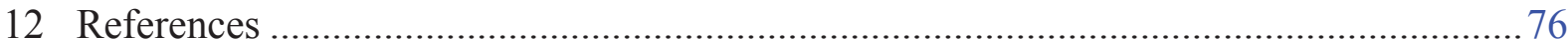


13 Appendix 1: Blood log for systemic ranibizumab / anti-ranibizumab antibody samples collection

14 Appendix 2: Blood log for systemic VEGF samples collection.

\section{List of tables}

Table 5-1

Table 6-1

Table 6-2

Table 6-3

Table 6-4

Table 6-5

Table 6-6

Table 9-1

Table 13-1

Table 14-1

\section{List of figures}

Figure 1-1

Figure 3-1

Figure 3-2

Figure 3-3

Figure 3-4
Prohibited Treatment. 42

Main Assessment Schedule - Screening.............................................46

Main Assessment Schedule - Patients Randomized to Ranibizumab .. 47

Main Assessment Schedule - Patients Randomized to Laser

Therapy

Additional Assessment Schedule - Re-treatment with Ranibizumab...51

Additional Assessment Schedule - Switch to Ranibizumab $0.2 \mathrm{mg}$ Treatment 52

Additional Assessment Schedule - Switch to Laser Therapy. 53

Ranking of Retinopathy of Prematurity Disease

Time schedule for blood sampling for systemic ranibizumab / anti ranibizumab antibody

Time schedule for blood sampling for VEGF 82

Predicted Systemic Exposure After Anti-VEGF Treatment, Classified Based on Toxicological Outcomes........................................2. 24

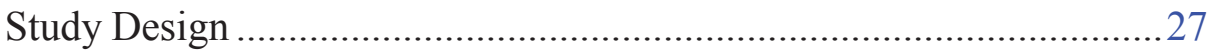

Ocular Exposure Estimated by Area Under the Curve in Vitreous ......29

Systemic Exposure Estimated by Area Under the Curve in Serum ...... 30

Systemic Exposure Estimated by Maximum Concentration in Serum 


\section{List of abbreviations}

AE adverse event

AP-ROP aggressive posterior retinopathy of prematurity

ALP Alkaline phosphatase

ALT Alanine aminotransferase

AST Aspartate transaminase

ATC

Anatomical Therapeutic Chemical (classification system)

AUC

area under the curve

BEAT-ROP Bevacizumab Eliminates the Angiogenic Threat of Retinopathy of Prematurity (study)

BUN Blood urea nitrogen

CFR Code of Federal Regulations

Cmax maximum serum concentration

CTT Clinical Trial Team

DMC Data Monitoring Committee

eCRF electronic case report/record form

EDC electronic data capture

ETROP Early Treatment for Retinopathy of Prematurity (study)

FAS Full analysis set

GCP Good Clinical Practice

$\mathrm{Hb} \quad$ Hemoglobin

IB investigator's brochure

ICH International Conference on Harmonisation of Technical Requirements for Registration of Pharmaceuticals for Human Use

IEC Independent Ethics Committee

IgG immunoglobulin $\mathrm{G}$

IRB Institutional Review Board

IRT Interactive Response Technology

$\mathrm{K}_{\mathrm{D}} \quad$ dissociation constant

MedDRA Medical Dictionary for Regulatory Activities

mRNA messenger ribonucleic acid

OC/RDC Oracle Clinical/Remote Data Capture

PIP Paediatric Investigation Plan 
PK

PLT

PPS

ROP

SAE

SAP

US

VEGF

WHO

WCC pharmacokinetic

Platelet count

Per protocol set

retinopathy of prematurity

serious adverse event

statistical analysis plan

United States of America

vascular endothelial growth factor

World Health Organization

White Cell Count 


\section{Glossary of terms}

\begin{tabular}{|c|c|}
\hline Assessment & A procedure used to generate data required by the study \\
\hline Dose level & The dose of drug given to the patient (total daily or weekly, etc.) \\
\hline Enrollment & $\begin{array}{l}\text { Point/time of patient entry into the study at which informed } \\
\text { consent must be obtained (i.e., prior to starting any of the } \\
\text { procedures described in the protocol) }\end{array}$ \\
\hline Investigational treatment & $\begin{array}{l}\text { All investigational drug(s) whose properties are being tested in the } \\
\text { study as well as their associated treatment controls } \\
\text { This includes any placebos, any active controls, as well as } \\
\text { approved drugs used outside of their indication/approved dosage } \\
\text { or tested in a fixed combination } \\
\text { Investigational treatment generally does not include } \\
\text { protocol specified concomitant background therapies when these } \\
\text { are standard treatments in that indication }\end{array}$ \\
\hline $\begin{array}{l}\text { Investigational treatment } \\
\text { discontinuation }\end{array}$ & $\begin{array}{l}\text { Point/time when patient permanently stops taking } \\
\text { study/investigational treatment for any reason; may or may not } \\
\text { also be the point/time of premature patient withdrawal }\end{array}$ \\
\hline Medication number & $\begin{array}{l}\text { A unique identifier on the label of each investigational/study drug } \\
\text { package in studies that dispense medication using an IRT system }\end{array}$ \\
\hline Patient number & A number assigned to each patient who enrolls into the study \\
\hline Premature withdrawal & $\begin{array}{l}\text { Point/time when the patient exits from the study prior to the } \\
\text { planned completion of all study treatment administration and/or } \\
\text { assessments; at this time all study treatment administration is } \\
\text { discontinued and no further assessments are planned, unless the } \\
\text { patient will be followed for progression and/or survival }\end{array}$ \\
\hline Protocol & $\begin{array}{l}\text { A written account of all the procedures to be followed in a trial, } \\
\text { which describes all the administrative, documentation, analytical } \\
\text { and clinical processes used in the trial }\end{array}$ \\
\hline Randomization number & $\begin{array}{l}\text { A unique identifier assigned to each randomized patient, } \\
\text { corresponding to a specific treatment arm assignment }\end{array}$ \\
\hline Variable & $\begin{array}{l}\text { A measured value or assessed response that is determined in } \\
\text { specific assessments and used in data analysis to evaluate the } \\
\text { drug being tested in the study }\end{array}$ \\
\hline
\end{tabular}




\section{Amendment 2}

\section{Amendment rationale}

The main purpose of this amendment is to:

- Reduce the number from 300 patients planned to be treated in the study to approximately 180 patients planned to be randomized following approval by the European Medicine Agency to modify an agreed Paediatric Investigation Plan (PIP). This PIP modification was justified by the study sponsor in the light of the high unmet medical need, increasing off-label use of anti-VEGF agents in ROP and slow study recruitment rate to ensure earlier availability of the study results. The revised study is considered to provide sufficient statistical power to detect clinically meaningful efficacy results, whilst ensuring that the study be completed in a reasonable timeframe.

In addition, further purpose of this amendment is to:

- Allow results from ophthalmic exams and laboratory evaluations (other than samples collected for determination of ranibizumab or systemic VEGF levels) collected up to two days prior of the screening visit to be used for the study in an effort to minimize the need of repeated procedures to be performed in this vulnerable study population.

In addition, this amendment incorporates changes to the protocol language to improve clarity, accuracy and ensure better adherence to the protocol.

\section{Changes to the protocol}

Major changes are made to the protocol in the following sections:

- Section 4 Population: Information of the study population was changed from 300 patients planned to be treated to approximately 180 patients randomized with corresponding changes to the number of evaluable patients.

- Section 5.5.13 Study completion and post-study treatment: The number of patients was changed from 300 to 180 patients randomized with corresponding changes to the number of evaluable patients. Wording with respect to over recruitment was removed as it is no longer relevant.

- Section 9.4.2 Statistical model, hypothesis, and method of analysis: The primary and key secondary hypotheses testing strategy was revised accordingly due to the reduction in sample size.

- Section 9.7 Sample size calculation: The information was revised due to the reduction in sample size.

Other changes are made to the protocol in the following sections:

- Section 3.6 Risk and benefits: The reference for Zhou et al was updated to now reflect the year of the printed publication instead of the online publication with the respective information added in Section 12. 
- Section 6 Visit schedule and assessments: For Table 6-1 a footnote was added to indicate that examinations performed up to 2 days before the respective visit can also be used. For Table 6-2 and Table 6-3 a footnote was added to indicate that laboratory evaluations which were performed up to 2 days prior to the screening visit can also be used.

- Section 6.3 Treatment exposure and compliance: Clarification was made that for laser therapy, IRT will only be contacted for the first administration (first treatment and rescue) but not for any supplementary laser treatment administration.

- Section 6.4.1 Ophthalmic fundus features: RetCam images taken and results of ophthalmic exams performed up to 2 days before the screening visit can be used. RetCam vendor information was removed.

- Section 6.5.1 Ocular examination: For the screening visit, results from ophthalmic exams performed up to 2 days before this visit can be used.

- Section 6.5.5 Laboratory evaluations: Allow results from laboratory evaluations which were performed up to 2 days prior to the screening visit to be used for the Day 1 visit.

- Section 6.6.3 Systemic vascular endothelial growth factor level: The wording was revised to refer to the Study Operation Manual and Laboratory Manual for information on blood collection.

- Section 8.3 Database management and quality control: Clarification was made between centrally and locally processed laboratory samples and RetCam images.

These changes will be implemented throughout the protocol.

Changes to specific sections of the protocol are shown in the track changes version of the protocol using strike through red font for deletions and red underlined for insertions.

\section{IRBs/IECs}

A copy of this amended protocol will be sent to the Institutional Review Board (IRBs)/Independent Ethics Committee (IECs) and Health Authorities.

The changes described in this amended protocol require IRB/IEC approval prior to implementation.

The changes herein affect the Informed Consent. Sites are required to update and submit for approval a revised Informed Consent that takes into account the changes described in this protocol amendment.

\section{Summary of previous amendments}

\section{Amendment 1 (05-Aug-2015)}

\section{Amendment rationale}

The main purpose of this amendment is to: 
- Include additional safety assessments as requested by Health Authorities and Institutional Review Boards (IRBs)/Independent Ethics Committees during the clinical trial application process. This will enhance the safety monitoring.

- Amend the exclusion criteria to improve clarity. The exclusion criteria \#2 was deleted because it was redundant with e.g. exclusion 11. The exclusion criteria \#11 was amended to clarify that clinically significant comorbidities are exclusionary only if they are assessed by the investigator to have a clinically relevant impact on study participation, any of the study procedures, or on efficacy assessments.

- Better align the rescue procedures and criteria with current clinical practice including changes to the definition of ROP disease that is unchanged, has minimally improved or has worsened.

- Retain patients in the trial for further follow-up in the instances when they are administered with prohibited treatment. This will ensure an appropriate safety monitoring of these patients.

In addition, further purpose of this amendment is to:

- Add nonclinical information requested by Health Authorities (background section).

- Provide information requested by Health Authorities on case reports of ROP infants treated with ranibizumab, in which temporarily reduced serum VEGF levels have been reported (risks and benefits section).

- Remove the efficacy assessment based on the Children's Visual Function Questionnaire as Patient Reported Outcome instrument. This instrument is not age-appropriate for the study population.

- Specify that measures will be taken to keep randomization data and aggregated statistical analysis by treatment confidential from the Clinical Trial Team (CTT) until database lock. Data Management Plan was referenced for additional measures to minimize bias related to treatment knowledge.

- Clarify that, in case RetCam digital photography is not available, the use of indirect ophthalmoscopy is allowed as an alternative. Specify that indirect ophthalmoscopy can be used at the visit following the treatment visit to align with clinical practice.

- Transfer details of the blood sampling route and laser type to the Study Operation Manual.

- Clarify that Central Reading Center data could be analyzed at a later timepoint and delete the related exploratory efficacy variable.

- Specify that odds ratios are used to define superiority.

In addition, this amendment incorporates multiple changes to the protocol language to improve clarity, accuracy and ensure better adherence to the protocol.

At the time of the finalization of this amendment, the study has not yet started recruitment. It is not anticipated that the changes as per this amendment will affect the study population.

\section{Changes to the protocol}

Major changes are made to the protocol in the following sections: 
- Section 6.5 Safety: the monitoring of lower leg length and collection of blood pressure, hematology, clinical chemistry, urinalysis and respiratory function data was added to the safety assessments.

- Section 4.2 Exclusion criteria: the exclusion criteria \#2 was deleted because it was redundant with e.g. exclusion 11. The exclusion criteria \#11 was amended to clarify that clinically significant comorbidities are exclusionary only if they are assessed by the investigator to have a clinically relevant impact on study participation, any of the study procedures, or on efficacy assessments.

- Section 5.5.6 Rescue medication: the definitions for ROP disease that is unchanged, has minimally improved or has worsened were amended to better reflect clinical practice. Criteria to allow the use of ranibizumab $0.2 \mathrm{mg}$ as rescue treatment at Day 8 were added.

- Section 5.5.8 Prohibited treatment: the patient is no longer discontinued from the study if prohibited treatment is administered. Instead, the patient should be discontinued from further investigational treatment but remains in the study for further follow-up.

These changes will be implemented throughout the protocol.

Changes to specific sections of the protocol are shown in the track changes version of the protocol using strike through red font for deletions and red underlined for insertions.

A copy of this amended protocol will be sent to the Institutional Review Board (IRBs)/Independent Ethics Committee (IECs) and Health Authorities.

The changes described in this amended protocol require IRB/IEC approval prior to implementation.

The changes herein affect the Informed Consent. Sites are required to update and submit for approval a revised Informed Consent that takes into account the changes described in this protocol amendment. 


\section{Protocol summary}

\begin{tabular}{|c|c|}
\hline Protocol number & CRFB002H2301 \\
\hline Title & $\begin{array}{l}\text { RAINBOW study: a randomized, controlled study evaluating the efficacy } \\
\text { and safety of RAnibizumab compared with laser therapy for the treatment } \\
\text { of INfants BO} \text { 을 prematurely With retinopathy of prematurity }\end{array}$ \\
\hline Brief title & $\begin{array}{l}\text { RAINBOW study: RAnibizumab compared with laser therapy for the } \\
\text { treatment of INfants BOrn prematurely With retinopathy of prematurity }\end{array}$ \\
\hline $\begin{array}{l}\text { Sponsor and clinical } \\
\text { phase }\end{array}$ & Novartis; phase 3 \\
\hline Investigation type & Drug \\
\hline Study type & Interventional \\
\hline Purpose and rationale & $\begin{array}{l}\text { The purpose of this study is to determine if intravitreal ranibizumab is } \\
\text { superior to laser ablation therapy in the treatment of retinopathy of } \\
\text { prematurity (ROP). The study will assess the ability of these treatments to } \\
\text { lead to regression of active ROP and prevent the development of ocular } \\
\text { complications that are associated with poor visual outcome. } \\
\text { Vascular endothelial growth factor (VEGF) plays an important role in the } \\
\text { pathogenesis of ROP. There is a growing body of evidence supporting } \\
\text { the use of targeted pharmacologic inhibition of VEGF in the management } \\
\text { of ROP. Ranibizumab is a recombinant humanized immunoglobulin G1 } \\
\text { kappa isotype monoclonal antibody fragment targeted against human } \\
\text { VEGF-A; there have been reports of favorable experience with off-label } \\
\text { use of intravitreal anti-VEGF in the management of ROP. }\end{array}$ \\
\hline Primary objective & $\begin{array}{l}\text { To demonstrate that intravitreal ranibizumab } 0.2 \mathrm{mg} \text { has superior efficacy } \\
\text { to laser therapy in the treatment of ROP as measured by the absence of } \\
\text { active ROP and absence of unfavorable structural outcomes in both eyes } \\
24 \text { weeks after starting investigational treatment. } \\
\text { To achieve this outcome, patients cannot fulfill any of the criteria listed in } \\
\text { "Data Analysis" (below). }\end{array}$ \\
\hline Secondary objectives & $\begin{array}{l}\text { Key secondary objectives are: } \\
\text { - To evaluate whether intravitreal ranibizumab } 0.2 \mathrm{mg} \text { has } \\
\text { superior efficacy to intravitreal ranibizumab } 0.1 \mathrm{mg} \text { in the } \\
\text { treatment of ROP as measured by the absence of active ROP } \\
\text { and absence of unfavorable structural outcomes in both eyes } \\
24 \text { weeks after starting investigational treatment } \\
\text { - To evaluate whether intravitreal ranibizumab } 0.1 \mathrm{mg} \text { has } \\
\text { superior efficacy to laser therapy in the treatment of ROP as } \\
\text { measured by the absence of active ROP and absence of } \\
\text { unfavorable structural outcomes in both eyes } 24 \text { weeks after } \\
\text { starting investigational treatment } \\
\text { Other secondary objectives are: } \\
\text { To evaluate the time to intervention with a second modality for } \\
\text { ROP or development of unfavorable structural outcome, or } \\
\text { death }\end{array}$ \\
\hline
\end{tabular}




\begin{tabular}{|c|c|}
\hline & $\begin{array}{l}\text { - To evaluate the recurrence of ROP receiving any post-baseline } \\
\text { intervention at } 24 \text { weeks or before } \\
\text { - To evaluate the ocular and systemic safety of intravitreal } \\
\text { ranibizumab } 0.1 \mathrm{mg} \text { and } 0.2 \mathrm{mg} \text { in the treatment of ROP as } \\
\text { assessed by ocular examination, monitoring of adverse events } \\
\text { (AEs) throughout the study, and by the assessment of length, } \\
\text { weight, head circumference and lower leg length at Baseline, } \\
\text { Day } 85, \text { and Day } 169 \\
\text { - To evaluate the systemic pharmacokinetics of intravitreal } \\
\text { ranibizumab in patients with ROP, as evaluated by sparse } \\
\text { sampling population pharmacokinetic (PK) methods } \\
\text { - To evaluate the effects of investigational treatment on systemic } \\
\text { VEGF levels in patients with ROP, as evaluated by sparse } \\
\text { sampling population concentration-response methods } \\
\text { - To assess the number of ranibizumab administrations needed in } \\
\text { the treatment of patients with ROP }\end{array}$ \\
\hline Exploratory objectives & $\begin{array}{l}\text { - To explore the effects of investigational treatment on the } \\
\text { presence of nystagmus, abnormal fixation behavior, abnormal } \\
\text { pupillary light reaction and refraction } \\
\text { - To explore the effects of investigational treatment on full retinal } \\
\text { vascularization in } 12 \text { clock hours } \\
\text { - To explore the presence of anti-ranibizumab antibodies in } \\
\text { patients with ROP initially treated with ranibizumab } \\
\text { - To explore the efficacy and safety of intravitreal ranibizumab } \\
0.2 \mathrm{mg} \text { administered to patients initially treated with laser } \\
\text { therapy } \\
\text { - To explore the effects of investigational treatment on laboratory } \\
\text { parameters (hematology, clinical chemistry and urinalysis), } \\
\text { blood pressure and requirement for respiratory support }\end{array}$ \\
\hline Study design & $\begin{array}{l}\text { This is a randomized, open-label, } 3 \text {-arm, parallel-group, superiority study } \\
\text { evaluating the efficacy and safety of intravitreal ranibizumab } 0.1 \mathrm{mg} \text {, } \\
\text { intravitreal ranibizumab } 0.2 \mathrm{mg} \text {, and laser therapy for the treatment of } \\
\text { ROP. Patients will receive the assigned investigational treatment on Day } \\
1 \text { to each eye, followed by visits to assess safety and efficacy at regular } \\
\text { intervals during the follow-up epoch. } \\
\text { Patients will be randomized } 1: 1: 1 \text { to } 1 \text { of the following } 3 \text { treatment arms: } \\
\text { - Intravitreal ranibizumab } 0.2 \mathrm{mg} \text { to both eyes on the day of } \\
\text { treatment (Baseline), with up to } 2 \text { re-treatments allowed for each } \\
\text { eye if required } \\
\text { - Intravitreal ranibizumab } 0.1 \mathrm{mg} \text { to both eyes on the day of } \\
\text { treatment (Baseline), with up to } 2 \text { re-treatments allowed for each } \\
\text { eye if required } \\
\text { - Laser ablation therapy to both eyes (at Baseline) } \\
\text { Assessments to address the primary objective will be performed at } \\
\text { Day 169, which is } 24 \text { weeks after starting investigational treatment. }\end{array}$ \\
\hline Population & $\begin{array}{l}\text { The study population will consist of male and female preterm infants with } \\
\text { bilateral ROP who require treatment. Approximately } 180 \text { patients are } \\
\text { planned to be randomized in the study to provide at least } 48 \text { evaluable } \\
\text { patients in each of the } 3 \text { treatment arms (i.e., } 144 \text { evaluable patients in }\end{array}$ \\
\hline
\end{tabular}




\begin{tabular}{|c|c|}
\hline & total). \\
\hline Inclusion criteria & $\begin{array}{l}\text { Patients have to fulfill all of the following criteria prior to receiving the first } \\
\text { investigational treatment: } \\
\text { 1. Signed informed consent from parent(s) or legal guardian(s), in } \\
\text { compliance with local requirements } \\
\text { 2. Male or female preterm infants with a birth weight of less than } \\
1500 \mathrm{~g} \\
\text { 3. Bilateral ROP with } 1 \text { of the following retinal findings in each eye: } \\
\text { - Zone I, stage } 1+, 2+, 3 \text { or } 3+\text { disease, or } \\
\text { - Zone II, stage } 3+\text { disease, or } \\
\text { - Aggressive posterior ROP }\end{array}$ \\
\hline Exclusion criteria & $\begin{array}{l}\text { Patients fulfilling any of the following criteria prior to receiving the first } \\
\text { investigational treatment are not eligible for inclusion in this study. } \\
\text { Investigational treatment is not clinically appropriate for the following } \\
\text { patients: } \\
\text { 1. Have ROP disease characteristic in either eye, other than that } \\
\text { listed in "Inclusion Criteria" above, at the time of the first } \\
\text { investigational treatment } \\
\text { 2. Have a history (either the patient or the mother) of } \\
\text { hypersensitivity to any of the investigational treatments or to } \\
\text { drugs of similar chemical classes } \\
\text { Risk of confounding efficacy and/or safety assessments in the following } \\
\text { patients: } \\
\text { 3. Have received any previous surgical or nonsurgical treatment for } \\
\text { ROP (e.g., ablative laser therapy or cryotherapy, vitrectomy) } \\
\text { 4. Have been previously exposed to any intravitreal or systemic } \\
\text { anti-VEGF agent (either the patient or the mother during this } \\
\text { child's pregnancy) } \\
\text { 5. Have used (either the patient or the mother) other investigational } \\
\text { drugs as part of another clinical study (other than vitamins and } \\
\text { minerals) within } 30 \text { days or within } 5 \text { half-lives of the other } \\
\text { investigational drug, whichever is longer } \\
\text { 6. Have ocular structural abnormalities that are assessed by the } \\
\text { Investigator to have a clinically significant impact on study } \\
\text { assessments } \\
\text { 7. Have active ocular infection within } 5 \text { days before or on the day of } \\
\text { first investigational treatment } \\
\text { 8. Have a history of hydrocephalus requiring treatment } \\
\text { 9. Have a history of any other neurological conditions that are } \\
\text { assessed by the Investigator to have a significant risk of severe } \\
\text { impact on visual function } \\
\text { 10. Have any other medical conditions or clinical significant } \\
\text { pomorbidities or personal circumstances that are assessed by } \\
\text { adequately dilated, unable to comply with the visit schedule) }\end{array}$ \\
\hline $\begin{array}{l}\text { Investigational and } \\
\text { reference therapy }\end{array}$ & $\begin{array}{l}\text { Ranibizumab } 10 \mathrm{mg} / \mathrm{mL} \text { solution for injection (labeled as } \\
\text { "RFB002 } 0.5 \mathrm{mg} / 0.05 \mathrm{~mL} \text { ") } \text { supplied in glass vials for single use by }\end{array}$ \\
\hline
\end{tabular}




\begin{tabular}{|c|c|}
\hline & $\begin{array}{l}\text { Novartis Drug Supply Management. The storage conditions will be } \\
\text { described on the medication label. } \\
\text { Conventional laser ablative therapy }\end{array}$ \\
\hline Efficacy assessments & $\begin{array}{l}\text { Ophthalmic fundus features } \\
\text { These will be assessed in both eyes using images taken by RetCam } \\
\text { digital photography and/or as assessed by indirect ophthalmoscopy: } \\
\text { - Features of active ROP disease: } \\
\text { - ROP disease - zone, stage, extent by clock hours, and } \\
\text { characteristics of vascular changes of plus disease (extent } \\
\text { by quadrants and severity) } \\
\text { - Extra-retinal vessels extending from the retina into the } \\
\text { vitreous and judged to be a sign of active ROP disease } \\
\text { - Features of late sequelae of ROP } \\
\text { - Substantial temporal retinal vessel dragging causing } \\
\text { - Rbnormal structural features/ macular ectopia } \\
\text { pole } \\
\text { - Posterior retinal fold involving the macula } \\
\text { - Retinal detachment involving the macula } \\
\text { - Retinal detachment not involving the macula } \\
\text { - Degree of full retinal vascularization } \\
\text { Ocular and visual function } \\
\text { - Presence of nystagmus } \\
\text { - Abnormal fixation behavior } \\
\text { - Abnormal pupillary light reaction } \\
\text { - Refraction (in spherical equivalence) as assessed by } \\
\text { retinoscopy or auto-refraction after cycloplegia }\end{array}$ \\
\hline Safety assessments & $\begin{array}{l}\text { - } \quad \text { Ocular examination } \\
\text { - } \quad \text { Blood pressure } \\
\text { - } \quad \text { Length, weight, head circumference and lower leg length } \\
\text { - } \quad \text { Adverse event monitoring (ocular and systemic) } \\
\text { - Laboratory parameters (hematology, clinical chemistry and urinalysis) } \\
\text { - } \quad \text { Requirement for respiratory support }\end{array}$ \\
\hline Other assessments & $\begin{array}{l}\text { - Assessment of systemic ranibizumab concentration (in patients who } \\
\text { receive initial ranibizumab treatment and with an odd patient } \\
\text { identification number) } \\
\text { - Assessment of systemic VEGF levels (in patients who receive initial } \\
\text { ranibizumab treatment and with an even patient identification number } \\
\text { as well as patients who receive initial laser therapy) } \\
\text { - Assessment of systemic anti-ranibizumab antibody levels (in patients } \\
\text { in whom systemic ranibizumab concentration is assessed, provided } \\
\text { sufficient serum sample volume) } \\
\text { - Duration of hospitalization and weight at discharge home }\end{array}$ \\
\hline Data analysis & $\begin{array}{l}\text { The Full Analysis Set analyzed according to intent-to-treat principles will } \\
\text { be used to assess efficacy. }\end{array}$ \\
\hline
\end{tabular}


The primary efficacy variable is the absence of active ROP and absence of unfavorable structural outcomes in both eyes 24 weeks after starting investigational treatment, as assessed by the Investigator. To achieve this outcome, patients cannot fulfill any of the following criteria:

- Death at or before the 24-week assessment visit

- Requires intervention for ROP in either eye at or before the 24week assessment visit with a treatment modality other than the modality of the first investigational treatment

- Have active ROP in either eye at the 24-week assessment visit as defined by any of the following features:

- Vessel dilatation of plus disease in at least 2 quadrants of the eye (some persisting tortuosity is allowed)

- Extra-retinal vessels extending from the retina into the vitreous and judged to be a sign of active ROP disease

- Have unfavorable structural outcomes in either eye at or before the 24-week assessment visit as defined by the presence of any of the following features:

- Retrolental membrane obscuring the view of the posterior pole

- Substantial temporal retinal vessel dragging causing abnormal structural features/ macular ectopia

- Posterior retinal fold involving the macula

- Retinal detachment involving the macula

A 2-sided family-wise type I error rate of $5 \%$ will be used. Two-sided exact $95 \%$ confidence intervals will also be presented for the primary variable event rate within each treatment arm.

Secondary and exploratory variables are described in Section 9.5.1.

\section{Statistical model, hypothesis, and method of analysis}

A three-step sequential testing procedure will be used for primary (ranibizumab $0.2 \mathrm{mg}$ against laser) and two key secondary comparisons (ranibizumab $0.1 \mathrm{mg}$ against laser and ranibizumab $0.2 \mathrm{mg}$ against $0.1 \mathrm{mg}$ ). If the efficacy comparison at any step is not statistically significant, the remaining efficacy comparisons will be assessed descriptively. Otherwise the comparison will continue to the next step.

All hypotheses will be tested at a pre-specified level of significance (two sided $\alpha=0.05)$. This testing procedure controls familywise type I error rate at a pre-specified level of significance. Sequential steps are given in the followings:

Step 1: (primary comparison)

$\mathrm{H}_{01}: \pi$ Ranibizumab $0.2 \mathrm{mg}-\pi$ Laser $=0$

versus

$\mathrm{H}_{\text {A1 }}$ : $\pi$ Ranibizumab $0.2 \mathrm{mg}-\pi$ Laser $\neq 0$

where $\pi$ Treatment arm is the unknown proportion of patients with absence of active ROP and absence of unfavorable structural outcomes in both eyes 24 weeks after starting investigational treatment, as assessed by the Investigator in the relevant treatment arm. 


\begin{tabular}{|c|c|}
\hline & 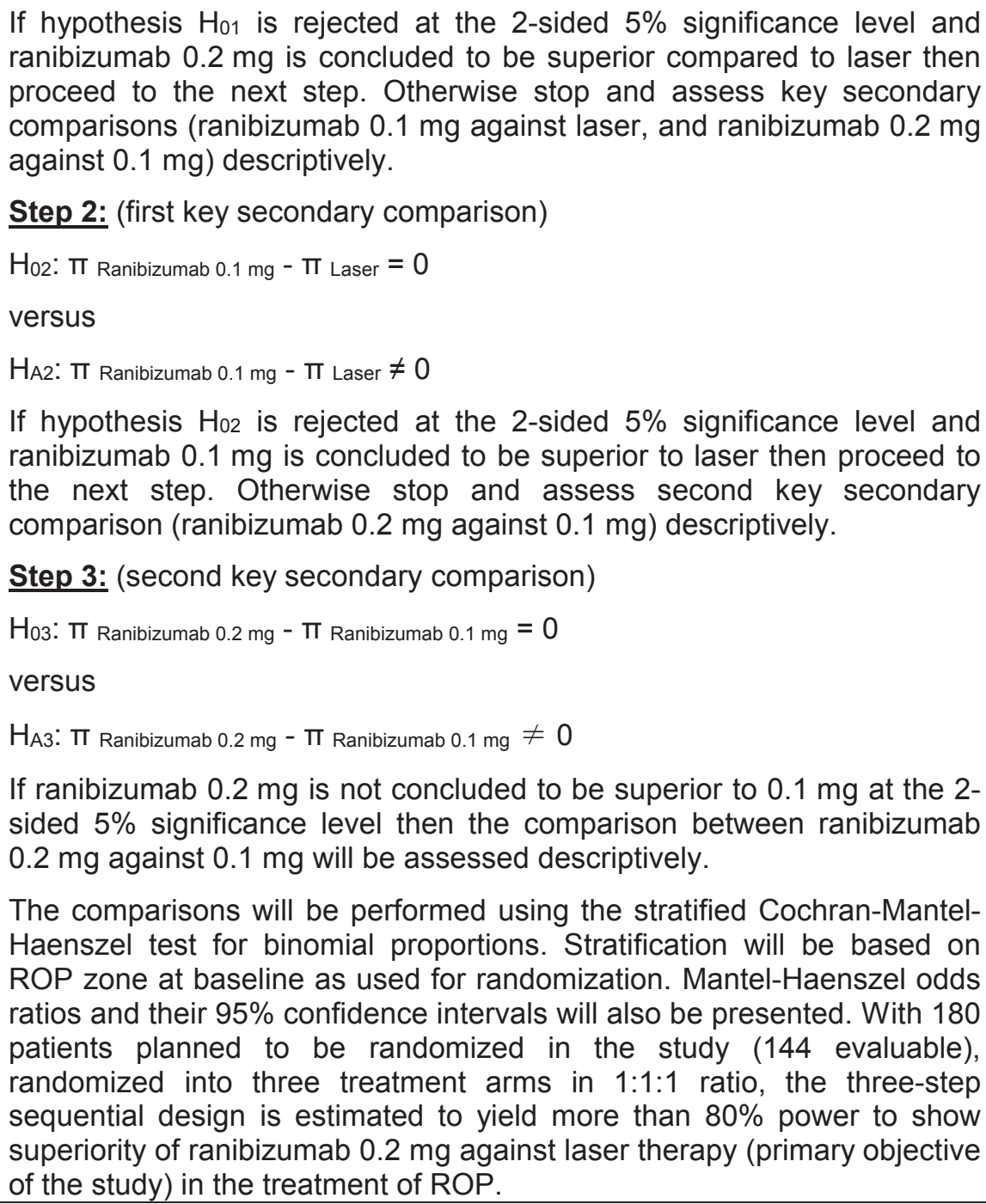 \\
\hline Key words & $\begin{array}{l}\text { Randomized, open-label, controlled study; intravitreal ranibizumab; laser } \\
\text { ablation therapy; retinopathy of prematurity; preterm infants; RAINBOW }\end{array}$ \\
\hline
\end{tabular}




\section{$1.1 \quad$ Background}

Retinopathy of prematurity (ROP) is a vasoproliferative pathologic process that occurs in the incompletely vascularized, developing retina of low birth-weight preterm neonates. Incidence and severity of ROP were found to rise with degree of prematurity at birth, low gestational age and low birth-weight being the main risk factors (Hartnett and Penn 2012, Fierson et al 2013). The vascular changes of ROP may be mild and regress completely with time without major long-term sequelae, or may increase in severity and lead to macular dragging, total retinal detachment, severe visual impairment, and lifelong blindness (Hardy et al 2004). Retinopathy of prematurity is a significant cause of blindness in children in both developed and developing countries with approximately 50000 children blind from ROP worldwide (Gilbert 2008, Mintz-Hittner et al 2011).

Ablation of the peripheral avascular retina with cryotherapy was the first standard treatment for ROP, with efficacy demonstrated in the CRYO-ROP study (Cryotherapy for Retinopathy of Prematurity Cooperative Group 1988). At 15 years after cryotherapy, treated eyes had 40\% less unfavorable structural outcomes and 30\% less unfavorable visual acuity outcomes, as compared with the control eyes (Palmer et al 2005). In the 1990s, the use of laser photocoagulation to ablate the peripheral avascular retina gained widespread acceptance, and this modality has now almost completely replaced cryotherapy. Laser ablation therapy is associated with better long-term structural, visual, and refractive outcomes than cryotherapy (Connolly et al 2002, Ng et al 2002, Houston et al 2013).

In 2003, the Early Treatment for Retinopathy of Prematurity (ETROP) study demonstrated that early ablation therapy was associated with better visual and structural outcomes at 9 months, compared to when therapy was initiated at later stages of ROP (Good 2004). The authors developed a clinical algorithm to identify for early treatment those eyes with ROP characteristics that had the highest risk for retinal detachment and blindness (Type I ROP), while minimizing treatment of those eyes with ROP characteristics likely to show spontaneous regression (Type II ROP). The results suggested that, in most circumstances, peripheral retinal ablation should be considered for any eye with Type I ROP:

- Zone I, any stage ROP with plus disease

- Zone I, stage 3 ROP with or without plus disease

- Zone II, stage 2 or 3 ROP with plus disease

Conversely, continued serial examinations should be considered for any eye with Type II ROP:

- Zone I, stage 1 or 2 ROP without plus disease

- Zone II, stage 3 ROP without plus disease

Treatment should be considered for any eye with Type II ROP if it progresses to Type I status (Good 2004). 
The findings from the ETROP study, incorporating revisions to the ROP classification system, provided the basis for current clinical guidelines recommending that the following types of ROP should be treated (International Committee for the Classification of Retinopathy of Prematurity 2005, Royal College of Paediatrics and Child Health 2008, Fierson et al 2013):

- Zone I, any ROP with plus disease

- Zone I, stage 3 ROP without plus disease

- Zone II, stage 3 ROP with plus disease

- Aggressive posterior ROP (AP-ROP)

With respect to zone II stage $2+$ ROP, there are conflicting opinions on whether the available clinical data support the benefits of early treatment (Royal College of Paediatrics and Child Health 2008). Hence, the treatment guidelines for this ROP category differ on whether treatment should be initiated (Fierson et al 2013) or allow the decision to treat or monitor closely to be made by an experienced ophthalmologist (Royal College of Paediatrics and Child Health 2008).

Despite improvement in the technology and timing of ROP treatment, it remains a leading cause of childhood blindness worldwide. The ETROP study reported $14 \%$ unfavorable visual acuity outcome and $9 \%$ unfavorable structural outcome at 9 months despite ablation therapy (Good 2004). In addition, laser ablation therapy may not be possible for certain patients. The conduct of laser therapy usually requires general anesthesia and adequate visualization of the retina, which may be problematic in preterm neonates with associated comorbidities.

Both cryotherapy and laser therapy ablate the avascular peripheral retina and destroy the majority of the retinal cells that produce vascular endothelial growth factor (VEGF), which plays an important role in the pathogenesis of ROP (Smith 2008). Because of the role of VEGF in ROP, there is a growing body of evidence supporting the use of targeted pharmacologic inhibition of VEGF in the management of ROP (Sonmez et al 2008, Sato et al 2009). There are a number of reports of favorable experience with the off-label use of intravitreal bevacizumab or ranibizumab in the management of ROP (Chung et al 2007, Travassos et al 2007, Honda et al 2008, Kusaka et al 2008, Lalwani et al 2008, Mintz-Hittner and Kuffel Jr 2008, Quiroz-Mercado et al 2008, Ahmed et al 2010, Altinsoy et al 2010, Dorta and Kychenthal 2010, Law et al 2010, Lee et al 2010, Nazari et al 2010, Zepeda-Romero et al 2010, Harder et al 2011, Mintz-Hittner et al 2011, Wu et al 2011, Lin et al 2012, Mota et al 2012, Castellanos et al 2013, Kim et al 2014). Most reports used approximately $50 \%$ of the adult intravitreal dose per eye, although efficacy has also been reported with lower doses (Harder et al 2011, Kim et al 2014).

In a prospective, multicenter, randomized clinical study, intravitreal bevacizumab was found to be more efficacious than conventional laser ablation therapy among infants with stage 3+ ROP (Bevacizumab Eliminates the Angiogenic Threat of ROP (BEAT-ROP) study) (Mintz-Hittner et al 2011). The primary outcome of the study was the recurrence of retinal neovascularization before 54 weeks postmenstrual age that required re-treatment. It was found that among the subgroup of infants with zone I disease the recurrence rate was $6 \%$ with bevacizumab and $42 \%$ with conventional laser therapy. Moreover, bevacizumab therapy resulted in a mild anatomical retinal abnormality in only 1 eye of 31 infants, whereas conventional laser treatment resulted in a mild anatomical abnormality in 16 eyes, and a 
severe abnormality in 2 eyes, of 33 infants. Although the differences in outcomes were not statistically significant among infants with posterior zone II ROP, the results suggested a similar efficacy trend. An interesting finding of the BEAT-ROP study was that treatment with bevacizumab appeared to allow for continued vessel growth into the peripheral retina. In contrast, ablation therapy destroyed large areas of the peripheral retina and may affect associated vascular growth. If anti-VEGF agents allow more normal retinal vascularization to occur, it is anticipated that the rate of abnormal visual acuity, restricted visual fields, refractive errors, amblyopia, and strabismus may be reduced. This was demonstrated when patients from the BEAT-ROP study were assessed at a mean age of 2.5 years, with more myopia found in eyes that received laser treatment than in eyes that received intravitreal bevacizumab (Geloneck et al 2014).

A number of limitations have been noted in the BEAT-ROP study (Darlow et al 2013). The stage $3+$ patients who were studied represent only a subset of the types of ROP disease that require treatment; a relatively high dose of anti-VEGF was evaluated; a lack of assessment of systemic bevacizumab and VEGF levels; and an unexpectedly high failure rate observed in the control laser therapy arm. Therefore, despite reports of potential benefits of anti-VEGF in the treatment of ROP, a number of questions remain unanswered. There is concern in the medical community that preterm neonates with ROP are treated with anti-VEGF agents with insufficient evidence of clinical efficacy compared to standard of care therapy, lack of understanding of the optimal dose to be used, as well as information on the long-term safety impact of intravitreal anti-VEGF use in a developing infant (Lee et al 2011, Darlow et al 2013). This is especially the case with bevacizumab, which is not approved for intravitreal use.

\subsubsection{Ranibizumab}

Ranibizumab is a recombinant humanized immunoglobulin G1 kappa isotype monoclonal antibody fragment targeted against human VEGF-A. It binds with high affinity to VEGF-A isoforms generated by alternative messenger ribonucleic acid (mRNA) splicing, such as VEGF121, VEGF165, and their biologically active proteolytic cleavage product VEGF110.

\section{Physical, Chemical, and Pharmaceutical Properties and Formulation}

Detailed information on the physical, chemical, and pharmaceutical properties of ranibizumab is described in the investigator's brochure (IB).

Ranibizumab is available as a $10 \mathrm{mg} / \mathrm{mL}$ solution for intravitreal injection. Novartis is the marketing authorization holder for Lucentis ${ }^{\circledR} 10 \mathrm{mg} / \mathrm{mL}$ solution for injection in the European Union (EMEA/H/C/000715) and worldwide with the exception of the United States of America (US). The $10 \mathrm{mg} / \mathrm{mL}$ solution for injection is approved and commercialized globally.

The formulation is presented as a single-use sterile, clear to slightly opalescent, colorless to pale yellow and preservative free aqueous solution for intravitreal injection supplied in glass vials. All primary packaging materials are standard quality, suitable for packaging sterile liquid products, and comply with relevant pharmacopoeial requirements.

A syringe suitable to inject volumes of $10 \mu \mathrm{L}$ and $20 \mu \mathrm{L}$ of the solution into the eye will be used. This syringe will be a sterile, single-use disposable syringe intended for medical 
purposes. The syringe will be individually packaged. The graduations will be spaced such that low volumes in the range of several $\mu \mathrm{L}$ can be delivered accurately. A standard injection needle (e.g., $30 \mathrm{G}, 1 / 2$ inch stainless steel) as for the adults will be used for the injection in premature neonates.

\section{Neonatal Nonclinical and Clinical Information}

The IB contains information on nonclinical studies of ranibizumab, as well as clinical studies in age-related macular degeneration, diabetic macular edema, retinal vein occlusion, and pathologic myopia. Additional information relevant to the neonatal use of ranibizumab is described below.

Vascular endothelial growth factor plays an important angiogenic role in the embryo/fetus, during the postnatal period and in the adult life.

The 3 preclinical studies described below involved more extreme inhibition of systemic VEGF (e.g. transgenic mice and high dose of systemically administered VEGFR-1 decoy construct and VEGF-A antibody) than seen following intravitreal administration of a VEGF inhibitor.

Gerber et al 1999 reported on a conditional VEGF-knock out mouse model; incomplete/partial suppression of VEGF beginning at approximately Day 4 after birth was lethal to $38 \%$ of mice by Day 7. In surviving animals there was reported growth delays and liver maturation was delayed. Other organs evaluated (heart, kidney, lung and spleen) appeared small but normal. By Day 27 after birth, 10\% of mice recovered body weight compared to wild type control mice.

Gerber et al. 1999 also dosed healthy newborn mice with either a mouse VEGFR-1 decoy construct (to deplete VEGF-A and VEGF-B), or an isotypic control protein. Weight loss was seen at doses as low as $1 \mathrm{mg} / \mathrm{kg}$. The highest dose tested, $25 \mathrm{mg} / \mathrm{kg}$, was lethal (deaths within 4-6 days of treatment). Target tissues identified were the fat stores (depleted), kidney (hemorrhage and altered growth), delayed growth of the lung and liver; and single-cell necrosis in the liver, heart, pancreas and spinal ganglia.

Malik et al. 2006 compared the effects of an anti-VEGF-A antibody and the mouse VEGFR-1 decoy construct in newborn mice at maximal pharmacodynamics doses. Both biologics decreased survival and weight-gain in the mice. Treatment delayed growth and vascularization of the liver, spleen, lung, heart, kidneys, and thymus.

Nonclinical toxicology data (ocular and systemic) on intravitreal administered ranibizumab were generated in cynomolgus monkeys that were 2 to 3.5 years old at study start, which is equivalent to adolescents/adults in terms of ocular and systemic organ development stage (Boothe et al 1985, Kiely et al 1987, Edward and Kaufman 2003, Qiao-Grider et al 2007). In monkeys, intravitreal administration of ranibizumab elicited essentially dose-dependent intraocular inflammatory responses that, at least in part, were considered to be an immunemediated antibody response to a humanized protein, and thus of questionable relevance to humans. No systemic adverse effects were recorded in nonhuman primates administered up to $2 \mathrm{mg} /$ eye for 26 weeks or when the intravitreal doses given to monkeys resulted in levels of 
systemically circulating ranibizumab that were higher than those observed in the clinical situation.

In pregnant cynomolgus monkeys, intravitreal administration of ranibizumab did not elicit developmental toxicity/teratogenicity and had no effect on weight/structure of the placenta. Bevacizumab or aflibercept administered intravenously to pregnant animals cause skeletal effects to the embryo/fetus (EMEA 2006, EMEA 2013). Therefore, the absence of embryo-fetal toxicity with ranibizumab is attributed to the very low systemic exposures after intravitreal administration and, potentially, from the inability of the antigen-binding fragment to cross the placental barrier due to lack of a crystallizable fragment region (unless antiranibizumab antibodies form and act as carrier proteins, thereby enabling placental transfer via binding to the neonatal crystallizable fragment receptor). However, due to its pharmacological mode of action, ranibizumab must be regarded as potentially teratogenic and embryo-fetal toxic.

Novartis did not conduct toxicology studies of ranibizumab administered intravitreally in premature/neonate monkeys for ethical reasons, i.e., these studies would not have generated new safety information compared to those generated from systemic toxicity studies of other VEGF inhibitors administered intravenously or intraperitoneally in rodents (genetically modified mice, mice and rats), nonhuman primates and humans. In these systemic toxicity studies, the brain, liver, skeletal and urinary systems were identified as potential target organ of toxicity in newborn and adults (Kitamoto et al 1997, Gerber et al 1999, Ryan et al 1999, Jin et al 2002, Eremina et al 2006, Eremina et al 2008, De Pasquale et al 2011). In particular, a phase 1 dose escalation study on the use of bevacizumab in young patients (1-20 years old) with refractory solid tumors identified proteinuria as a non dose limiting side effect (Glade Bender et al 2008). One case of metaphyseal bone lesions (resolved 2 months after dosing) was also reported in a 4.5 -month-old child administered $10-\mathrm{mg} / \mathrm{kg}$ bevacizumab (Smith et al 2008).

The highest dose/volume $(0.2 \mathrm{mg} / 20 \mu \mathrm{L})$ of ranibizumab which will be evaluated in the ROP clinical trial in premature neonates is predicted to have much lower systemic exposure (more than 10-fold lower serum pharmacokinetic (PK) area under the curve (AUC) normalized based on affinity to VEGF) than the above preclinical and clinical studies of anti-VEGF treatment that reported toxicological events (Figure 1-1). Exposure for ranibizumab, bevacizumab, and the soluble VEGF receptor 1 linked to IgG was calculated using 1-compartment and 2-compartment PK models, respectively. The PK models were scaled by body weight of humans of different ages and animals. Exposure was calculated as AUC for dose regimens and over a time interval reported in the cited studies. Concentration of ranibizumab and bevacizumab/ soluble VEGF receptor 1-IgG was normalized by affinity to VEGF of these drugs so that exposure is compared on the same scale. This modeling of anticipated systemic exposures to ranibizumab in the ROP population, at the doses proposed of $0.1 \mathrm{mg}$ and $0.2 \mathrm{mg}$ outlined above, estimates that the VEGF affinity normalized serum PK exposure is approximately $1.5 \mathrm{logs}$ below the exposures of bevacizumab that has resulted in adverse kidney and bone effects. 


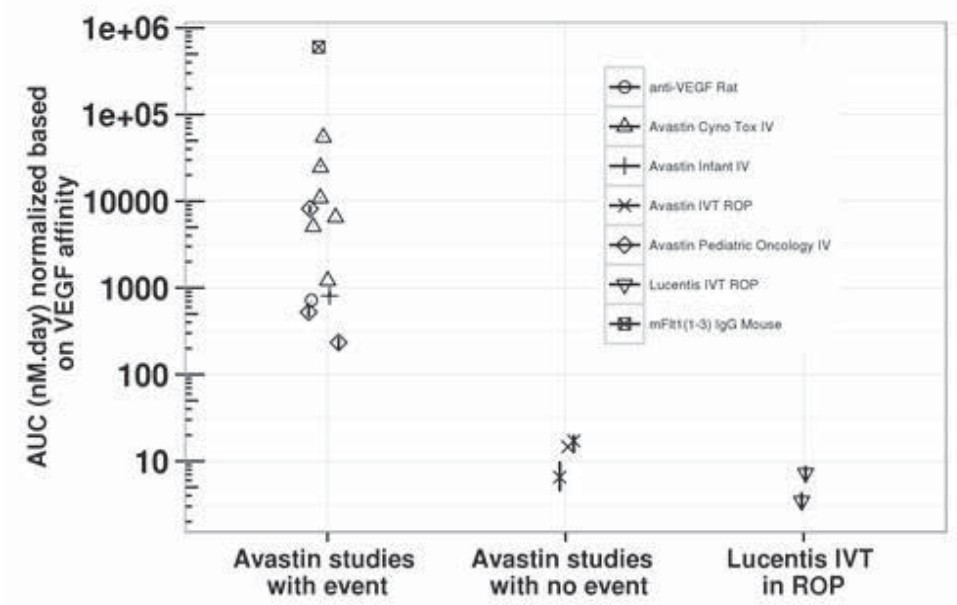

Abbreviations: AUC - area under the curve; IgG, immunoglobulin G; IV - intravenous; IVT - intravitreal; ROP, retinopathy of prematurity; VEGF - vascular endolethial growth factor

The dosing regimen proposed in the ROP clinical trial is also comparable to the one currently used by physicians treating ROP (age $\geq 26$ weeks) with unlicensed anti-VEGF agents such as bevacizumab. In these studies, laser therapy performed after bevacizumab administration has been reported to increase systemic drug exposures by damaging the natural barrier that represents a full-thickness retina (Wu et al 2011). However, no systemic complication (cardiovascular, respiratory, neurologic or renal) attributable to bevacizumab treatment (0.40-1.25 mg/eye) has been recorded so far in premature neonates (Micieli et al 2009, Law et al 2010, Sahin et al 2013, Wu et al 2013).

\subsection{Purpose}

The purpose of this study is to determine if intravitreal ranibizumab is superior to laser ablation therapy in the treatment of ROP. The study will assess the ability of these treatments to lead to regression of active ROP and prevent the development of ocular complications that are associated with poor visual outcome.

This study is part of the European Medicines Agency Paediatric Investigation Plan for ranibizumab (Lucentis).

\section{$2 \quad$ Study objectives}

\subsection{Primary objective}

To demonstrate that intravitreal ranibizumab $0.2 \mathrm{mg}$ has superior efficacy to laser therapy in the treatment of ROP.

The primary efficacy variable is the absence of active ROP and absence of unfavorable structural outcomes in both eyes 24 weeks after starting investigational treatment, as assessed 
by the Investigator. To achieve this outcome, patients cannot fulfill any of the following criteria:

- Death at or before the 24-week assessment visit

- Requires intervention for ROP at or before the 24-week assessment visit with a treatment modality other than the modality of the first investigational treatment

- Have active ROP in either eye at the 24-week assessment visit as defined by the presence of any of the following features:

- Vessel dilatation of plus disease in at least 2 quadrants (some persisting tortuosity is allowed)

- Extra-retinal vessels extending from the retina into the vitreous and judged to be a sign of active ROP disease

- Have unfavorable structural outcomes in either eye at or before 24-week assessment visit as defined by the presence of any of the following features:

- Retrolental membrane obscuring the view of the posterior pole

- Substantial temporal retinal vessel dragging causing abnormal structural features/ macular ectopia

- Posterior retinal fold involving the macula

- Retinal detachment involving the macula

\subsection{Secondary objectives}

Key secondary objectives are:

- To evaluate whether intravitreal ranibizumab $0.2 \mathrm{mg}$ has superior efficacy to intravitreal ranibizumab $0.1 \mathrm{mg}$ in the treatment of ROP as measured by the absence of active ROP and absence of unfavorable structural outcomes in both eyes 24 weeks after starting investigational treatment, as assessed by the Investigator

- To evaluate whether intravitreal ranibizumab $0.1 \mathrm{mg}$ has superior efficacy to laser therapy in the treatment of ROP as measured by the absence of active ROP and absence of unfavorable structural outcomes in both eyes 24 weeks after starting investigational treatment, as assessed by the Investigator

Other secondary objectives are:

- To evaluate the time to intervention with a second modality for ROP or development of unfavorable structural outcome or death

- To evaluate the recurrence of ROP receiving any post-baseline intervention at 24 weeks or before

- To evaluate the ocular and systemic safety of intravitreal ranibizumab $0.1 \mathrm{mg}$ and $0.2 \mathrm{mg}$ in the treatment of ROP as assessed by ocular examination, monitoring of AEs throughout the study, and by the assessment of length, weight, head circumference and lower leg length at Baseline, Day 85, and Day 169

- To evaluate the systemic pharmacokinetics of intravitreal ranibizumab in patients with ROP, as evaluated by sparse-sampling population PK methods 
- To evaluate the effects of investigational treatment on systemic VEGF levels in patients with ROP, as evaluated by sparse-sampling population concentration-response methods

- To assess the number of ranibizumab administrations needed in the treatment of patients with ROP

\subsection{Exploratory objectives}

- To explore the effects of investigational treatment on the presence of nystagmus, abnormal fixation behavior, abnormal pupillary light reaction and refraction

- To explore the effects of investigational treatment on full retinal vascularization in 12 clock hours

- To explore the presence of anti-ranibizumab antibodies in patients with ROP initially treated with ranibizumab

- To explore the efficacy and safety of intravitreal ranibizumab $0.2 \mathrm{mg}$ administered to patients initially treated with laser therapy

- To explore the effects of investigational treatment on laboratory parameters (hematology, clinical chemistry and urinalysis), blood pressure and requirement for respiratory support

\section{$3 \quad$ Investigational plan}

\subsection{Study design}

This is a randomized, open-label, 3-arm, parallel-group, superiority study evaluating the efficacy and safety of intravitreal ranibizumab $0.1 \mathrm{mg}$, intravitreal ranibizumab $0.2 \mathrm{mg}$, and laser therapy for the treatment of ROP (Figure 3-1). Screening and randomization can occur up to 3 days before the administration of the first investigational treatment (Section 6). Eligible patients will be those with bilateral ROP with 1 of the following retinal findings in each eye:

- Zone I, stage $1+, 2+, 3$ or $3+$ disease, or

- Zone II, stage 3+ disease, or

- AP-ROP

Patients will be randomized 1:1:1 to 1 of the following 3 treatment arms:

- Intravitreal ranibizumab $0.2 \mathrm{mg}$ to both eyes on the day of treatment (Baseline), with up to 2 re-treatments allowed for each eye if required

- Intravitreal ranibizumab $0.1 \mathrm{mg}$ to both eyes on the day of treatment (Baseline), with up to 2 re-treatments allowed for each eye if required

- Laser ablation therapy to both eyes (at Baseline)

Details of the investigational treatments are provided in Section 5.5.4.

Visits to assess safety and efficacy are scheduled at regular intervals during the follow-up epoch. Assessments to address the primary objective will be performed at Day 169, which is 24 weeks after starting investigational treatment. No interim analysis is planned. 


\section{Figure 3-1 Study Design}

Screening Treatment and Follow-up Epoch
Epoch

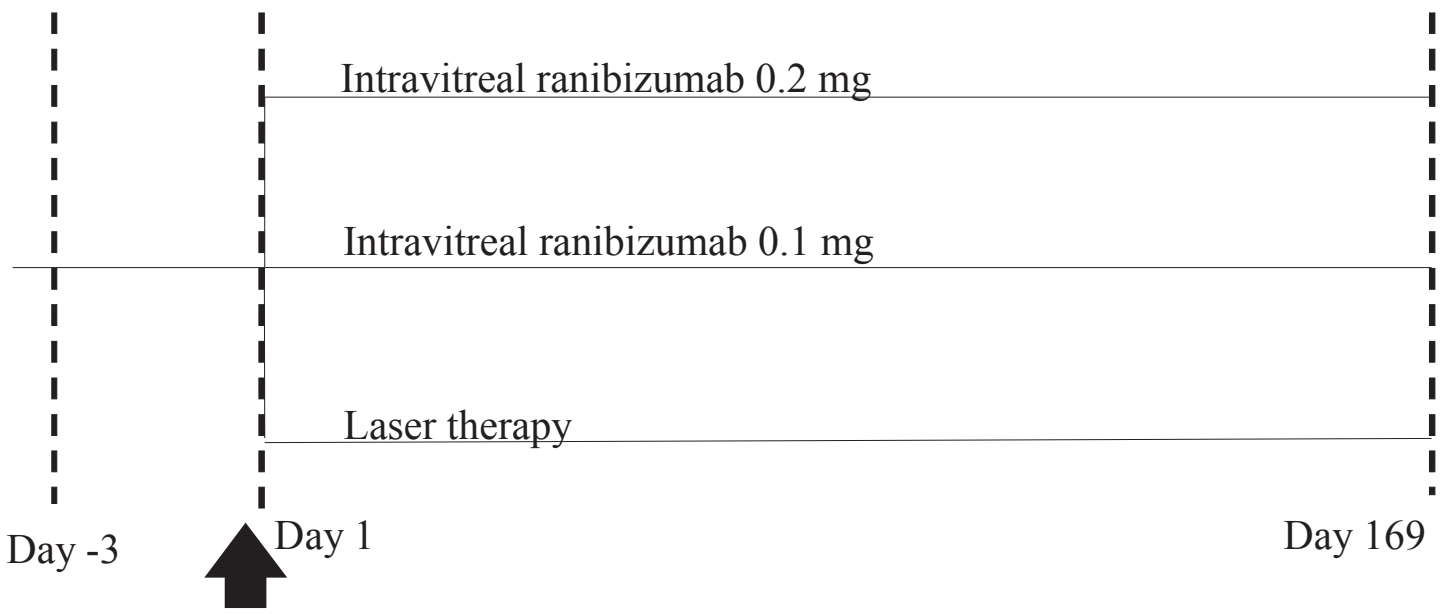

\section{Randomization (1:1:1)}

\subsection{Rationale of study design}

This study will be conducted as open-label. The frequent need for general anesthesia with laser therapy and the presence of laser marks on the treated retina make it impractical to double-mask the study.

Fundus images taken by RetCam digital photography during the study will be sent to a central reading center for archiving and later analysis. The central reading center will not be provided with treatment allocation information and will therefore be masked to the dose of ranibizumab administered.

The eligible ROP types are those recommended for treatment by both the United Kingdom and US guidelines (Royal College of Paediatrics and Child Health 2008, Fierson et al 2013). Patients with zone II stage 2+ ROP are not included in this study as not all cases require immediate treatment and frequent follow-up can sometimes be more appropriate (Royal College of Paediatrics and Child Health 2008).

\subsection{Rationale of dose/regimen, route of administration, and duration of treatment}

Two intravitreal ranibizumab dosages will be investigated: $0.1 \mathrm{mg}$ and $0.2 \mathrm{mg}$ per eye, corresponding to volumes of $10 \mu \mathrm{L}$ and $20 \mu \mathrm{L}$ per eye of a $10 \mathrm{mg} / \mathrm{mL}$ ranibizumab formulation. These doses represent $20 \%$ and $40 \%$, respectively, of the ranibizumab dose administered per eye in a number of adult ocular conditions. With bilateral administration of the doses to be investigated, the total amount of ranibizumab administered to a ROP patient in this study is lower than that administered unilaterally in adult conditions. In comparison, in the BEAT-ROP study in pediatric patients, the dose of bevacizumab was $0.625 \mathrm{mg}$ in $25 \mu \mathrm{L}$ per eye, representing $50 \%$ of the dose commonly administered in each eye in some adult 
ocular conditions (Mintz-Hittner et al 2011). With bilateral administration, the total amount of bevacizumab administered to a patient in the BEAT-ROP study was equivalent to that administered unilaterally in adults for ocular conditions in which intraocular bevacizumab is administered.

The 2 ranibizumab doses chosen allow the evaluation of likely safe and efficacious doses in patients with ROP, based on modeling and simulation to assess the benefit-risk using predicted exposure levels and a PK model that is scaled for infants. The model assumes that:

- Potential efficacy increases when ocular exposure (as estimated by vitreous AUC) increases

- Potential toxicity increases when systemic exposure (as estimated by serum AUC and maximum serum concentration $(\mathrm{Cmax}))$ increases

Ocular exposure in neonates after intravitreal ranibizumab $0.2 \mathrm{mg}$ is expected to be similar to that seen in the treatment of age-related macular degeneration in adults (i.e., ranibizumab $0.5 \mathrm{mg}$ ) and similar to the ocular exposure estimated by the model following intravitreal bevacizumab $0.625 \mathrm{mg}$, as was administered in the BEAT-ROP study (Mintz-Hittner et al 2011) (Figure 3-2). Therefore, an intravitreal ranibizumab dose of $0.2 \mathrm{mg}$ is associated with an ocular exposure that has shown clinical efficacy in adults and in patients in the BEAT-ROP study. 


\section{Figure 3-2 Ocular Exposure Estimated by Area Under the Curve in Vitreous}

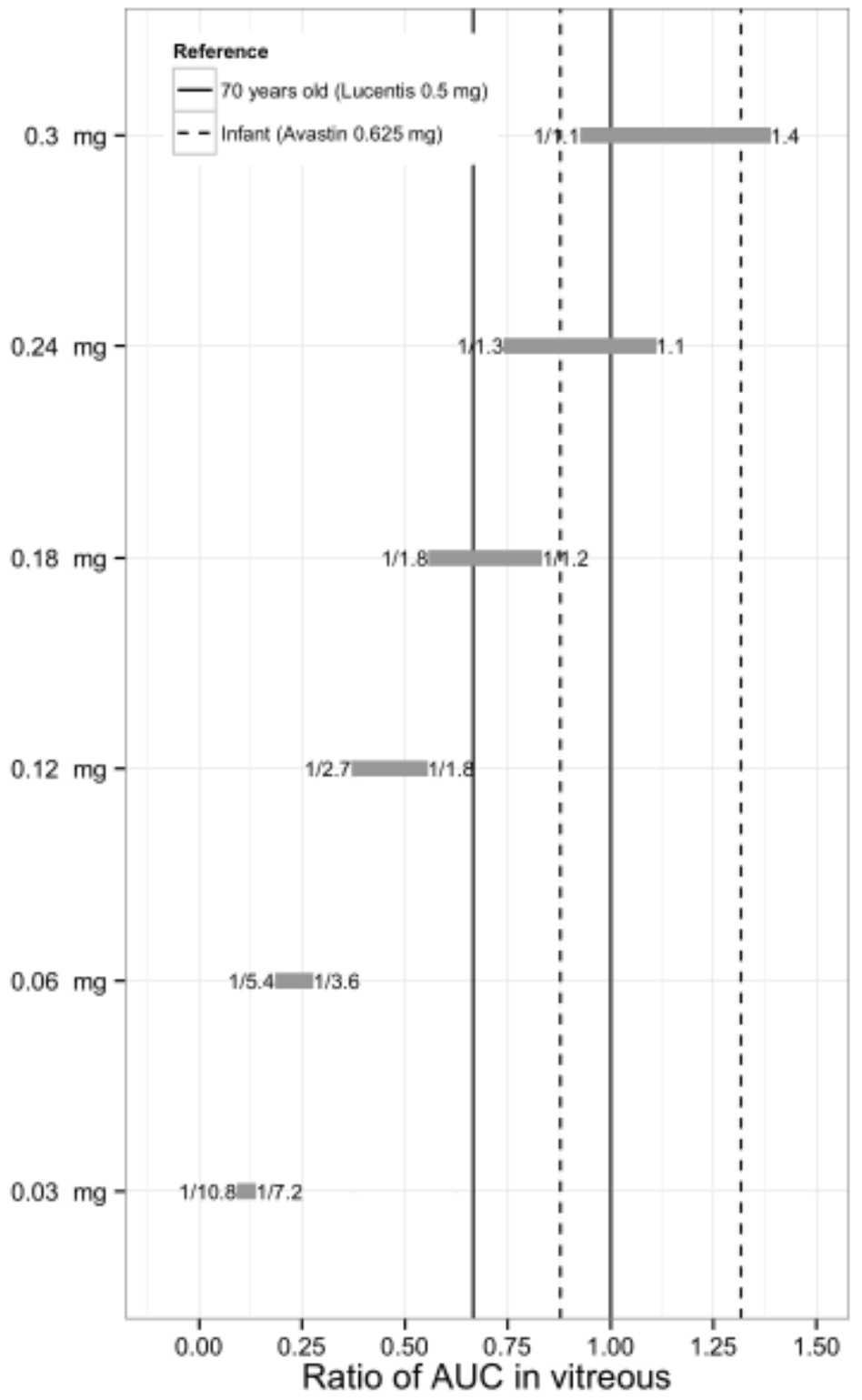

Legend: Horizontal bars - estimated ranibizumab ratio; solid vertical lines - ranibizumab 0.5 mg in adult; dashed vertical lines - bevacizumab $0.625 \mathrm{mg}$ in infant Abbreviation: AUC - area under the curve

Systemic exposure in neonates after bilateral intravitreal ranibizumab with doses as low as $0.1 \mathrm{mg}$ was estimated to be greater than that seen in adult patients. However, bilateral intravitreal ranibizumab doses up to $0.3 \mathrm{mg}$ were estimated to have less systemic exposure than that following bilateral bevacizumab $0.625 \mathrm{mg}$, as was administered in the BEAT-ROP study (Mintz-Hittner et al 2011) (Figure 3-3 and Figure 3-4). Although there is no clear association between systemic exposure and clinical adverse effects, it would be desirable to minimize systemic ranibizumab exposure in the vulnerable premature neonatal population. 


\section{Figure 3-3 \\ Systemic Exposure Estimated by Area Under the Curve in Serum}

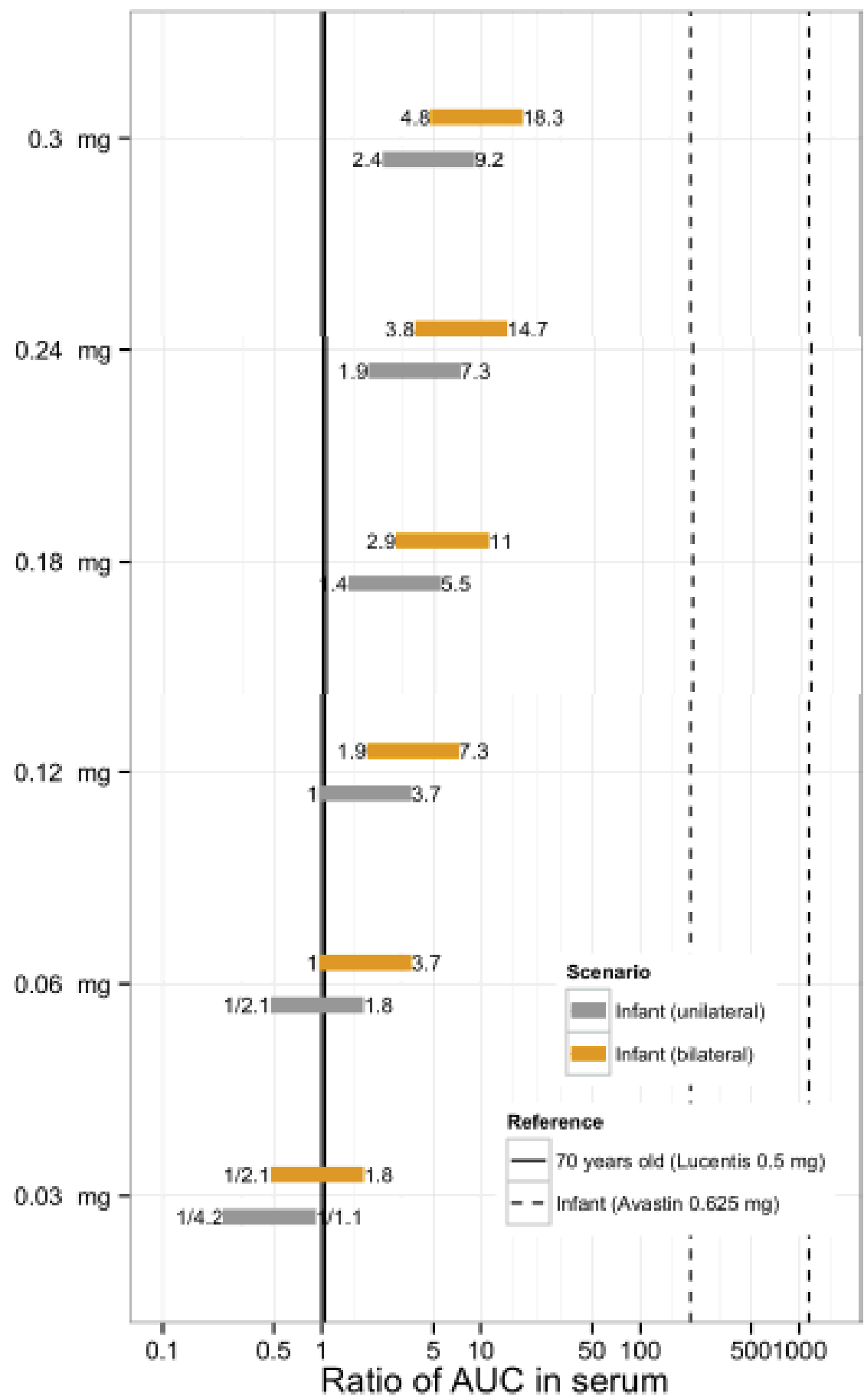

Legend and Abbreviations: Please see under Figure 3-4 


\section{Figure 3-4 Systemic Exposure Estimated by Maximum Concentration in Serum}

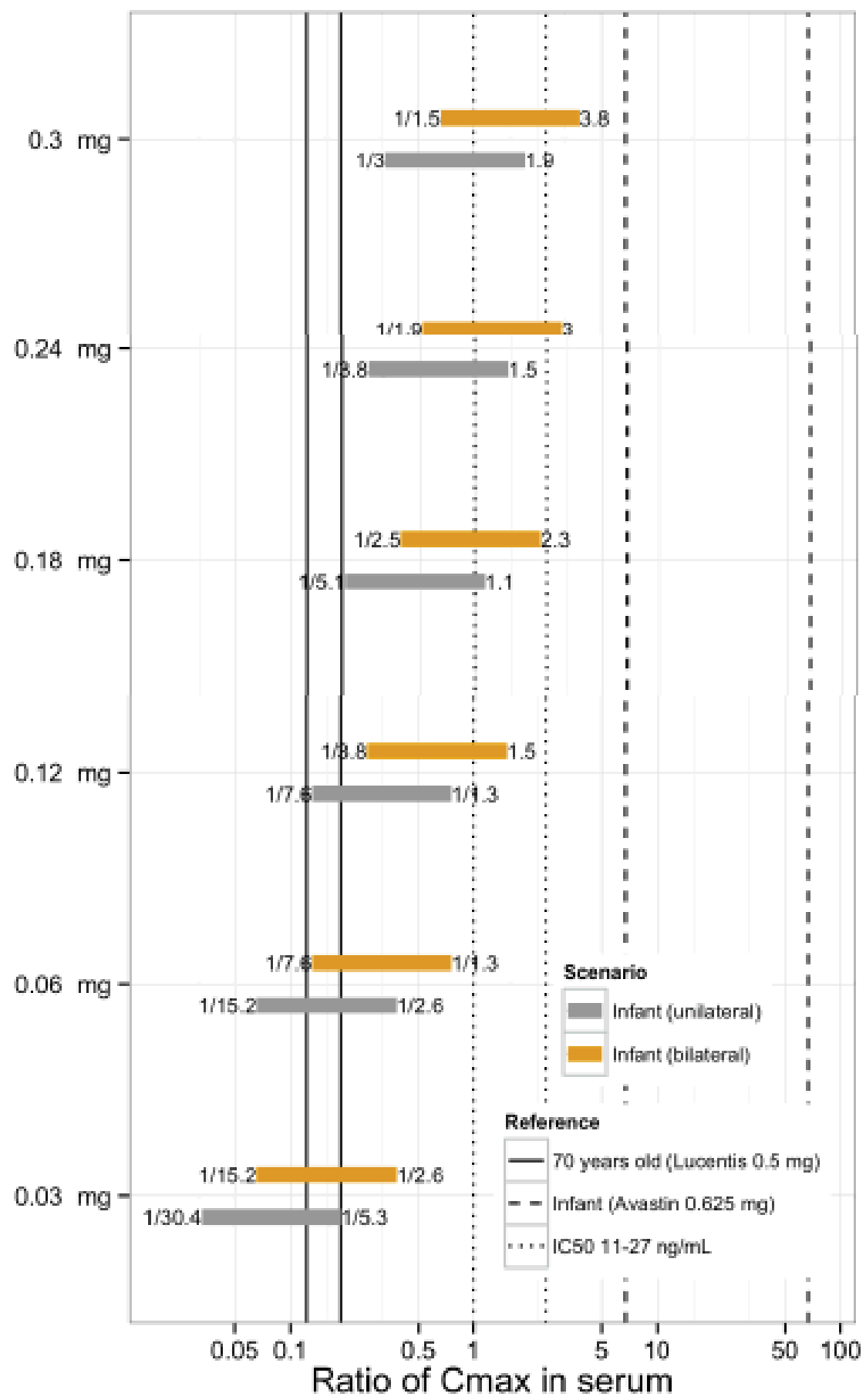

Legend: Horizontal bars - estimated ranibizumab ratio; solid vertical lines - ranibizumab 0.5 mg in adult; dashed vertical lines - bevacizumab $0.625 \mathrm{mg}$ in infant; dotted vertical lines - concentration associated with half-maximal inhibition of vascular endothelial growth factor in vitro

Abbreviations: AUC - area under the curve; Cmax - maximum serum concentration; $\mathrm{IC}_{50}$ - drug concentration causing $50 \%$ inhibition 
Despite acknowledged uncertainties of the modeling and simulation approach, it is estimated that bilateral intravitreal ranibizumab doses of $0.1 \mathrm{mg}$ and $0.2 \mathrm{mg}$ have acceptable benefit-risk ratios based on clinical interpretation of the predicted ocular and systemic exposures. There have been a number of published reports of ranibizumab monotherapy, with doses between $0.2 \mathrm{mg}$ and $0.3 \mathrm{mg}$ per eye, successfully treating ROP (Castellanos et al 2013, Hoerster et al 2013, Lin et al 2012). There have also been some reports of clinical efficacy in ROP with intravitreal bevacizumab doses as low as $20 \%$ of the adult dose $(0.25 \mathrm{mg}$, in combination with laser therapy) and $30 \%$ of the adult dose ( $0.375 \mathrm{mg}$ monotherapy) (Harder et al 2011, Kim et al 2014). Therefore, evaluating anti-VEGF doses lower than that in the majority of published reports may lead to an efficacious regimen with less risk of adverse effects.

Infants who have bilateral ROP that meet the criteria for requiring treatment in both eyes will be enrolled in the study. They will receive either bilateral laser or bilateral ranibizumab treatment at Baseline. Randomization to investigational treatment is by patient rather than eye, since it is not known whether treatment administered in 1 eye will have effects on the contralateral eye. In addition, the safety data will reflect a more real-world practice where physicians are likely to treat both eyes with the same treatment. Due to the need for sedation for laser treatment for babies randomized to the laser arm, many physicians will treat both eyes to avoid risks of exposing the baby to the possibility of 2 treatment sessions in close proximity. Infants with bilateral disease represent more than $80 \%$ of the infants with ROP (Cryotherapy for Retinopathy of Prematurity Cooperative Group 1988) and this study will therefore enable the safety evaluation of the most common ocular involvement pattern and provide data on bilateral intravitreal ranibizumab administration.

Patients randomized to receive ranibizumab will be administered ranibizumab to each eye at Baseline. These patients will receive up to a maximum of 2 ranibizumab re-treatments for either eye to treat worsening ROP at least 28 days after previous ranibizumab treatment in that eye. The ranibizumab dose for re-treatment will be the same as used initially for the individual patient. Only the eye with worsening of ROP will be re-treated. If both eyes demonstrate these signs, then both eyes will be re-treated with ranibizumab.

\subsection{Rationale for choice of comparator}

Laser ablation of the peripheral avascular retina is the current standard of care treatment for ROP (Royal College of Paediatrics and Child Health 2008, Hartnett and Penn 2012). Therefore, this treatment modality has been selected as the comparator for intravitreal ranibizumab in the clinical study.

\subsection{Purpose and timing of interim analyses/design adaptations}

No interim analyses are planned for this study.

\subsection{Risks and benefits}

The risk to patients in this trial will be minimized by compliance with the eligibility criteria, study procedures, and close clinical monitoring. 
This study will be conducted in preterm neonates, which make up a vulnerable population due to their age and associated comorbidities. To protect their rights, safety, and well-being, only study centers and investigators experienced in managing these patients will be invited to participate in the study.

Both investigational treatments are associated with potential risks and benefits. Ablation of the peripheral avascular retina using transpupillary laser therapy is the current standard of care treatment for ROP (Royal College of Paediatrics and Child Health 2008, Hartnett and Penn 2012). However, it may be associated with complications such as bleeding and retinal detachment in the posterior segment and inflammation and cataract formation in the anterior segment (Houston et al 2013). In the long term, laser therapy may be associated with the development of high myopia and it has been reported that $14.3 \%$ of ROP patients treated with ablation therapy developed unfavorable visual acuity outcomes at 9 months (Good 2004, Houston et al 2013). In addition, general anesthesia is often required and this can be associated with a number of risks, especially in the very young and those with concurrent medical conditions. The risk of laser therapy will be minimized in this study by only selecting study centers and investigators experienced in using laser therapy in the treatment of ROP.

The potential benefits of intravitreal anti-VEGF in the management of ROP have been previously described (Section 1.1). Notably, a prospective randomized clinical study demonstrated that intravitreal bevacizumab, compared to laser therapy, was more efficacious in the treatment of ROP and was associated with less myopia in the long term (Mintz-Hittner et al 2011, Geloneck et al 2014). Some potential risks with the use of intravitreal anti-VEGF agents in these patients, as identified in published case reports, include the development of late retinal detachment and reduction in systemic VEGF levels (Honda et al 2008, Jang et al 2010, Lee et al 2010, Zepeda-Romero et al 2010, Hu et al 2012, Sato et al 2012, Hoerster et al 2013). Furthermore, risks associated with the intravitreal injection procedure include endophthalmitis, retinal detachment, traumatic cataract, and increased intraocular pressure.

Serum VEGF levels in ROP infants following ranibizumab have been measured in two studies (Hoerster et al 2013 and Zhou et al 2016). Hoerster et al. describe one infant whose VEGF levels were reduced 1 week after ranibizumab injection, were further reduced below detection limit at week 3 and returned to normal levels at week 4. Zhou et al. measured plasma VEGF levels before and 1 day after ranibizumab injection in 5 ROP infants: plasma VEGF levels were reduced to between $9 \%$ and $37 \%$ of pre-dose VEGF levels 1 day after the injection and normalized after 1 week.

Potential risks of systemic inhibition of VEGF include disturbances of organ and neurological development, renal impairment, bone abnormalities, and thromboembolic events. These effects were observed in studies using animal knockout models, in nonclinical and clinical studies with high systemic doses of anti-VEGF agents or in clinical studies of intravitreal antiVEGF in adults with predisposing comorbidities. No such systemic adverse effects have been reported in the published reports of intravitreal anti-VEGF therapy in ROP. Nonetheless, all patients will be monitored during the study for any new onset AEs.

As with all therapeutic proteins, there is a potential for immunogenicity with ranibizumab. In adult patients, the pretreatment incidence of immunoreactivity to ranibizumab was $0 \%$ to $5 \%$. After monthly dosing with ranibizumab for 6 to 24 months, antibodies to ranibizumab were 
detected in approximately $1 \%$ to $9 \%$ of patients. The clinical significance of immunoreactivity to ranibizumab is unclear. Among neovascular age-related macular degeneration patients with the highest levels of immunoreactivity, some were noted to have iritis or vitritis. Intraocular inflammation was not observed in the retinal vein occlusion or diabetic macular edema patients with the highest levels of immunoreactivity. The number of administrations of ranibizumab in this study is less than that for the treatment of adult conditions. In addition, neonates have a less developed adaptive immune response (Adkins et al 2004). Hence, the potential risk of immunogenicity in this study population is likely to be low.

Patients participating in this study will undergo blood collection. The discomfort and risks associated with blood collection will be minimized by allowing the samples to be collected at the same time as routine clinical samples, when possible. The volume of blood collected will be the minimum required for assay conduct.

An independent Data Monitoring Committee (DMC) will be established to monitor the safety of the trial participants, to ensure that the trial is being conducted with the highest scientific and ethical standards, and make appropriate recommendations based on the data seen.

\section{Population}

The study population will consist of male and female preterm infants with bilateral ROP who require treatment. Approximately 180 patients are planned to be randomized in the study to provide at least 48 evaluable patients in each of the 3 treatment arms, i.e., at least 144 evaluable patients in total.

A patient who drops out after they have been randomized but prior to receiving any investigational treatment will be replaced by enrolling a new patient and assigning a new patient number and a new randomization number.

\subsection{Inclusion criteria}

Patients eligible for inclusion in this study have to fulfill all of the following criteria prior to receiving the first investigational treatment:

1. Signed informed consent from parent(s) or legal guardian(s), in compliance with local requirements

2. Male or female preterm infants with a birth weight of less than $1500 \mathrm{~g}$

3. Bilateral ROP with 1 of the following retinal findings in each eye:

- Zone I, stage $1+, 2+, 3$ or $3+$ disease, or

- Zone II, stage 3+ disease, or

- AP-ROP

\subsection{Exclusion criteria}

Patients fulfilling any of the following criteria prior to receiving the first investigational treatment are not eligible for inclusion in this study. No additional exclusions may be applied by the Investigator, in order to ensure that the study population will be representative of all eligible patients. 
Investigational treatment not clinically appropriate for the following patients:

1. Have ROP disease characteristic in either eye other than that listed in Section 4.1 at the time of the first investigational treatment

2. Have a history of hypersensitivity (either the patient or the mother) to any of the investigational treatments or to drugs of similar chemical classes

Risk of confounding efficacy and/or safety assessments in the following patients:

3. Have received any previous surgical or nonsurgical treatment for ROP (e.g., ablative laser therapy or cryotherapy, vitrectomy)

4. Have been previously exposed to any intravitreal or systemic anti-VEGF agent (either the patient or the mother during this child's pregnancy)

5. Have used (either the patient or the mother) other investigational drugs as part of another clinical study (other than vitamins and minerals) within 30 days or within 5 half-lives of the other investigational drug, whichever is longer

6. Have ocular structural abnormalities that are assessed by the Investigator to have a clinically significant impact on study assessments

7. Have active ocular infection within 5 days before or on the day of first investigational treatment

8. Have a history of hydrocephalus requiring treatment

9. Have a history of any other neurological conditions that are assessed by the Investigator to have a significant risk of severe impact on visual function

10. Have any other medical conditions or clinically significant comorbidities or personal circumstances that are assessed by the Investigator to have a clinically relevant impact on study participation, any of the study procedures, or on efficacy assessments (e.g., poor life expectancy, pupil not able to be adequately dilated, unable to comply with the visit schedule)

\section{$5 \quad$ Treatment}

\subsection{Protocol requested treatment}

\subsubsection{Investigational treatment}

- $0.1 \mathrm{mg}$ or $0.2 \mathrm{mg}$ ranibizumab

Ranibizumab solution for injection will be supplied (open label) in vials. Each vial contains ranibizumab in the concentration of $10 \mathrm{mg} / \mathrm{mL}$ (RFB002 $0.5 \mathrm{mg} / 0.05 \mathrm{~mL}$ ). Ranibizumab is formulated as a sterile solution aseptically filled in a sterile glass vial for single use only. The content of the vial must not be split. The vials will be supplied to each study center by Novartis.

Ranibizumab must be stored according to the label instructions and it must be kept in a secure locked facility.

Each vial will be labeled with the appropriate information. Medication labels will comply with the legal requirements and will be printed in the local language. The storage conditions for study drug will be described on the medication label. 
Ranibizumab that is required for re-treatment (in the ranibizumab arms) or for switched therapy (in the laser arm) will also be provided by Novartis Drug Supply Management.

Novartis will provide sufficient supplies of ranibizumab for treatment use to allow for completion of the study.

- conventional laser ablation therapy

Treatment administration will be detailed in Section 5.5.4 of this protocol.

\subsubsection{Additional treatment}

No additional types of treatment, beyond the investigational treatments (i.e., ranibizumab and laser therapy), are allowed in this trial.

\subsection{Treatment arms}

Patients will be randomized to 1 of the following 3 treatment arms in a ratio of 1:1:1, stratified by ROP zone (in the worst eye according to Table 9-1) and by geographic region:

- Intravitreal ranibizumab $0.2 \mathrm{mg}$ to both eyes on the day of first treatment, with up to 2 re-treatments allowed for each eye if required

- Intravitreal ranibizumab $0.1 \mathrm{mg}$ to both eyes on the day of first treatment, with up to 2 re-treatments allowed for each eye if required

- Laser ablation therapy to both eyes on the day of first treatment, with multiple supplementary laser treatments if necessary

Details of the investigational treatments are provided in Section 5.5.4.

\subsection{Treatment assignment, randomization}

At Visit 1, all eligible patients will be randomized via Interactive Response Technology (IRT) to 1 of the 3 treatment arms. After confirming that the patient fulfills all the inclusion/exclusion criteria, the Investigator or his/her delegate will contact the IRT. The IRT will assign a randomization number to the patient, which will be used to link the patient to a treatment arm (i.e., laser treatment arm or ranibizumab $0.1 \mathrm{mg}$ arm or ranibizumab $0.2 \mathrm{mg}$ arm) and will specify (at Visit 2) either unique medication numbers for the first packages of investigational treatment to be dispensed to the patient in the ranibizumab treatment arms or notification to proceed with laser treatment therapy. The randomization number will not be communicated to the caller.

The randomization numbers will be generated using the following procedure to ensure that treatment assignment is unbiased. A patient randomization list will be produced by the IRT provider's randomization team using a validated system that automates the random assignment of patient numbers to randomization numbers. These randomization numbers are linked to the different treatment arms, which in turn are linked to medication numbers. A separate medication list will be produced by or under the responsibility of Novartis Drug Supply Management using a validated system that automates the random assignment of medication numbers to study drug packs (medication kits). 
Randomization will be stratified by ROP zone (in the worst eye according to Table 9-1) as this disease characteristic may impact treatment outcome. Additionally, randomization will be stratified by geographic region to provide treatment balance within each area that may have different local clinical practices for the management of premature neonates.

After randomization at Visit 1, the treatment allocation cannot be changed.

The randomization scheme for patients will be reviewed and approved by a member of the Biostatistics Quality Assurance group.

\subsection{Treatment masking}

This is an open-label study. The investigators and patients/parents/legal guardians are unmasked to the treatments administered. However, in order to minimize the potential impact of treatment knowledge, randomization data and aggregated statistical analysis done by treatment will be kept confidential from the CTT, until the database is locked for the primary analysis. Further measures to minimize bias related to treatment knowledge will be provided in the Data Management Plan.

Fundus images taken by RetCam digital photography during the study will be sent to a central reading center for archiving and later analysis. The reading center will not be provided with treatment allocation information and will therefore be masked to the dose of ranibizumab administered.

\subsection{Treating the patient}

\subsubsection{Patient numbering}

Each patient is uniquely identified in the study by a combination of his/her center number and patient number. The center number is assigned by Novartis to the investigative site. Upon the parent(s) or legal guardian(s) signing the informed consent form, the patient is assigned a patient number by the Investigator. At each site, the first patient is assigned patient number 1 , and subsequent patients are assigned consecutive numbers (e.g., the second patient is assigned patient number 2, the third patient is assigned patient number 3). The Investigator or his/her staff will contact the IRT and provide the requested identifying information for the patient to register them into the IRT. Only the assigned patient number should be entered in the field labeled "Patient ID" on the electronic data capture (EDC) data entry screen (e.g., enter '1,' '2,' etc). Once assigned to a patient, the patient number will not be reused. If the patient fails to be randomized for any reason, the IRT must be notified within 2 days that the patient was not randomized. The reason for not being randomized is to be entered on the Screening Log, and the Demography/eCRF must also be completed.

\subsubsection{Dispensing the investigational treatment}

Each study site will be supplied by Novartis with (open-label) investigational treatment (ranibizumab only). The study drug packaging has a 2-part label. A unique medication number (kit number) is printed on each part of this label which corresponds to 1 of the 2 ranibizumab treatment arms. The investigator will identify the study drug package to 
dispense to the patient by contacting the IRT and obtaining the medication number (kit number) as well as the treatment arm assignment.

Immediately before dispensing the package to the patient, investigator staff will detach the outer part of the label from the packaging and affix it to the source document (Drug Label Form) for that patient's unique patient number.

The investigator/site personnel are responsible for ensuring that no other person will have access to the medication and drug administration documentation.

Immediately before dispensing and administering ranibizumab, study site staff will record the medication number, drug name, strength, Packaging Control Number, and expiration date from the label in the source document (Drug Accountability Log) containing that patient's unique patient number.

Before laser ablation, study site staff will record the serial number of the laser onto the source document (Laser Log) containing that patient's unique patient number.

\subsubsection{Handling of study treatment}

\subsubsection{Handling of investigational treatment (ranibizumab)}

Investigational treatment (ranibizumab) must be received by a designated person at the study site, handled and stored safely and properly, and kept in a secured location to which only the Investigator and designees have access. Upon receipt, all investigational treatment must be registered in the IRT system and stored according to the instructions specified on the labels. Clinical supplies are to be dispensed only in accordance with the protocol. Technical complaints are to be reported to the respective Novartis Country Pharma Organization Quality Assurance.

Medication labels will be in the local language and comply with the legal requirements of each country. They will include storage conditions for the investigational treatment but no information about the patient except for the medication number.

The Investigator must maintain an accurate record of the shipment and dispensing of investigational treatment in a Drug Accountability Log. Monitoring of drug accountability will be performed by monitors during site visits or remotely and at the completion of the trial.

At the conclusion of the study, and as appropriate during the course of the study, the Investigator will return all unused investigational treatment, packaging, drug labels, and a copy of the completed Drug Accountability Log to the monitor or to the address provided in the investigator folder at each site.

\subsubsection{Handling of other study treatment}

Not applicable. 


\subsubsection{Instructions for prescribing and administering investigational treatment}

After confirmation of the patient's eligibility, the patient will be randomized 1:1:1 through the IRT to 1 of the 3 treatment arms, and the Investigator will initiate the investigational treatment accordingly.

On day(s) when study or rescue treatment is to be administered, all assessments must be conducted before administration except when specified otherwise in Section 6.

All investigational treatments (i.e., ranibizumab treatment and laser therapy) during the study must be recorded in the Dose Administration Record eCRF.

\section{Ranibizumab $0.1 \mathrm{mg}$ or $0.2 \mathrm{mg}$}

Patients randomized to receive ranibizumab $0.1 \mathrm{mg}$ or $0.2 \mathrm{mg}$ will receive a single dose of intravitreal ranibizumab to each eye on Day 1 (Table 6-2).

Instructions on preparation of the aseptic field, preparation of the ranibizumab for injection, the intravitreal injection technique to minimize the risk of complications in patients, and information on pre- and post-injection procedures, including assessment of central retinal artery status after injection (to monitor for increased intraocular pressure), are provided in the Study Operations Manual.

For patients who receive initial ranibizumab treatment, re-treatment with ranibizumab for either eye will occur for worsening of ROP (as defined in Section 5.5.6) at least 28 days after the previous ranibizumab treatment in that eye. Up to 2 re-treatments with ranibizumab per eye to treat ROP recurrence is allowed. The dose used will be the same as the dose to which the patient was randomized. Only the eye with worsening of ROP will be re-treated. If both eyes have these signs, then both eyes will be re-treated. After re-treatment with ranibizumab, the Additional Assessment Schedule is detailed in Table 6-4. All ranibizumab treatments must be recorded in the IRT system.

Ranibizumab re-treatment is not to be administered to an eye that has developed stage 4 or 5 ROP. The development of any complications of ROP, which is assessed by the Investigator as not suitable for treatment with ranibizumab or laser, should be managed as appropriate and the treatment given documented in the eCRF.

\section{Laser therapy}

Patients randomized to laser therapy will receive laser treatment to each eye on Day 1 (Table 6-3).

Conventional laser ablative therapy will be administered, following anesthesia or sedation. Treatment will be applied with near-confluent laser burn spacing (i.e., 0.5 to 1 burn-width apart) to the entire retina peripheral to the ROP lesion. In some cases, limited targeted laser ablation posterior to the ROP lesion, but immediately adjacent to it, may be appropriate. Treatment should be kept well away from the fovea.

Laser ablation will be as complete as possible. Multiple supplementary laser treatments are allowed for both eyes until 3 days after the Day 8 assessment and such treatments are 
considered part of the complete laser treatment. Any unscheduled assessments or visits related to the supplementary laser treatment will be captured in the eCRF.

At the Day 2 and Day 4 assessments, the investigator will decide if supplementary laser treatment is necessary for any eye (e.g., to fill skip lesions, re-treat under-treated areas). Supplementary laser treatment must be performed within the next 3 days of the investigator's decision to treat again. Similarly, at the Day 8 assessment, if the investigator decides that supplementary laser treatment is necessary to any eye, this treatment must be administered within 3 days. However, subsequent to this supplementary treatment, no further laser treatment is allowed for the patient. If at the Day 8 assessment the investigator decides that supplementary laser treatment is not necessary for either eye, no further laser treatment is allowed for the patient from this time point onwards.

\subsubsection{Permitted dose adjustments and interruptions of investigational treatment}

The timing and dose of the investigational treatments are described in Section 5.5.4 and Section 5.5.6. There are no other permitted dose adjustments or interruptions of investigational treatment.

\subsubsection{Rescue medication}

For the purpose of determining if a patient fulfills the criteria for ranibizumab re-treatment or switching of investigational treatment, the following definitions will be used:

- Unchanged - No change in ROP disease activity (considering the stage and extent of ROP disease, and the severity and extent of plus disease characteristics)

- Minimally improved - A reduction in ROP disease activity (considering the stage and extent of ROP disease, and the severity and extent of plus disease characteristics), but which is minimal in the opinion of the investigator

- Worsened - An increase in ROP disease activity (considering the stage and extent of ROP disease, and the severity and extent of plus disease characteristics)

\subsubsection{Patients who receive ranibizumab as initial treatment}

For patients who receive initial ranibizumab treatment, switch over to laser treatment will occur in the below situations of unsatisfactory response. Only the eye with ROP that fulfills the below criteria will be treated with laser. If both eyes fulfill the criteria, then both eyes will be treated with laser.

- ROP that remains unchanged or has worsened at the Day 4 assessment compared to before treatment

- ROP that has only minimally improved, is unchanged, or has worsened at the Day 8 assessment compared to before treatment

- ROP that has worsened, compared to the previous assessment, any time after the Day 8 assessment and up to 27 days after the previous ranibizumab treatment in that eye

Laser therapy will occur within 3 days of the ROP fulfilling the above criteria. Use of this switched laser therapy must be recorded in the Dose Administration Record eCRF as well as 
in the IRT system. After this treatment, the schedule of assessments is detailed in Table 6-6. Supplementary laser treatment is allowed as described in Section 5.5.4, taking the day of the switch laser treatment as the reference day. No further investigational ranibizumab treatment can be administered to the eye after it has been switched to laser therapy.

In case of ROP that worsens, compared to the previous assessment, at least 28 days after the previous ranibizumab treatment in that eye, ranibizumab re-treatment will be administered (up to 2 re-treatments per eye) in that eye and is not considered to be rescue treatment (Section 5.5.4).

For patients who receive ranibizumab re-treatment, switch over to laser treatment will occur in the situations of unsatisfactory response as outlined above in Section 5.5.6.1.

If additional treatment is required for an eye that has already received 2 re-treatments of ranibizumab, the patient may be treated with Standard of Care therapy at the investigator's discretion.

If additional treatment is required for an eye that has already been switched over to laser treatment, the patient may be treated with Standard of Care therapy at the investigator's discretion.

Ranibizumab treatment is not to be administered to an eye that has developed stage 4 or 5 ROP (see Section 5.5.4).

\subsubsection{Patients who receive laser therapy as initial treatment}

For patients who receive initial laser therapy, switch over to ranibizumab 0.2-mg treatment will occur in the below situations of unsatisfactory response. Only the eye with ROP that fulfills the below criteria will be treated. If both eyes fulfill the criteria, then both eyes will be treated.

- ROP that has worsened at the Day 8 assessment compared to before treatment and provided laser treatment is complete as judged by the investigator

- ROP that is only minimally improved, unchanged, or worsens at the Day 15 assessment compared to before treatment

- ROP that has worsened, compared to the previous assessment, any time after the Day 15 assessment

Ranibizumab $0.2 \mathrm{mg}$ treatment will occur within 3 days of the ROP fulfilling the above criteria. Treatment with ranibizumab $0.2 \mathrm{mg}$ in this case must be recorded in the Dose Administration Record eCRF as well as in the IRT system. After this treatment, the schedule of assessments is detailed in Table 6-5.

After receiving this first switched ranibizumab $0.2 \mathrm{mg}$ treatment, re-treatment with ranibizumab $0.2 \mathrm{mg}$ for the eye will occur for worsening of ROP (as defined in Section 5.5.6), compared to the previous assessment, at least 28 days after the previous ranibizumab treatment in that eye. Up to 2 such re-treatments with ranibizumab per eye to treat worsening of ROP is allowed. Only the eye with ROP that worsens will be re-treated. If both eyes have these signs, then both eyes will be re-treated. 
Ranibizumab treatment is not to be administered to an eye that has developed stage 4 or 5 ROP (see Section 5.5.4).

If additional treatment is required for an eye that has already received 2 re-treatments of ranibizumab, the patient may be treated with Standard of Care therapy at the investigator's discretion.

If additional treatment is required for an eye that has received ranibizumab within the last 27 days, the eye may be treated with Standard of Care therapy at the investigator's discretion.

\subsubsection{Concomitant treatment}

The Investigator should instruct the patient's parent(s) or legal guardian(s) to notify the study site about any new medications the patient takes after the patient was enrolled into the study. All medications, procedures, and significant nondrug therapies (including physical therapy and blood transfusions) administered after the patient was enrolled into the study must be recorded, including those from primary care and any other hospital.

\subsubsection{Prohibited treatment}

Use of the treatments displayed in Table 5-1 is NOT allowed after the first investigational treatment.

\section{Table 5-1 Prohibited Treatment}

\begin{tabular}{|l|l|}
\hline Medication & Action to be taken \\
\hline $\begin{array}{l}\text { Any intravitreal anti-VEGF agent other than the } \\
\text { investigational drug }\end{array}$ & $\begin{array}{l}\text { Discontinue the patient from the investigational } \\
\text { treatment, as this could confound efficacy and } \\
\text { safety assessments }\end{array}$ \\
\hline Any systemic anti-VEGF agent & $\begin{array}{l}\text { Discontinue the patient from the investigational } \\
\text { treatment, as this could confound efficacy and } \\
\text { safety assessments }\end{array}$ \\
\hline Any other nonsurgical treatment of ROP & $\begin{array}{l}\text { Discontinue the patient from the investigational } \\
\text { treatment as this could confound efficacy and safety } \\
\text { assessments }\end{array}$ \\
\hline $\begin{array}{l}\text { Any surgical treatment of ROP other than } \\
\text { investigational laser treatment (e.g. cryotherapy, } \\
\text { vitrectomy) }\end{array}$ & $\begin{array}{l}\text { Discontinue the patient from the investigational } \\
\text { treatment, as this could confound efficacy and } \\
\text { safety assessments }\end{array}$ \\
\hline $\begin{array}{l}\text { Any other investigational medicinal product as part } \\
\text { of another clinical study, except vitamins and } \\
\text { minerals }\end{array}$ & $\begin{array}{l}\text { Discontinue the patient from the investigational } \\
\text { treatment, as there could be potential interactions } \\
\text { with the investigational treatments and can } \\
\text { confound the efficacy and safety assessments }\end{array}$ \\
\hline
\end{tabular}

Abbreviations: ROP - retinopathy of prematurity; VEGF - vascular endothelial growth factor

\subsubsection{Discontinuation of investigational treatment}

The patient's parent(s) or legal guardian(s) may voluntarily discontinue further investigational treatment for any reason at any time.

The Investigator should discontinue further investigational treatment for a given patient if, on balance, he/she believes that continuation would be detrimental to the patient's well-being.

Further investigational treatment must be discontinued under the following circumstances: 
- Use of prohibited treatment, as specified in Table 5-1

- Any other protocol deviation that results in a significant risk to the patient's safety

Patients who discontinue further investigational treatment should NOT be considered withdrawn from the study. They should return for assessments as described in Table 6-1. If they fail to return for these assessments for unknown reasons, every effort (e.g., telephone, email, letter) should be made to contact their parent(s) or legal guardian(s) as specified in Section 5.5.11.

\subsubsection{Premature patient withdrawal from the study}

If parent(s) or legal guardian(s) decide to prematurely withdraw a patient from the study for any reason, the investigator must make every effort to perform the assessments for the Premature withdrawal visit as described in Table 6-2 and Table 6-3. The Investigator must make every effort (e.g., telephone, email, letter) to determine the primary reason for this decision and record this information on the Study Completion eCRF. The investigator must also contact the IRT to register the patient's discontinuation from the study.

The investigator must provide follow-up medical care for all patients who are prematurely withdrawn from the study, or must refer them for appropriate ongoing care.

Patients who prematurely withdraw from the study will not be replaced.

\subsubsection{Withdrawal of consent}

The patient's parent(s) or legal guardian(s) may voluntarily withdraw consent to participate in the study for any reason at any time.

Withdrawal of consent occurs only when a patient's parent(s) or legal guardian(s) does not want the patient to participate in the study any more, does not want any further visits or assessments, does not want any further study related contacts, and does not allow analysis of already obtained biologic material.

Investigational treatment must be discontinued and no further assessments conducted. All biological material that has not been analyzed at the time of withdrawal must not be used. Further attempts to contact the patient's parent(s) or legal guardian(s) are not allowed unless safety findings require communicating or follow-up. The investigator must also contact the IRT to register the patient's discontinuation from the study.

\subsubsection{Premature patient withdrawal}

The patient must be withdrawn from the study under the following circumstances:

- Any protocol deviation, where continuation in the study may result in a significant risk to the patient's safety

\subsubsection{Loss to follow-up}

For patients whose status is unclear because they fail to appear for study visits and their parent(s) or legal guardian(s) have not communicated an intention to withdraw the patient, the Investigator should show "due diligence" by contacting the patient's parent(s) or legal guardian(s), family, or family physician as agreed in the informed consent and by 
documenting in the source documents steps taken to contact the patient, e.g., dates of telephone calls, registered letters, etc. A patient should not be formally considered lost to follow-up until his/her scheduled end of study visit would have occurred.

\subsubsection{Emergency breaking of assigned treatment code}

Not applicable. This is an open-label study.

\subsubsection{Study completion and post-study treatment}

The study will be considered completed for an individual patient when he or she completes the last scheduled study visit, i.e. Visit 112 or the last additional visit as per protocol or Visit 199 in the case of premature withdrawal, whichever is the latest. The study as a whole will be considered completed when the last patient has had his or her last scheduled study visit.

It is planned to randomize approximately 180 patients in the study, in order to provide at least 48 evaluable patients in each treatment arm. Patients who are being screened when the required number of randomized patients is met will be allowed to continue in the study. Recruitment into the study is not expected to be rapid and, once the approximate number of patients is reached, sites can be closed leaving only those with screens ongoing to continue.

Patients who have received investigational treatment and are prematurely withdrawn from the study will not be replaced; a patient who drops out after they have been randomized but prior to receiving any investigational treatment will be replaced by enrolling a new patient and assigning a new patient number and randomization number (Section 5.5.10.1).

The Investigator must provide follow-up medical care for all patients who are prematurely withdrawn from the study, or must refer them for appropriate ongoing care. All eligible patients will be offered enrollment in an extension study to CRFB002H2301 to monitor long term visual outcomes and safety or local standard of care.

\subsubsection{Early study termination}

The study can be terminated at any time for any reason by Novartis. Should this be necessary, the patient should be seen as soon as possible and treated as for a prematurely withdrawn patient. The Investigator may be informed of additional procedures to be followed in order to ensure that adequate consideration is given to the protection of the patient's interests. The Investigator will be responsible for informing the Institutional Review Board/Independent Ethics Committee (IRB/IEC) of the early termination of the trial.

\section{$6 \quad$ Visit schedule and assessments}

Table 6-1 through Table 6-6 list all of the assessments and indicate with an " $x$ " when the visits are performed. When a visit as per the additional assessment schedule coincides with a visit as per the main assessment schedule, assessments have to be performed as per the main assessment schedule.

Patients should be seen for all visits on the designated day, with an allowed "visit window" as indicated on the relevant assessment schedule tables. 
Patients can also be assessed by the investigator in addition to the visit schedule, if the investigator deems that the additional contact is necessary for the patient's well-being. Such unscheduled visits and assessments will be documented in the eCRF as well as in the investigator's source notes and any information on adverse events will be documented in the eCRF.

At a minimum, patient's parent(s) or legal guardian(s) will be contacted for safety evaluations during the 30 days following the last study visit or following the last administration of investigational treatment (whichever is later) (see Section 7.2). Documentation of attempts to contact the patient's parent(s) or legal guardian(s) should be recorded in the source documentation. 


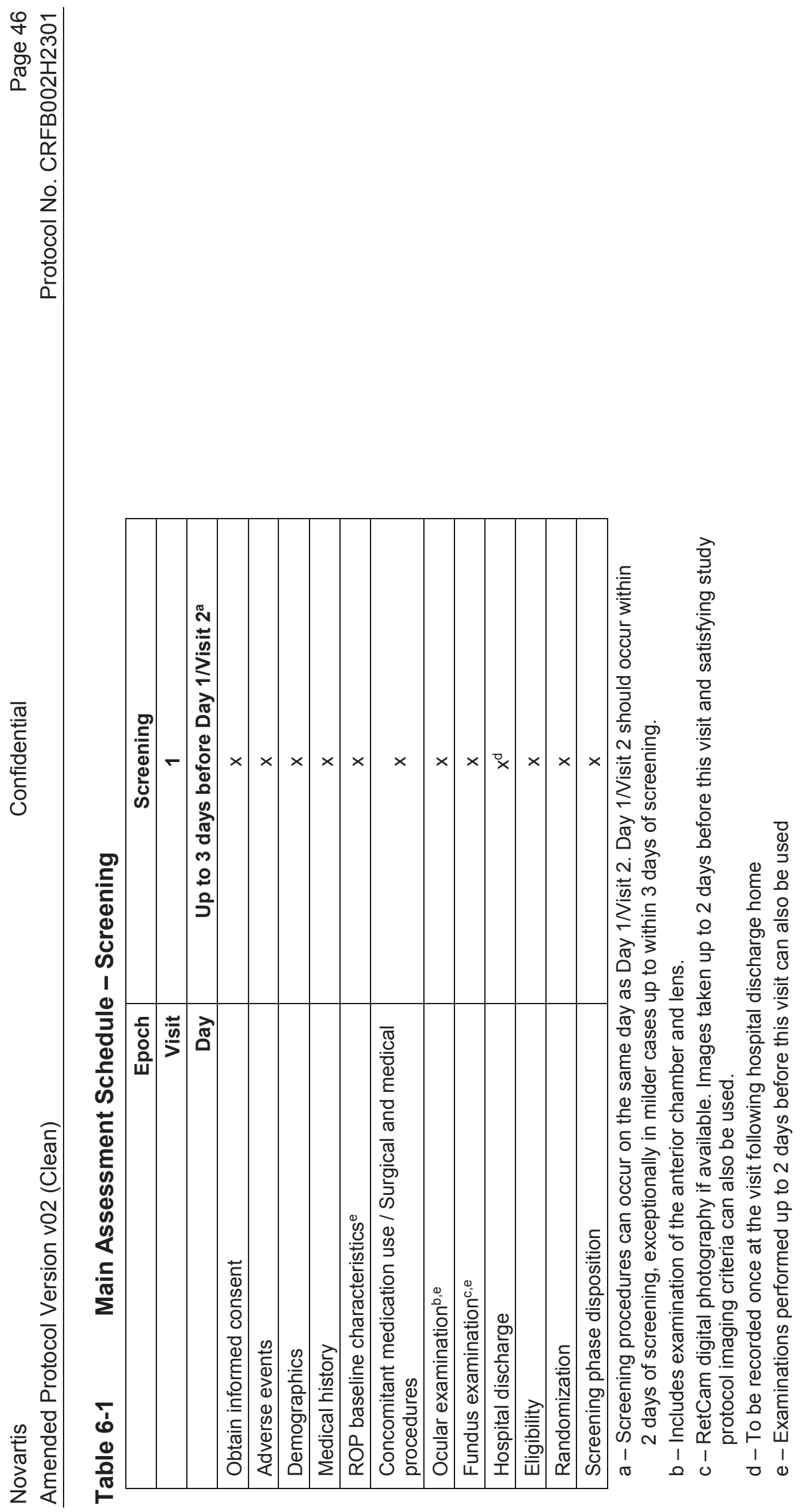




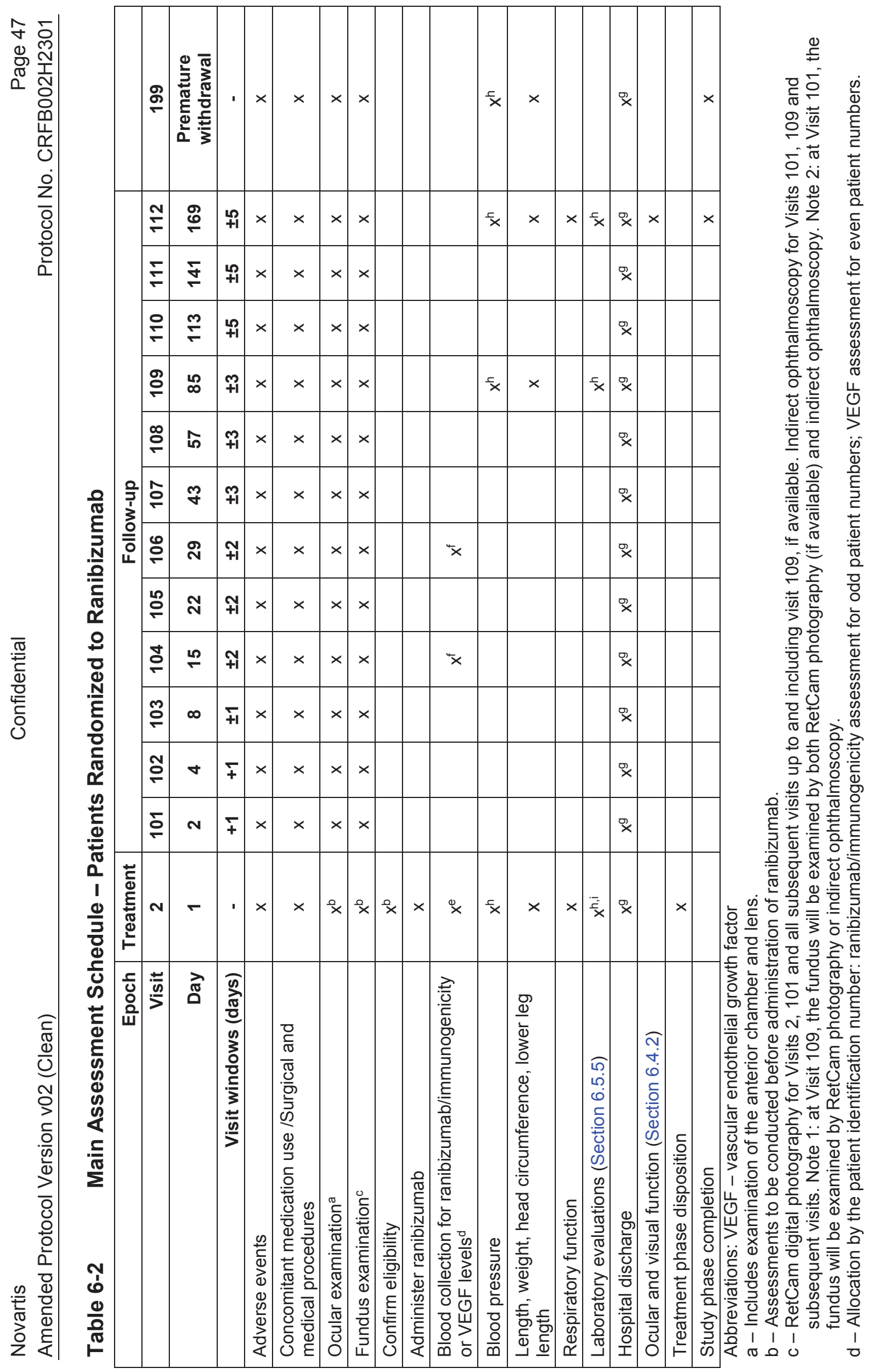




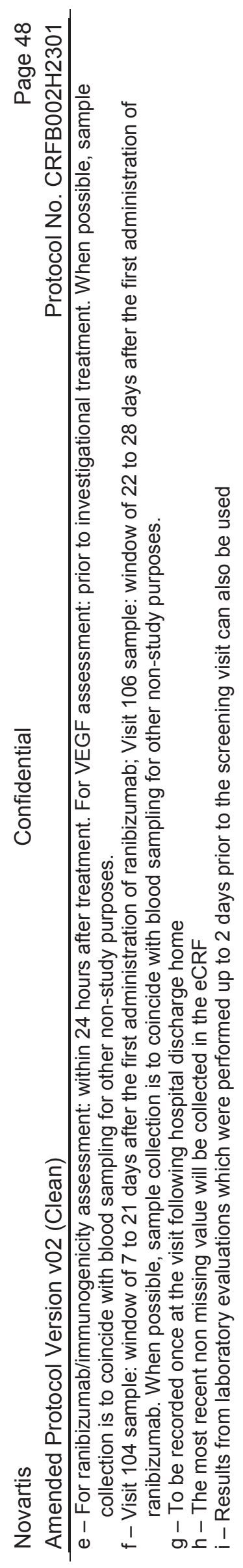




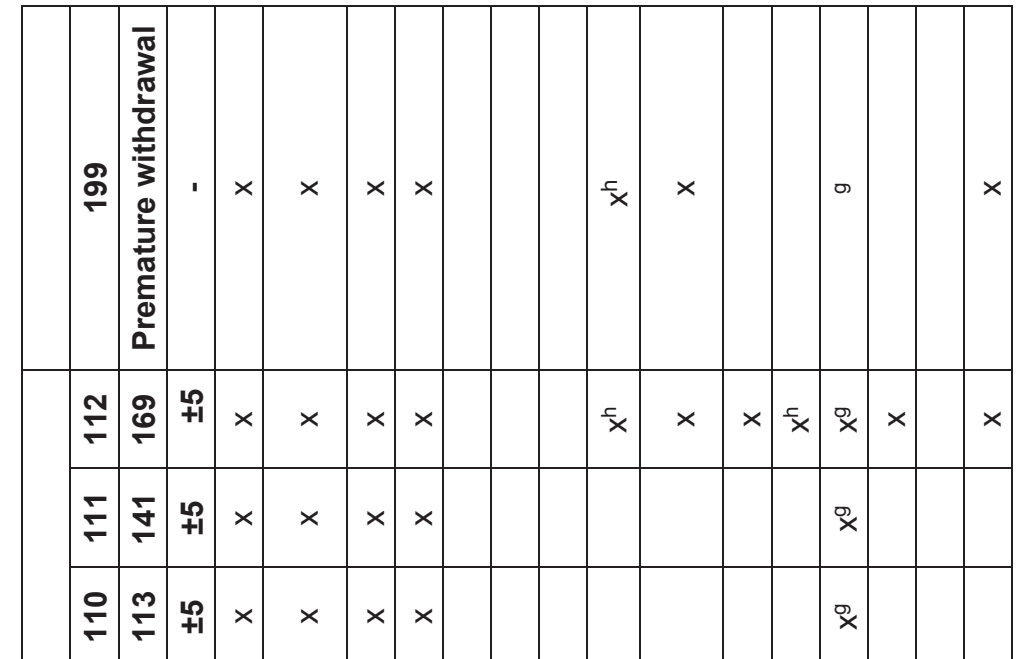

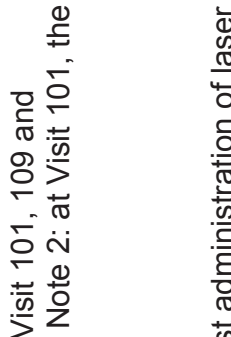

훈

긍

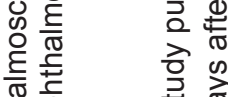

类

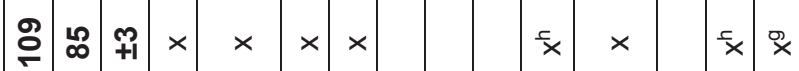

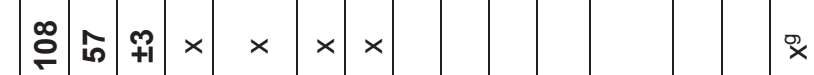

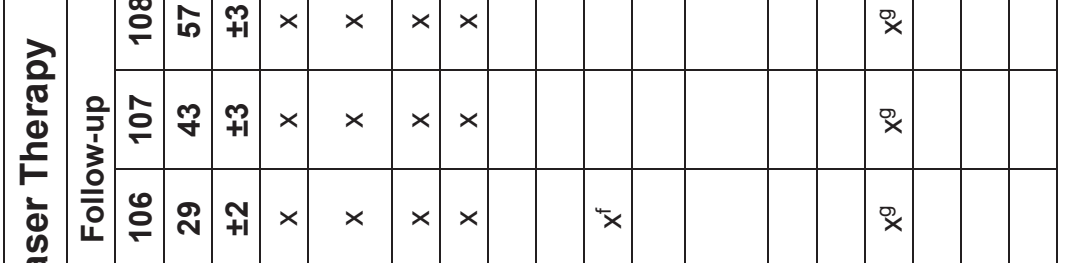

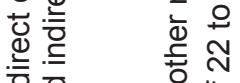

要需

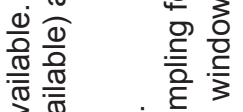

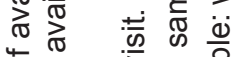

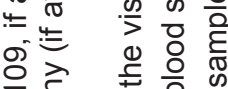

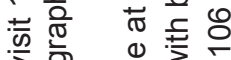

유융 Ð

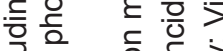

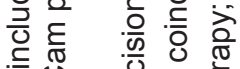

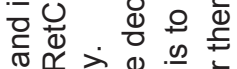

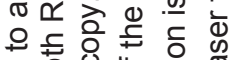

응해

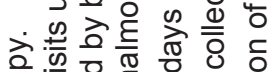

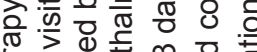

娄

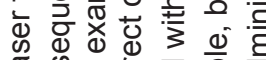

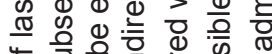
횬 它

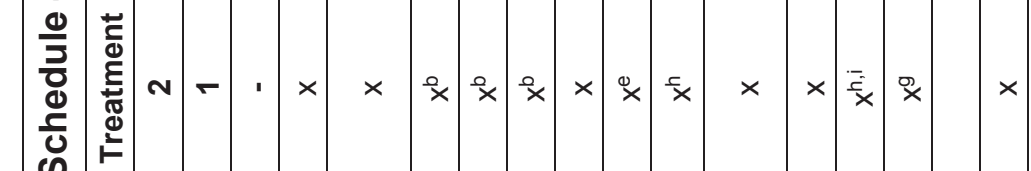

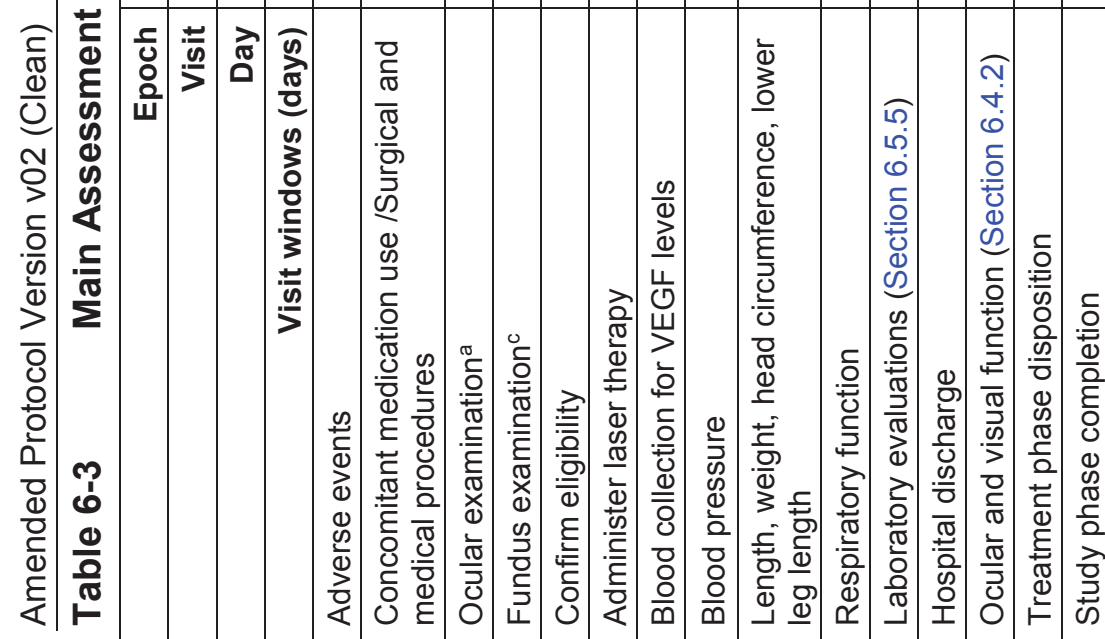

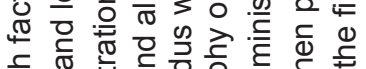

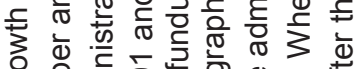
일 需 $\frac{\pi}{0}$ 元

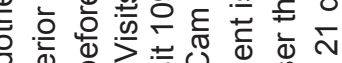

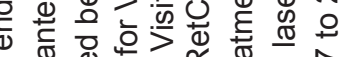

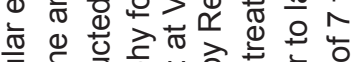

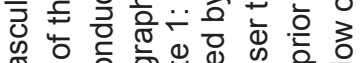

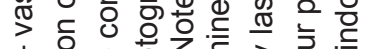

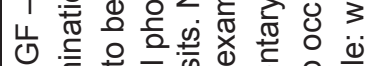

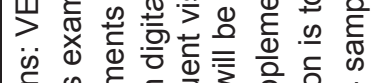
¿0

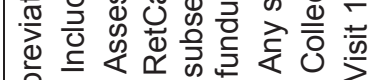

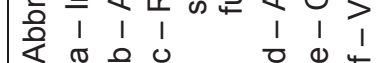




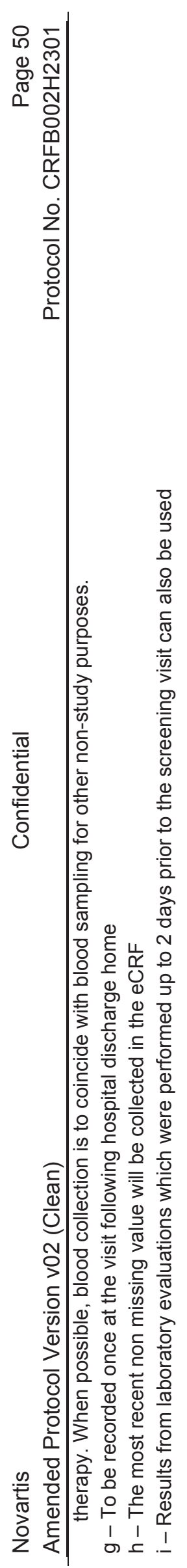




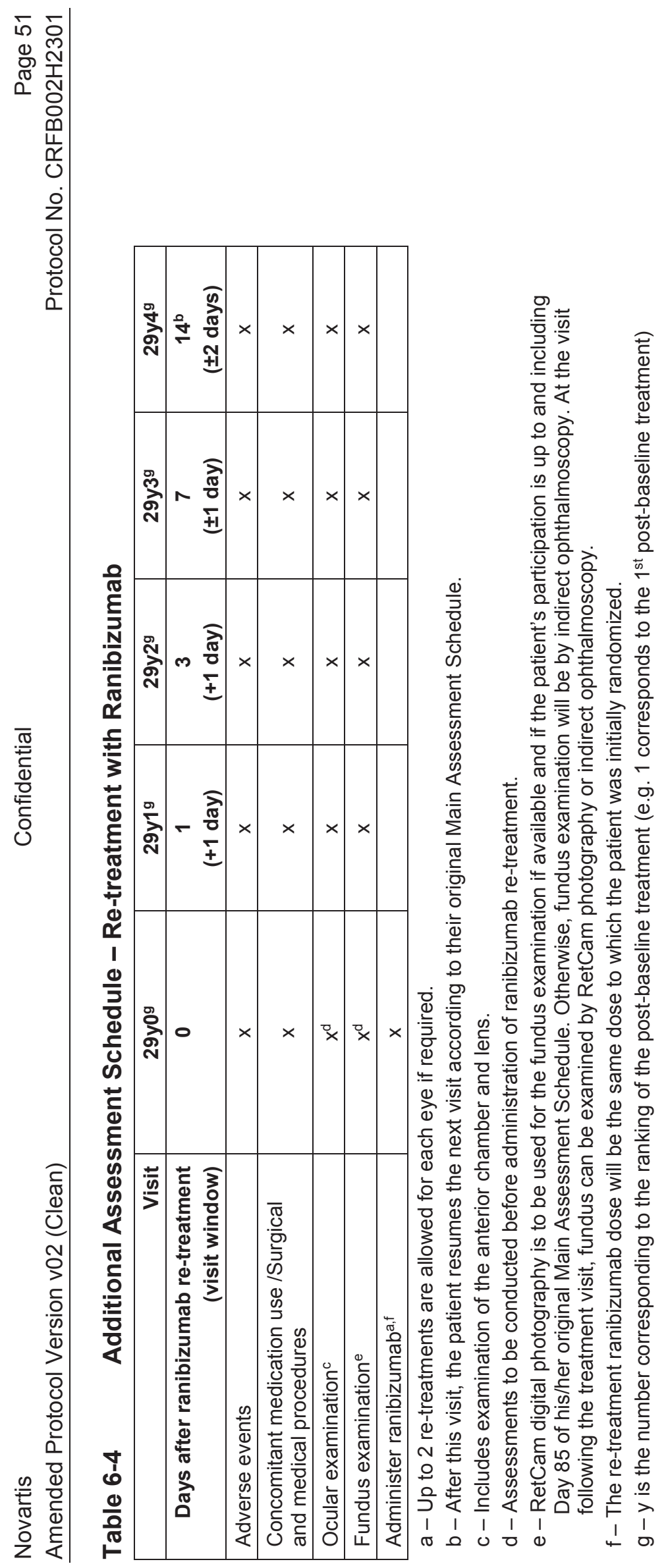




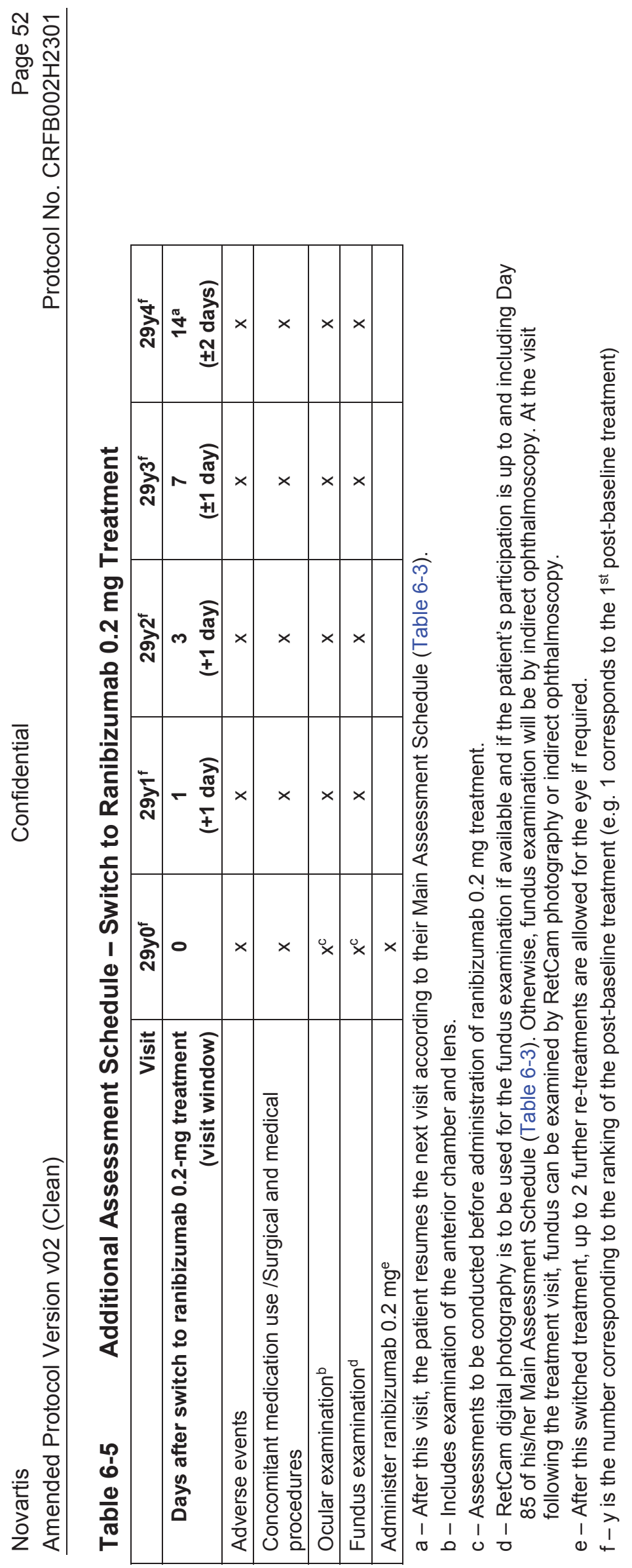




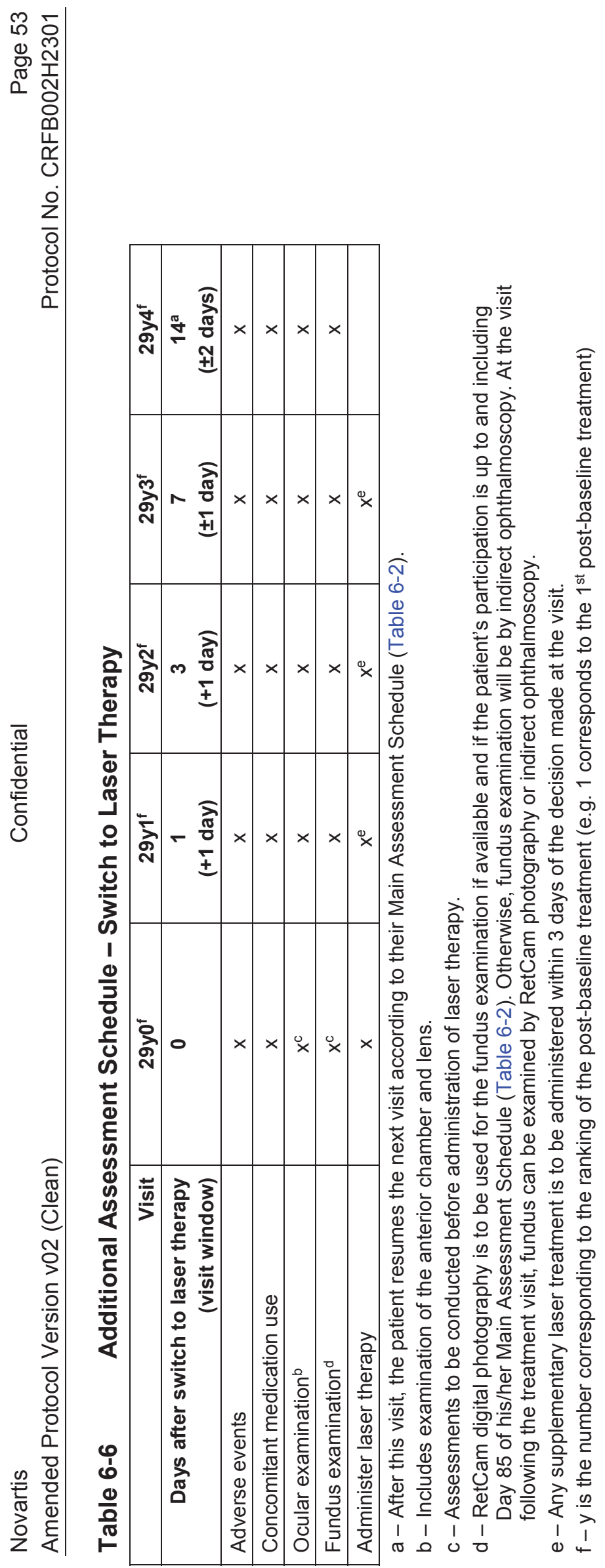




\subsection{Information to be collected on screening failures}

All patients with informed consent obtained from their parent(s) or legal guardian(s) but not treated or randomized will have the study completion page for the screening epoch, demographics, inclusion/exclusion, and serious adverse event (SAE) data collected. Adverse events that are not SAEs will be followed by the Investigator and collected only in the source data.

\subsection{Patient demographics/other baseline characteristics}

Patient demographic and baseline characteristic data to be collected on all patients include: race and ethnicity (based on that of the mother), start date of the mother's last normal menstrual period, sex, year of birth, gestational age at birth (by completed weeks), birth weight, length and head circumference at birth, characteristics of the ROP, and relevant medical history/current medical condition (including multiple pregnancy information, birth hospital, and Apgar (appearance, pulse, grimace, activity, respiration) scores at 1 and 5 minutes, and intubation requirement).

\subsection{Treatment exposure and compliance}

Every time that ranibizumab treatment is to be administered (treatment/re-treatment and rescue), IRT needs to be contacted for the medication (kit) number. For laser therapy, IRT will only be contacted for the first administration (first treatment and rescue) but not for any supplementary laser treatment administered. Information regarding investigational treatment administration will be collected in the Dose Administration Record in the eCRF.

Exposure to ranibizumab treatment will be based on the number of injections by eye and overall (as databased on IRT as well as on the Drug Administration Record eCRF). Compliance with ranibizumab treatment will be assessed by the field monitor at each visit using vial counts and information provided by the pharmacist or by the investigator.

Exposure to laser treatment will be based on the number of days laser treatment is received (baseline and/or supplementary laser treatment) by eye and overall per patient (as databased on IRT as well as on the Drug Administration Record eCRF).

\subsection{Efficacy}

\subsubsection{Ophthalmic fundus features}

The presence of the following fundus features will be assessed in both eyes by the Investigator at all study visits, using images taken by RetCam digital photography and/or as assessed by indirect ophthalmoscopy (for the screening visit only, images taken up to 2 days before the screening visit and satisfying study protocol imaging criteria can also be used; similarly, results of ophthalmic exams performed up to 2 days before the screening visit can also be used for this visit):

- Features of active ROP disease: 
- ROP disease - zone, stage, extent by clock hours, and characteristics of vascular changes of plus disease (extent by quadrants and severity)

- Extra-retinal vessels extending from the retina into the vitreous and judged to be a sign of active ROP disease

- Features of late sequelae of ROP:

- Retrolental membrane obscuring the view of the posterior pole

- Substantial temporal retinal vessel dragging causing abnormal structural features/ macular ectopia

- Posterior retinal fold involving the macula

- Retinal detachment involving the macula

- Retinal detachment not involving the macula

- Pre-retinal fibrosis

- Degree of full retinal vascularization

When Digital photography is performed, the RetCam system will be used. Six standard images will be taken using the $130^{\circ}$ lens:

- Iris shot

- Optic nerve at center of image

- Optic nerve at superior edge of image

- Optic nerve at inferior edge of image

- Optic nerve at nasal edge of image

- Optic nerve at temporal edge of image

Additional information on the photography protocol will be described in the Central Reading Center Manual.

If available, RetCam digital photography is scheduled to be performed at all study visits up to and including the Day 85 visit. If digital photography is not available or is not able to be performed or does not provide adequate visualization of the fundus during these visits, an examination of the fundus will be performed by indirect ophthalmoscopy. Indirect ophthalmoscopy can also be used on the visit following the treatment visit.

Because it can be difficult to perform RetCam digital photography in infants from 3 months of age without sedation, evaluation of fundus features after Day 85 will be by indirect ophthalmoscopy. If available, RetCam digital photography will also be performed, where possible, if the patient is sedated for the administration of any additional ROP treatment after Day 85. Both RetCam digital photography (if available) and indirect ophthalmoscopy are scheduled to be performed at the Day 85 visit to allow the Investigator to assess the fundus using both methods, prior to switching to using indirect ophthalmoscopy only during subsequent study visits.

In addition, images taken by RetCam digital photography during the study will be sent to a central reading center for archiving and later analysis of e.g. the presence of fundus features of active ROP disease and of late sequelae of ROP. 


\subsubsection{Ocular and visual function}

The following will be assessed at Day 169 in both eyes by the Investigator:

- Presence of nystagmus with the eyes in the primary position

- Abnormal fixation behavior (e.g. vision central, steady, maintained)

- Abnormal pupillary light reaction

- Refraction (in spherical equivalence) as assessed by retinoscopy or auto-refraction after cycloplegia

Additional information on the conduct of these assessments will be described in the Study Operations Manual.

\subsubsection{Appropriateness of efficacy assessments}

The fundus features assessed in this study correspond with morphologic evidence of persistence or complications resulting from ROP, and are associated with poor visual outcome. Similar measures have been used in previous studies assessing treatments for ROP (Cryotherapy for Retinopathy of Prematurity Cooperative Group 1988, Good 2004, Mintz-Hittner et al 2011).

\subsection{Safety}

\subsubsection{Ocular examination}

The presence of any other fundus features abnormalities will be assessed in both eyes at all study visits (see Section 6.4.1).

Ocular examination of structures in addition to that of the fundus will be performed at all study visits in both eyes, including assessment of anterior ocular structures and the lens.

For the screening visit, results from ophthalmic exams performed up to 2 days before this visit can be used. Information must be included in the source documentation at the study site.

Clinically significant abnormal findings will be reported as AEs in the eCRF.

\subsubsection{Vital signs}

\subsubsection{Blood pressure}

Blood Pressure measurements are not required by the protocol. Instead, the most recent systolic and diastolic blood pressure $[\mathrm{mm} \mathrm{Hg}]$ measured as part of the routine clinical care should be recorded in the eCRF at visits specified in Section 6.

Information must be included in the source documentation at the study site. Significant findings which meet the definition of an Adverse Event must be recorded on the Adverse Event section of the CRF.

\subsubsection{Length, weight, head circumference and lower leg length}

For the assessment of length, weight, head circumference and lower leg length, the patient should be wearing minimal clothing (e.g., a clean diaper). Hats, shoes, socks, coats, and other 
items of clothing should be removed. Study staff trained in the assessment of infants will perform these assessments.

Recumbent length in centimeters will be measured using an infantometer or according to local clinical practice. Body weight to the nearest gram will be measured using an electronic scale. The maximum occipitofrontal head circumference in centimeters will be measured using a tape measure.

The lower leg length (knee-heel length) will be measured to the nearest 0.1 centimeter using a sliding caliper or according to local clinical practice. Measurements should always be done on the right side, unless a physical deformity affects the right side.

\subsubsection{Respiratory function}

Patients' respiratory function will be assessed by collecting the requirement, if any, for positive pressure support (positive end expiratory pressure (PEEP)) and oxygen supplementation (FiO2). Oxygen saturation will also be collected, if this has been measured as part of routine clinical practice. Data should be recorded on the eCRF.

Information must be included in the source documentation at the study site. Significant findings which meet the definition of an Adverse Event must be recorded on the Adverse Event section of the CRF.

\subsubsection{Monitoring of adverse events (ocular and systemic)}

Patients will be monitored throughout the study for AEs. Please refer to Section 7.1 and Section 7.2 for details on recording AEs and recording and reporting of SAEs, respectively.

In this study, the following safety events will be collected as study endpoints only and are not to be additionally considered or recorded as an AE or SAE. These specific events are captured on the endpoint page of the eCRF only and investigators should not report these on an $\mathrm{AE} / \mathrm{SAE}$ page of the eCRF:

- Substantial temporal retinal vessel dragging causing abnormal structural features/ macular ectopia

- Retrolental membrane obscuring the view of the posterior pole

- Posterior retinal fold involving the macula

- Retinal detachment

- Pre-retinal fibrosis

\subsubsection{Laboratory evaluations}

No additional laboratory evaluations are required per protocol (e.g., hematology, clinical chemistry or urinalysis). Data from tests which were performed as part of routine clinical practice will be collected. At visits specified in Section 6, the most recent laboratory values for the tests below will be collected on the eCRF, where such data are available. Abnormal laboratory test results that meet the criteria of an AE (Section 7.1) must be reported in the AE eCRF. 
For the Day 1 visit, results from laboratory evaluations which were performed up to 2 days prior to the screening visit can also be used. Information must be included in the source documentation at the study site.

\subsubsection{Hematology}

Hematology will include hemoglobin ( $\mathrm{Hb}$ ), white cell count (WCC) and platelet count (PLT).

\subsubsection{Clinical chemistry}

Serum chemistry data will include urea and electrolytes (sodium, potassium, creatinine and urea/blood urea nitrogen $(\mathrm{BUN})$ ) and liver function tests (bilirubin, albumin, total protein, aspartate transaminase (AST), alanine aminotransferase (ALT), alkaline phosphatase (ALP)).

\subsubsection{Urinalysis}

Urinalysis will include urine protein.

\subsubsection{Appropriateness of safety measurements}

The safety assessments selected are standard for this patient population.

\subsection{Other assessments}

To minimize the volume of blood collected, each patient will contribute to either systemic ranibizumab concentration/ anti-ranibizumab antibody level assessment OR systemic VEGF level assessment.

Systemic ranibizumab concentrations/ anti-ranibizumab antibody levels will be assessed in patients who receive initial ranibizumab treatment and with an odd patient identification number.

Systemic VEGF levels will be assessed in patients who receive initial ranibizumab treatment and with an even patient identification number, as well as in all patients who receive initial laser therapy.

Blood sampling for the assessments listed in Section 6.6 must be undertaken wherever possible. In cases where blood collection is not possible, the reason for this should be recorded in the blood collection eCRF.

\subsubsection{Systemic ranibizumab concentration}

For patients who receive initial ranibizumab treatment and with an odd patient identification number, blood samples (Appendix 1) for the determination of ranibizumab concentrations will be collected at the following time points:

- Within 24 hours after the first administration of ranibizumab

- At Day 15, with an allowed time window of 7 to 21 days after the first administration of ranibizumab

- At Day 29, with an allowed time window of 22 to 28 days after the first administration of ranibizumab 
Where possible, timing of the blood collection should coincide with blood collection for other non-study purposes.

A target of $0.8 \mathrm{~mL}$ of blood (with a minimum of $0.5 \mathrm{~mL}$ ) will be collected at each time point into a plain, additive-free serum tube.

The blood sample will be collected and processed according to the Study Operation Manual and the Laboratory Manual respectively. All sample tubes will be labeled with the study number, center and patient identification (patient identification number) and sample number.

\subsubsection{Systemic anti-ranibizumab antibody level}

The serum samples for systemic ranibizumab concentration determination will also be used for anti-ranibizumab antibody level determination, provided the serum sample volume collected is sufficient.

\subsubsection{Systemic vascular endothelial growth factor level}

For patients who receive initial ranibizumab treatment and with an even patient identification number and for all patients who receive initial laser therapy, blood samples (Appendix 2) for the determination of systemic VEGF levels (i.e., pharmacodynamics) will be collected at the following time points:

- Before the first investigational treatment

- At Day 15, with an allowed time window of 7 to 21 days after the first investigational treatment

- At Day 29, with an allowed time window of 22 to 28 days after the first investigational treatment

Where possible, timing of the blood collection should coincide with blood collection for other non-study purposes.

A target of $0.8 \mathrm{~mL}$ of blood (with a minimum of $0.5 \mathrm{~mL}$ ) will be collected at each time point and processed according to the Study Operation Manual and the Laboratory Manual respectively. All sample tubes will be labeled with the study number, center and patient identification (patient identification number), and sample number.

\subsubsection{Hospitalization}

Duration of hospitalization (from birth to hospital discharge home) and weight at discharge from hospital home will be collected.

\section{$7 \quad$ Safety monitoring}

\subsection{Adverse events}

An AE is any untoward medical occurrence (i.e., any unfavorable and unintended sign (including abnormal laboratory findings), symptom, or disease) in a subject or clinical investigation patient after providing written informed consent for participation in the study. 
Therefore, an AE may or may not be temporally or causally associated with the use of a medicinal (investigational) product.

In this study, the following safety events will be collected as study endpoints only and are not to be additionally considered or recorded as an AE or SAE. These specific events are captured on the endpoint page of the eCRF only, and investigators must not report these on an AE/SAE page of the eCRF:

- Substantial temporal retinal vessel dragging causing abnormal structural features/ macular ectopia

- Retrolental membrane obscuring the view of the posterior pole

- Posterior retinal fold involving the macula

- Retinal detachment

- Pre-retinal fibrosis

The occurrence of AEs should be sought by non-directive questioning of the patient's parent(s) or legal guardian(s) at each visit during the study. Adverse events also may be detected when they are volunteered by the patient's parent(s) or legal guardian(s) during or between visits or through physical examination, laboratory test, or other assessments. Study staff, including the Investigator, should make particular effort to follow-up with neonatal intensive care unit staff and the neonatologist (if not also the Investigator) to ensure that no unreported AEs/SAEs have been overlooked.

Abnormal laboratory values or test results constitute AEs only if they fulfill at least 1 of the following criteria:

- They induce clinical signs or symptoms

- They are considered clinically significant

- They require therapy

Clinically significant abnormal laboratory values or test results should be identified through a review of values outside of normal ranges/clinically notable ranges, significant changes from Baseline or the previous visit, or values which are considered to be nontypical in patient with underlying disease. Investigators have the responsibility for managing the safety of individual patients and identifying AEs.

Adverse events should be recorded in the Adverse Events eCRF under the signs, symptoms, or diagnosis associated with them, accompanied by the following information.

- Severity

- Mild: usually transient in nature and generally not interfering with normal activities

- Moderate: sufficiently discomforting to interfere with normal activities

- Severe: prevents normal activities

- Site (non-ocular/ocular) and laterality (bilateral/left eye/right eye), if an ocular AE

- Relationship to the study treatment (laser or ranibizumab) and to the study procedure (e.g., intravitreal injection)

- Duration (start and end dates) or if the event is ongoing an outcome of not recovered/not resolved or recovering/resolving should be reported 
- Whether it constitutes an SAE (see Section 7.2.1 for definition of an SAE)

- Action taken with study treatment (none/dose adjusted/temporarily interrupted/permanently discontinued/unknown/not applicable)

- Medication or therapies taken (no concomitant medication or nondrug therapy/concomitant medication or nondrug therapy)

- Outcome (not recovered/not resolved; recovered/resolved; recovering/resolving, recovered/resolved with sequelae; fatal; or unknown)

Once an AE is detected, it should be followed until its resolution or until it is judged to be permanent, and assessment should be made at each visit (or more frequently, if necessary) of any changes in severity, the suspected relationship to the investigational treatment, the interventions required to treat it, and the outcome.

Information about common side effects already known about the investigational drug can be found in the IB or will be communicated between IB updates in the form of investigator notifications. This information will be included in the patient informed consent and should be discussed with the patient's parent(s) or legal guardian(s) during the study as needed.

The Investigator should also instruct each patient's parent(s) or legal guardian(s) to report any new AE (beyond the protocol observation period) that the patient's parent(s) or legal guardian(s), or the patient's personal physician, believes might reasonably be related to the investigational treatment. This information should be recorded in the Investigator's source documents; however, if the AE meets the criteria of an SAE, it must be reported to Novartis.

\subsection{Serious adverse events}

\subsubsection{Definition of a serious adverse event}

An SAE is defined as any AE (appearance of (or worsening of any pre-existing) undesirable sign(s), symptom(s) or medical conditions(s)) which meets any 1 of the following criteria:

- Is fatal or life threatening

- Results in persistent or significant disability/incapacity

- Constitutes a congenital anomaly/birth defect

- Requires inpatient hospitalization or prolongation of existing hospitalization, unless hospitalization is for:

- Routine treatment or monitoring of the studied indication, not associated with any deterioration in condition

- Elective or preplanned treatment for a pre-existing condition that is unrelated to the indication under study and has not worsened since signing the informed consent

- Treatment on an emergency outpatient basis for an event not fulfilling any of the definitions of an SAE given above and not resulting in hospital admission

- Social reasons and respite care in the absence of any deterioration in the patient's general condition 
- Is medically significant, i.e., defined as an event that jeopardizes the patient or may require medical or surgical intervention

All malignant neoplasms will be assessed as serious under "medically significant" if other seriousness criteria are not met.

Life threatening in the context of an SAE refers to a reaction in which the patient was at risk of death at the time of the reaction; it does not refer to a reaction that hypothetically might have caused death if more severe (see Annex IV, International Conference on Harmonisation (ICH)-E2D Guideline).

Medical and scientific judgment should be exercised in deciding whether other situations should be considered serious reactions, such as important medical events that might not be immediately life threatening or result in death or hospitalization but might jeopardize the patient or might require intervention to prevent one of the other outcomes previously listed Examples of such events are intensive treatment in an emergency room or at home for allergic bronchospasm, blood dyscrasias or convulsions that do not result in hospitalization, or development of dependency or abuse (see Annex IV, ICH-E2D Guideline).

Any suspected transmission via a medicinal product of an infectious agent is also considered a serious adverse reaction.

In this study, the following safety events will be collected as study endpoints only, and are not to be considered or recorded as an AE or SAE:

- Substantial temporal retinal vessel dragging causing abnormal structural features/ macular ectopia

- Retrolental membrane obscuring the view of the posterior pole

- Posterior retinal fold involving the macula

- Retinal detachment

- Pre-retinal fibrosis

All serious and non-serious AEs (except those to be captured as study endpoints, as detailed above) are to be captured in the AE page of the eCRF; SAEs also require individual reporting to Novartis' Drug Safety and Epidemiology Department as per Section 7.2.2.

\subsubsection{SAE reporting}

To ensure patient safety, every SAE, regardless of causality, occurring after the patient's parent(s) or legal guardian(s) has provided informed consent and until 30 days after the last study visit or following the last administration of investigational treatment whichever is later must be reported to Novartis within 24 hours of learning of its occurrence. Any SAEs experienced after the 30 day period should only be reported to Novartis if the Investigator suspects a causal relationship to study treatment.

Recurrent episodes, complications, or progression of the initial SAE must be reported as follow-up to the original episode, regardless of when the event occurs. This report must be submitted within 24 hours of the Investigator receiving the follow-up information. An SAE that is considered completely unrelated to a previously reported one should be reported separately as a new event. 
Information about all SAEs (either initial or follow up information) is collected and recorded in English on the paper Serious Adverse Event Report Form or the electronic Serious Adverse Event Form within the Oracle Clinical/Remote Data Capture (OC/RDC) system (where available). The Investigator must assess the relationship to each specific component of the study treatment (if the study treatment consists of several components).

SAEs (initial and follow-up) that are recorded on the paper SAE form should be faxed within 24 hours of awareness of the SAE to the local Novartis Drug Safety and Epidemiology Department. The telephone and fax number of the contact persons in the local department of Drug Safety and Epidemiology, specific to the site, are listed in the investigator folder provided to each site. The original copy of the SAE Report Form and the fax confirmation sheet must be kept with the case report form documentation at the study site. Follow-up information should be provided using a new paper SAE Report Form stating that this is a follow-up to a previously reported SAE.

SAEs (initial and follow-up) that are recorded electronically in the OC/RDC system should be entered, saved and e-signed within 24 hours of awareness of the SAE or changes to an existing SAE. These data will automatically be submitted to Novartis Drug Safety \& Epidemiology immediately after investigator signature or 24 hours after entry, whichever occurs first.

Follow-up information provided should describe whether the event has resolved or continues, if and how it was treated and whether the patient continued or withdrew from study participation. Each re-occurrence, complication, or progression of the original event should be reported as a follow-up to that event regardless of when it occurs.

If the SAE is not previously documented in the IB or Package Insert (new occurrence) and is thought to be related to the investigational treatment a Drug Safety and Epidemiology Department associate may urgently require further information from the investigator for Health Authority reporting. Novartis may need to issue an Investigator Notification to inform all investigators involved in any study with the same investigational treatment that this SAE has been reported. Suspected Unexpected Serious Adverse Reactions will be collected and reported to the competent authorities and relevant ethics committees in accordance with European Union Guidance 2011/C 172/01 or as per national regulatory requirements in participating countries.

Safety events that are collected as study endpoints will not be considered as SAEs and do not follow the above expedited reporting process (Section 7.2.1). These events will be monitored and reviewed regularly by Novartis or designee and by the DMC.

\section{$8 \quad$ Data review and database management}

\subsection{Site monitoring}

Before study initiation, at a site initiation visit or at an Investigator's meeting, a Novartis representative will review the protocol and CRFs with the investigators and their staff. During the study, Novartis employs several methods of ensuring protocol and Good Clinical Practice (GCP) compliance and the quality/integrity of the sites' data. The field monitor will visit the 
site to check the completeness of patient records, the accuracy of entries in the eCRFs, the adherence to the protocol and to GCP, the progress of enrollment, and to ensure that study treatment is being stored, dispensed, and accounted for according to specifications. Key study personnel must be available to assist the field monitor during these visits. Continuous remote monitoring of each site's data may be performed by a centralized Novartis CRA organization. Additionally, a central analytics organization may analyze data, identify risks and trends for site operational parameters, and provide reports to Novartis Clinical Teams to assist with trial oversight.

The Investigator must maintain source documents for each patient in the study, consisting of case and visit notes (hospital or clinic medical records) containing demographic and medical information, laboratory data, electrocardiograms, and the results of any other tests or assessments. All information in the eCRFs must be traceable to these source documents in the patient's file. The Investigator must also keep the original informed consent form signed by the patient's parent(s) or legal guardian(s) (a signed copy is given to the patient's parent(s) or legal guardian(s)).

The Investigator must give the monitor access to all relevant source documents to confirm their consistency with the eCRF entries. Novartis' monitoring standards require full verification for the presence of informed consent, adherence to the inclusion/exclusion criteria, and documentation of SAEs and of data that will be used for all primary variables. Additional checks of the consistency of the source data with the eCRFs are performed according to the study-specific monitoring plan. No information in source documents about the identity of the patients will be disclosed.

\subsection{Data collection}

Designated investigator staff will enter the data required by the protocol into the OC/RDC system. Designated investigator site staff will not be given access to the system until they have been trained.

Automatic validation procedures within the system check for data discrepancies during and after data entry and, by generating appropriate error messages, allow the data to be confirmed or corrected online by the designated investigator site staff. The Investigator must certify that the data entered into the electronic Case Report Forms are complete and accurate. After database lock, the investigator will receive copies of the patient data for archiving at the investigational site.

\subsection{Database management and quality control}

Novartis staff will review the data entered into the CRFs by investigational staff for completeness and accuracy and instruct the site personnel to make any required corrections or additions. Queries are sent to the investigational site using an electronic data query. Designated investigator site staff is required to respond to the query and confirm or correct the data. If the electronic query system is not used, a paper Data Query Form will be faxed to the site. Site personnel will complete and sign the faxed copy and fax it back to Novartis staff that will make the correction to the database. The signed copy of the Data Query Form is kept at the investigator site. 
Concomitant medications entered into the database will be coded using the latest World Health Organization (WHO) Drug Reference List, which employs the Anatomical Therapeutic Chemical (ATC) classification system. Concomitant procedures, nondrug therapies, and AEs will be coded using the latest Medical Dictionary for Regulatory Activities (MedDRA) terminology.

Laboratory samples collected according to Sections 6.6.1, 6.6.2 and 6.6.3, respectively, will be processed centrally and the results will be sent electronically to Novartis. Samples taken as part of routine clinical practice according to Section 6.5.5, will be analyzed locally and results will be entered into the respective eCRFs by designated investigator site staff.

RetCam images will be processed by the Central Reading Center and the results will be sent electronically to Novartis.

Randomization codes and data about all study drug(s) dispensed to the patient will be tracked using an IRT. The system will be supplied by a vendor, who will also manage the database. The database will be sent electronically to Novartis.

The occurrence of relevant protocol deviations will be determined. After these actions have been completed and the database has been declared to be complete and accurate, it will be locked and the treatment codes will be unmasked and made available for data analysis. Although this is an open-label study, the CTT will not have access to randomization data until after database lock (Section 5.4). Any changes to the database after that time can only be made after written agreement by Novartis Development Management.

\subsection{Data Monitoring Committee}

An independent DMC will be established to monitor the safety of the trial participants, to ensure that the trial is being conducted with the highest scientific and ethical standards, and make appropriate recommendations based on the data seen.

The DMC charter will include the DMC membership and responsibilities, the timing of DMC meetings, the content of the analysis report for the DMC meetings, and the communication with the Sponsor. The DMC will only make recommendations for changes in study conduct.

\section{$9 \quad$ Data analysis}

The statistical analysis will be performed or directed by the biostatistician at Novartis.

At all of the time points assessed, continuous variables will be presented based on descriptive statistics (mean, standard deviation, median, minimal and maximum values) for the observed values and change from Baseline. Binary variables will be presented by percentage and exact 2-sided 95\% confidence interval. Graphical presentations providing 2-sided 95\% confidence intervals will be displayed as appropriate.

The baseline value is the last available, non-missing, value collected prior to Baseline (i.e., prior to receiving the first investigational treatment for treated patients). If a patient is randomized but not treated, then the baseline value for a variable is the last available nonmissing value collected prior to randomization. 
Further technical details and discussion of the following statistical considerations will be provided in the statistical analysis plan (SAP), which will be finalized before the database lock.

\subsection{Analysis sets}

The Screened Set will consist of all patients whose parent(s) or legal guardian(s) has provided informed consent.

The data from patients whose parent(s) or legal guardian(s) has not provided informed consent prior to any study procedures will be listed only and will not be used in any analyses.

The Randomized Set will consist of all randomized patients.

The Full Analysis Set (FAS) will comprise all patients from the Randomized Set to whom treatment regimen has been assigned. Following the intent-to-treat principle, patients will be analyzed for efficacy according to the treatment regimen they are assigned to at randomization.

The Per Protocol Set (PPS) will consist of all patients in the Full Analysis Set who follow the treatment regimen as randomized and complete the study period for the analysis of the primary and key secondary objectives without clinically important protocol deviations. Clinically important protocol deviations will be identified and documented prior to the database lock. The PPS will be used in a supportive analysis for the primary and key secondary efficacy analyses.

The Safety Set will consist of all patients who receive at least 1 application of investigational treatment and have at least 1 post baseline safety assessment. The statement that a patient has no AEs also constitutes a safety assessment.

The Randomized Set will be used to display patient disposition, demographics and baseline characteristics, protocol deviations, and medical history. The Full Analysis Set will be used for the primary analysis of the efficacy evaluation using the intent-to-treat principle. A patient will be considered to be evaluable if following imputation of missing data (Section 9.4.3) the patient provides data for the primary analysis. The Safety Set will be used for exposure, the safety evaluation, and to display concomitant and rescue medications.

\subsection{Patient demographics and other baseline characteristics}

Patient demographics and all baseline characteristics (including the baseline values of the main efficacy endpoints) will be summarized for each treatment group and for all patients (total).

Descriptive statistics (mean, median, standard deviation, minimum, and maximum) will be presented for continuous variables. The number and percentage of patients in each category defined in the trial SAP will be presented for categorical variables.

Relevant medical history and current medical conditions will be tabulated by system organ class (arranged alphabetically) and preferred term (arranged by decreasing frequency in the ranibizumab 0.2-mg treatment arm) of the latest version of MedDRA. Separate tables will be presented for ocular and non-ocular histories and conditions. 
For the purposes of classifying an eye by ROP disease status, the worst disease present in any clock hour of the eye, ranked in descending order from worse to best in Table 9-1, will be used to classify the eye at Baseline and during the study. Plus disease is defined as vascular tortuosity and dilatation in at least 2 quadrants of the eye (International Committee for the Classification of Retinopathy of Prematurity 2005).

Table 9-1 Ranking of Retinopathy of Prematurity Disease

\begin{tabular}{cc}
\hline Zone & Stage \\
\hline Any & 5 \\
Any & 4 \\
I & AP-ROP \\
II & AP-ROP \\
I & $3+$ \\
I & 3 \\
I & $2+$ \\
I & $1+$ \\
II & $3+$ \\
II & $2+$ \\
I & 2 \\
I & 1 \\
II & 3 \\
II & 2 \\
II & $1+$ \\
II & 1 \\
III & $3+$ \\
III & 3 \\
III & $2+$ \\
III & 2 \\
III & $1+$ \\
III & 1 \\
No ROP & No ROP
\end{tabular}

Abbreviations: AP-ROP - aggressive posterior retinopathy of prematurity; ROP - retinopathy of prematurity

For the purposes of classifying a patient by ROP disease status, the worst eye, ranked in descending order from worse to best in Table 9-1, will be used to classify the patient at Baseline. If both eyes have the same zone and stage, then the worst eye is the one with the greater total number of clock hours with the highest stage; if this is also the same for both eyes, than the left eye will be assigned as the worst eye.

Other relevant baseline information will be summarized as appropriate.

\subsection{Treatments}

Exposure to investigational treatment will be presented by treatment group. Concomitant therapies will be summarized by preferred term according to the latest version of the WHO 
Drug Reference List dictionary and ATC codes. Separate summaries will be presented for the time periods before and after the first investigational treatment. Concomitant therapies taken after the first investigational treatment, which are prohibited according to the protocol, will be summarized separately.

\subsection{Analysis of the primary variables}

\subsubsection{Variables}

The primary efficacy variable is the absence of active ROP and absence of unfavorable structural outcomes in both eyes 24 weeks after starting investigational treatment, as assessed by the Investigator. To achieve this outcome, patients cannot fulfill any of the following criteria:

- Death at or before the 24-week assessment visit

- Requires intervention for ROP in either eye at or before the 24-week assessment visit with a treatment modality other than the modality of the first investigational treatment

- Have active ROP in either eye at the 24-week assessment visit as defined by the presence of any of the following features:

- Vessel dilatation of plus disease in at least 2 quadrants of the eye (some persisting tortuosity is allowed)

- Extra-retinal vessels extending from the retina into the vitreous and judged to be a sign of active ROP disease

- Have unfavorable structural outcomes in either eye at or before the 24-week assessment visit as defined by the presence of any of the following features:

- Retrolental membrane obscuring the view of the posterior pole

- Substantial temporal retinal vessel dragging causing abnormal structural features/ macular ectopia

- Posterior retinal fold involving the macula

- Retinal detachment involving the macula

A patient may be defined as a "success" (favorable outcome) or "failure" (unfavorable outcome) for each of the criteria above. Patients will be defined as a "success" for the primary efficacy variable only if they are a "success" for each component. Consequently a patient will be defined as a "failure" for the primary efficacy variable if they are defined as a "failure" for at least one of the criteria above.

A 2 -sided family-wise type I error rate of $5 \%$ will be used. Two-sided exact $95 \%$ confidence intervals will also be presented for the primary variable event rate within each treatment arm.

\subsubsection{Statistical model, hypothesis, and method of analysis}

A three-step sequential testing procedure will be used for primary (ranibizumab $0.2 \mathrm{mg}$ against laser) and two key secondary comparisons (ranibizumab $0.1 \mathrm{mg}$ against laser and ranibizumab $0.2 \mathrm{mg}$ against $0.1 \mathrm{mg}$ ). Under this testing procedure, the primary comparison will be conducted at the first step followed sequentially by the two key secondary comparisons if the primary comparison is statistically significant. If the efficacy comparison 
at any step is not statistically significant, the remaining efficacy comparisons will be assessed descriptively. Otherwise the comparison will continue to the next step.

All hypotheses will be tested at a pre-specified level of significance (two sided $\alpha=0.05$ ). This testing procedure controls familywise type I error rate at a pre-specified level of significance because, for each hypothesis, testing is conditional upon rejecting all hypotheses earlier in the sequence.

The null hypotheses being tested at each step are:

Step 1: (primary comparison)

$\mathrm{H}_{01}: \pi$ Ranibizumab $0.2 \mathrm{mg}-\pi$ Laser $=0$

versus the alternative hypothesis

$\mathrm{H}_{\mathrm{A} 1}: \pi$ Ranibizumab $0.2 \mathrm{mg}-\pi_{\text {Laser }} \neq 0$

where $\pi$ Treatment arm is the unknown proportion of patients with absence of active ROP and absence of unfavorable structural outcomes in both eyes 24 weeks after starting investigational treatment, as assessed by the Investigator in the relevant treatment arm.

If hypothesis $\mathrm{H}_{01}$ is rejected at the 2-sided 5\% significance level and ranibizumab $0.2 \mathrm{mg}$ is concluded to be superior compared to laser then proceed to the next step. Otherwise stop and assess key secondary comparisons (ranibizumab $0.1 \mathrm{mg}$ against laser, and ranibizumab $0.2 \mathrm{mg}$ against $0.1 \mathrm{mg}$ ) descriptively.

Step 2: (first key secondary comparison)

$\mathrm{H}_{02}: \pi_{\text {Ranibizumab } 0.1 \mathrm{mg}}-\pi_{\text {Laser }}=0$

versus the alternative hypothesis

$\mathrm{H}_{\mathrm{A} 2}: \pi$ Ranibizumab $0.1 \mathrm{mg}-\pi$ Laser $\neq 0$

If hypothesis $\mathrm{H}_{02}$ is rejected at the 2-sided 5\% significance level and ranibizumab $0.1 \mathrm{mg}$ is concluded to be superior compared to laser then proceed to the next step. Otherwise stop and assess second key secondary comparison (ranibizumab $0.2 \mathrm{mg}$ against $0.1 \mathrm{mg}$ ) descriptively.

Step 3: (second key secondary comparison)

$\mathrm{H}_{03}: \pi$ Ranibizumab $0.2 \mathrm{mg}-\pi$ Ranibizumab $0.1 \mathrm{mg}=0$

versus the alternative hypothesis

$\mathrm{H}_{\mathrm{A} 3}: \pi$ Ranibizumab $0.2 \mathrm{mg}-\pi$ Ranibizumab $0.1 \mathrm{mg} \neq 0$

If ranibizumab $0.2 \mathrm{mg}$ is not concluded to be superior compared to $0.1 \mathrm{mg}$ or hypothesis $\mathrm{H}_{03}$ is accepted at the 2-sided 5\% significance level then the comparison between ranibizumab $0.2 \mathrm{mg}$ against $0.1 \mathrm{mg}$ will be assessed descriptively. 
The comparisons will be performed using the stratified Cochran-Mantel-Haenszel test for binomial proportions. Stratification will be based on ROP zone at baseline as used for randomization. Mantel-Haenszel odds ratios and their 95\% confidence intervals will also be presented.

Superiority of ranibizumab $0.2 \mathrm{mg}$ over laser will be concluded if hypothesis $\mathrm{H}_{01}$ is rejected (at the two-sided 5\% significance level) and Mantel-Haenszel odds ratio of ranibizumab $0.2 \mathrm{mg}$ to laser is in favor of ranibizumab $0.2 \mathrm{mg}$ with regard to the success of the primary efficacy variable.

Superiority of ranibizumab $0.1 \mathrm{mg}$ over laser will be concluded if hypothesis $\mathrm{H}_{02}$ is rejected (at the two-sided 5\% significance level), and Mantel-Haenszel odds ratio of ranibizumab $0.1 \mathrm{mg}$ to laser is in favor of ranibizumab $0.1 \mathrm{mg}$.

Superiority of ranibizumab $0.2 \mathrm{mg}$ over $0.1 \mathrm{mg}$ will be concluded if hypothesis $\mathrm{H}_{03}$ is rejected (at the two-sided 5\% significance level) and Mantel-Haenszel odds ratio of ranibizumab $0.2 \mathrm{mg}$ versus $0.1 \mathrm{mg}$ is in favor of ranibizumab $0.2 \mathrm{mg}$.

\subsubsection{Handling of missing values/censoring/discontinuations}

Imputation of missing data will only be undertaken if the value of the primary variable is missing. The primary analysis of the hypotheses in Section 9.4 .2 will be undertaken after imputing missing values of the primary variable at 24 weeks after the first investigational treatment using a 2-step single imputation approach.

In the first step, imputation of missing individual criteria related to the occurrence of active ROP and unfavorable structural outcomes at Week 24 will be undertaken assuming the following:

- If the last non-missing value of a criterion is "success", then the missing value of the criterion at Week 24 will stay missing

- If the last non-missing value of a criterion is "failure" then the missing value of the component at Week 24 will be imputed by "failure"

In the second step, the imputed value of the primary variable will then be derived from the imputed values of the individual components. The imputed value of the primary variable will be set to:

- Failure if at least one of the individual criteria is "failure".

- "Success" if all of the individual criteria are "success".

- Missing if at least one of the individual components is missing and none of the other components are "failure".

\subsubsection{Supportive analyses}

The robustness of the primary analysis (testing the hypotheses specified in Section 9.4.2) may be assessed by:

- Repeating the primary analyses using the PPS

- Repeating the primary analyses without imputing data missing at Week 24

- Repeating the primary analyses imputing data missing at Week 24 by "success" 
- Repeating the primary analyses imputing data missing at Week 24 by "failure"

- Replacing the stratified Cochran-Mantel-Haenszel test by using a logistic regression model using treatment arm and ROP zone at baseline as factors

- Adding prognostic variables to the logistic regression model previously defined

- Adding predictive variables to the logistic regression model previously defined

Geographic region will be included as a predictive factor. Other prognostic/predictive factors will be defined before first patient first visit. Each prognostic/predictive factor will be considered individually.

\subsection{Analysis of secondary and exploratory variables}

\subsubsection{Efficacy variables}

Secondary efficacy variables include:

- The criteria that constitute the definition of absence of active ROP and unfavorable ocular outcomes at 24 weeks after starting investigational treatment, as assessed by the Investigator.

- Death from any cause at or before the 24-week assessment visit

- Requirement of intervention for ROP in either eye at or before the 24-week assessment visit with a treatment modality other than the modality of the first investigational treatment

- Vessel dilatation of plus disease in at least 2 quadrants of the eye, in either eye (some persisting tortuosity is allowed) at the 24 -week assessment visit

- Presence of extra-retinal vessels extending from the retina into the vitreous and judged to be a sign of active ROP disease in either eye at the 24-week assessment visit

- Presence of retrolental membrane obscuring the view of the posterior pole in either eye at or before the 24-week assessment visit

- Substantial temporal retinal vessel dragging causing abnormal structural features/ macular ectopia in either eye at or before the 24-week assessment visit

- Posterior retinal fold involving the macula in either eye at or before the 24-week assessment visit

- Retinal detachment involving the macula in either eye at or before the 24-week assessment visit

- Any of the following unfavorable structural outcomes in either eye at or before 24 weeks after the first investigational treatment, as assessed by the Investigator: retrolental membrane obscuring the view of the posterior pole, substantial temporal retinal vessel dragging causing abnormal structural features/ macular ectopia, posterior retinal fold involving the macula, retinal detachment involving the macula

- The time after the first investigational treatment to the first occurrence of one of the following:

- Death 
- Intervention for ROP with a treatment modality other than the modality of the first investigational treatment

- An unfavorable structural outcome in either eye as defined by the presence of any of the following features:

- Retrolental membrane obscuring the view of the posterior pole

- Substantial temporal retinal vessel dragging causing abnormal structural features/ macular ectopia

- Posterior retinal fold involving the macula

- Retinal detachment involving the macula

- Requirement of none, 1,2 or 3 ranibizumab treatments in each eye by patient

- Recurrence of ROP receiving any post-baseline intervention at 24 weeks or before

Exploratory efficacy variables include:

- At or before 24 weeks after starting investigational treatment, as assessed by the Investigator:

- Retinal detachment not involving the macula in either eye

- Pre-retinal fibrosis in either eye

- The primary variable excluding the requirement for the intervention for ROP in either eye at or before the 24 -week assessment visit with a treatment modality other than the modality of the first investigational treatment

- At 24 weeks after starting investigational treatment, as assessed by the Investigator:

- Full retinal vascularization in 12 clock hours

No imputation of missing data will be undertaken on exploratory efficacy variables. No statistical inference tests will be undertaken on exploratory efficacy variables.

Descriptive statistics will be produced for binary efficacy variables. The proportion of patients and the proportion of eyes satisfying the condition (e.g., those that have evidence of vessel dilatation of plus disease) will be given by treatment arm.

Exact 2-sided 95\% confidence intervals will be given for the proportion of patients satisfying the binary efficacy variable condition by treatment arm. Kaplan-Meier plots will be provided for time to event variables.

\subsubsection{Safety variables}

Safety analyses will be conducted on data from Safety set. Adverse events, vital signs measurements, requirement for respiratory support, the presence of fundus features abnormalities and laboratory evaluations will be summarized and listed.

Adverse events will be deemed treatment emergent if the onset date is on or after the date of first investigational treatment. Any AEs recorded prior to the start of investigational treatment will be listed only.

Only treatment-emergent AEs will be summarized by presenting, for each treatment arm and overall, the number and percentage of patients having any AE, having any ocular AE, and having any systemic AE. Adverse events will be presented by primary system organ class 
(arranged alphabetically) and preferred term (arranged by decreasing frequency in the ranibizumab $0.2 \mathrm{mg}$ treatment arm) using the most recent version of MedDRA available. Patients who experienced multiple AEs for a preferred term will be counted once, similarly for patients with multiple AEs per system organ class.

All other information collected (e.g., severity, relationship to study procedures and investigational treatment [ranibizumab/laser]) will be tabulated and listed as appropriate.

Deaths, SAEs, and AEs leading to permanent discontinuation of investigational treatment will be listed separately and, if appropriate, summarized by primary system organ class and preferred term.

Vital signs, requirement for respiratory support and laboratory evaluations will be summarized by presenting descriptive statistics of raw values and change from baseline by visit and treatment group. Shift tables will be used, as appropriate, to compare baseline and post-baseline categorical safety parameters. Other safety data will be summarized and listed as appropriate.

\subsubsection{Ocular and visual function}

The presence of nystagmus, abnormal fixation behavior, and abnormal pupillary light reaction at 24 weeks will be summarized and tabulated.

Refractive error at 24 weeks will be categorized into myopia (spherical equivalent of 0.25 diopters or more of myopia) and high myopia (spherical equivalent of 5.00 diopters or more of myopia). The proportion of patients without myopia, with myopia, and with high myopia will be summarized and tabulated by treatment arm.

\subsubsection{Systemic ranibizumab concentration}

All patients providing pharmacokinetic serum samples will be included in the PK analysis population. Individual ranibizumab concentrations versus time profiles will be displayed graphically. Ranibizumab concentration-time data will be evaluated by population PK methods to describe ranibizumab exposure and will be compared to exposure in adults from previous studies.

\subsubsection{Pharmacogenetics/pharmacogenomics}

Not applicable.

\subsubsection{Systemic vascular endothelial growth factor level}

All patients providing VEGF plasma samples will be included in the VEGF analysis population.

The detailed VEGF concentration-time pattern after ranibizumab administration in neonates is unknown. If possible, VEGF concentration-time profiles will be evaluated by population concentration-response methods (e.g., an indirect-response model) and compared to the concentration-time pattern in patients in the control treatment group. 


\subsubsection{Systemic anti-ranibizumab antibody level}

All patients with sufficient serum sample for anti-ranibizumab antibody testing will be included in the anti-ranibizumab antibody analysis population. The presence or absence of anti-ranibizumab antibodies will be summarized by ranibizumab dose and time point. In addition, switch tables will be used to summarize the change during the assessment time points.

\subsubsection{Hospitalization}

The duration of hospitalization and the weight at hospital discharge will be summarized and tabulated.

\subsection{Interim analyses}

There will be no interim analyses during the trial.

\subsection{Sample size calculation}

The Cochran-Mantel Haenszel test stratified by ROP zone will be used for hypothesis testing since the primary variable event rate is expected to vary according to whether the patient has ROP in zone I or zone II. The proportion of patients with this event is assumed to be similar to that in the BEAT-ROP study for the recurrence of ROP at 54 weeks postmenstrual age (Mintz-Hittner et al 2011):

- Zone I

- Laser $-42.4 \%$

- Ranibizumab $0.2 \mathrm{mg}-6.5 \%$

- Zone II

- $\quad$ Laser $-12.5 \%$

- Ranibizumab $0.2 \mathrm{mg}-5.1 \%$

Monte Carlo simulations were conducted with the following assumptions:

- 10000 trials

- Data from 48 patients were evaluable (non-missing for the primary analysis following imputation) in each treatment arm (144 total evaluable)

- Event rates as previously shown

- Each patient had a $45 \%$ and $55 \%$ probability of ROP in zones I and II, respectively

- The treatment effect of the ranibizumab $0.1 \mathrm{mg}$ was half that of ranibizumab $0.2 \mathrm{mg}$

It is estimated that the proposed three-step sequential design with the assumptions described in Section 9.4.2 will yield more than $80 \%$ power to show superiority of ranibizumab $0.2 \mathrm{mg}$ against laser therapy (primary objective of the study) in the treatment of ROP. Sixty patients are planned to be randomized per arm in this study. With an estimated drop-out rate of $20 \%$, at least 48 patients are expected to provide data for primary and key-secondary efficacy analyses. 


\section{$10 \quad$ Ethical considerations}

\subsection{Regulatory and ethical compliance}

This clinical study was designed and shall be implemented and reported in accordance with the ICH harmonised tripartite guidelines for GCP with applicable local regulations (including European Directive 2001/20/EC, US 21 CFR, and Japanese Ministry of Health, Labor, and Welfare), and with the ethical principles laid down in the Declaration of Helsinki.

\subsection{Informed consent procedures}

An eligible patient may only be included in the study after his/her parent(s) or legal guardian(s) has provided written (witnessed, where required by law or regulation), IRB/IEC approved informed consent. Informed consent must be obtained before conducting any study-specific procedures (i.e., all of the procedures described in the protocol). The process of obtaining informed consent should be documented in the patient source documents.

Novartis will provide to Investigators in a separate document a proposed informed consent form that complies with the ICH GCP guideline and regulatory requirements and is considered appropriate for this study. Any changes to the proposed consent form suggested by the Investigator must be agreed to by Novartis before submission to the IRB/IEC, and a copy of the approved version must be provided to the Novartis monitor after IRB/IEC approval.

\subsection{Responsibilities of the Investigator and IRB/IEC}

Before initiating a trial, the Investigator/institution must obtain approval/favorable opinion from the IRB/IEC for the trial protocol, written informed consent form, consent form updates, patient recruitment procedures (e.g., advertisements), and any other written information to be provided to patients. Prior to study start, the Investigator is required to sign a protocol signature page confirming his/her agreement to conduct the study in accordance with these documents and all of the instructions and procedures found in this protocol and to give access to all relevant data and records to Novartis monitors, auditors, Novartis Quality Assurance representatives, designated agents of Novartis, IRBs/IECs, and regulatory authorities as required. If an inspection of the clinical site is requested by a regulatory authority, the Investigator must inform Novartis immediately that this request has been made.

\subsection{Publication of study protocol and results}

Novartis assures that the key design elements of this protocol will be posted in a publicly accessible database such as clinicaltrials.gov. In addition, upon study completion and finalization of the study report the results of this trial will be either submitted for publication and/or posted in a publicly accessible database of clinical trial results.

\section{Protocol adherence}

This protocol defines the study objectives, the study procedures, and the data to be collected on study participants. Additional assessments required to ensure safety of patients should be administered as deemed necessary on a case by case basis. Under no circumstances should an 
Investigator collect additional data or conduct any additional procedures for any research related purpose involving any investigational drugs.

Investigators ascertain they will apply due diligence to avoid protocol deviations. If an Investigator feels a protocol deviation would improve the conduct of the study this must be considered a protocol amendment, and unless such an amendment is agreed upon by Novartis and approved by the IRB/IEC and health authorities, where required, it cannot be implemented. All significant protocol deviations will be recorded and reported in the clinical study report.

\subsection{Protocol amendments}

Any change or addition to the protocol can only be made in a written protocol amendment that must be approved by Novartis, Health Authorities where required, and the IRB/IEC prior to implementation. Only amendments that are intended to eliminate an apparent immediate hazard to patients may be implemented immediately provided the Health Authorities are subsequently notified by protocol amendment and the reviewing IRB/IEC is notified. Notwithstanding the need for approval of formal protocol amendments, the Investigator is expected to take any immediate action required for the safety of any patient included in this study, even if this action represents a deviation from the protocol. In such cases, the reporting requirements identified in Section 7 Safety Monitoring should be followed.

\section{References}

References are available upon request.

Adkins B, Leclerc C, Marshall-Clarke S [2004] Neonatal adaptive immunity comes of age. Nat Rev Immunol; 4(7):553-64.

Ahmed AE, Channa R, Durrani J, et al [2010] Early experience with intravitreal bevacizumab combined with laser treatment for retinopathy of prematurity. Middle East Afr J Ophthalmol; 17(3):264-7.

Altinsoy HI, Mutlu FM, Gungor R, Sarici SU [2010] Combination of laser photocoagulation and intravitreal bevacizumab in aggressive posterior retinopathy of prematurity. Ophthalmic Surg Lasers Imaging; Mar 9:1-5.

Benesch M, Windelberg M, Sauseng W, et al [2008] Compassionate use of bevacizumab (Avastin) in children and young adults with refractory or recurrent solid tumors. Ann Oncol; 19(4):807-13.

Boothe RG, Dobson V, Teller DY [1985] Postnatal development of vision in human and nonhumans primates. Ann Rev Neurosci; 8:495-545.

Castellanos MA, Schwartz S, García-Aguirre G, et al [2013] Short-term outcome after intravitreal ranibizumab injections for the treatment of retinopathy of prematurity. $\mathrm{Br} \mathrm{J}$ Ophthalmol; 97(7):816-9.

Chung EJ, Kim JH, Ahn HS, et al [2007] Combination of laser photocoagulation and intravitreal bevacizumab (Avastin) for aggressive zone I retinopathy of prematurity. Graefes Arch Clin Exp Ophthalmol; 245(11):1727-30. 
Connolly BP, Ng EY, McNamara JA, et al [2002] A comparison of laser photocoagulation with cryotherapy for threshold retinopathy of prematurity at 10 years: Part 2. Refractive outcome. Ophthalmology; 109(5):936-41.

Cryotherapy for Retinopathy of Prematurity Cooperative Group [1988] Multicenter trial of cryotherapy for retinopathy of prematurity. Preliminary results. Arch Ophthalmol; 106(4):471-9.

Darlow BA, Ells AL, Gilbert CE, et al [2013] Are we there yet? Bevacizumab therapy for retinopathy of prematurity. Arch Dis Child Fetal Neonatal Ed; 98(2):F170-4.

De Pasquale MD, Pessolano R, Boldrini R, et al [2011] Continuing response to subsequent treatment lines with tyrosine kinase inhibitors in an adolescent with metastatic renal cell carcinoma. J Pediatr Hematol Oncol; 33(5):e176-9.

De Vriese AS, Tilton RG, Elger M, et al [2001] Antibodies against vascular endothelial growth factor improve early renal dysfunction in experimental diabetes. J Am Soc Nephrol; 12(5):993-1000.

Dorta P, Kychenthal A [2010] Treatment of type 1 retinopathy of prematurity with intravitreal bevacizumab (Avastin). Retina; 30(4 Suppl):S24-31.

Edward DP, Kaufman LM [2003] Anatomy, development, and physiology of visual system. Pediatr. Clin North Am; 50(1):1-23.

Eremina V, Cui S, Gerber H, et al [2006] Vascular endothelial growth factor a signaling in the podocyte-endothelial compartment is required for mesangial cell migration and survival. J Am Soc Nephrol; 17(3):724-35.

Eremina V, Jefferson JA, Kowalewska J, et al [2008] VEGF inhibition and renal thrombotic microangiopathy. N Engl J Med; 358(11):1129-36.

European Medicines Agency (EMEA) [2006] Avastin: EPAR - scientific discussion. 24 Jan 2006. Available from:

http://www.ema.europa.eu/docs/en_GB/document_library/EPAR_-

Scientific_Discussion/human/000582/WC500029262.pdf(Accessed 17 Sep 2014)

European Medicines Agency (EMEA) [2013] Eylea: EPAR - public assessment report. 21 Feb 2013. Available from:

http://www.ema.europa.eu/docs/en_GB/document library/EPAR_-

_Public_assessment_report/human/002392/WC500135744.pdf (Accessed 17 Sep 2014)

Fierson WM, American Academy of Pediatrics Section on Ophthalmology, American Academy of Ophthalmology, American Association for Pediatric Ophthalmology and Strabismus [2013] Screening examination of premature infants for retinopathy of prematurity. Pediatrics; 131(1):189-95.

Geloneck MM, Chuang AZ, Clark WL, et al [2014] Refractive outcomes following bevacizumab monotherapy compared with conventional laser treatment: a randomized clinical trial. JAMA Ophthalmol; Published online August 07, 2014 (doi:10.1001/jamaophthalmol.2014.2772) 
Gerber HP, Hillan KJ, Ryan AM, et al [1999] VEGF is required for growth and survival in neonatal mice. Development; 126(6):1149-59.

Gilbert C [2008] Retinopathy of prematurity: a global perspective of the epidemics, population of babies at risk and implications for control. Early Hum Dev; 84(2):77-82.

Glade Bender JL, Adamson PC, Reid JM, et al [2008] Phase I trial and pharmacokinetic study of bevacizumab in pediatric patients with refractory solid tumors: a Children's Oncology Group Study. J Clin Oncol; 26(3):399-405.

Good WV, Early Treatment for Retinopathy of Prematurity Cooperative Group [2004] Final results of the Early Treatment for Retinopathy of Prematurity (ETROP) randomized trial. Trans Am Ophthalmol Soc; 102:233-48.

Harder BC, von Baltz S, Jonas JB, et al [2011] Intravitreal bevacizumab for retinopathy of prematurity. J Ocul Pharmacol Ther; 27(6):623-7.

Hardy RJ, Good WV, Dobson V, et al [2004] Multicenter trial of early treatment for retinopathy of prematurity: study design. Control Clin Trials; 25(3):311-25.

Hartnett ME, Penn JS [2012] Mechanisms and management of retinopathy of prematurity. N Engl J Med; 367(26):2515-26.

Hoerster R, Muether P, Dahlke C, et al [2013] Serum concentrations of vascular endothelial growth factor in an infant treated with ranibizumab for retinopathy of prematurity. Acta Ophthalmol; 91(1):e74-5.

Honda S, Hirabayashi H, Tsukahara Y, et al [2008] Acute contraction of the proliferative membrane after an intravitreal injection of bevacizumab for advanced retinopathy of prematurity. Graefes Arch Clin Exp Ophthalmol; 246(7):1061-3.

Houston SK, Wykoff CC, Berrocal AM, et al [2013] Laser treatment for retinopathy of prematurity. Lasers Med Sci; 28(2):683-92.

$\mathrm{Hu}$ J, Blair MP, Shapiro MJ, et al [2012] Reactivation of retinopathy of prematurity after bevacizumab injection. Arch Ophthalmol; 130(8):1000-6.

International Committee for the Classification of Retinopathy of Prematurity [2005] The international classification of retinopathy of prematurity revisited. Arch Ophthalmol; 123(7):991-9.

Jang SY, Choi KS, Lee SJ [2010] Delayed-onset retinal detachment after an intravitreal injection of ranibizumab for zone 1 plus retinopathy of prematurity. J AAPOS; 14(5):457-9.

Jin K, Zhu Y, Sun Y, et al [2002] Vascular endothelial growth factor (VEGF) stimulates neurogenesis in vitro and in vivo. Proc Natl Acad Sci USA; 99(18):11946-50.

Kiely PM, Gillard-Crewther S, Nathan J, et al [1987] A comparison of ocular development of the Cynomolgus monkey and man. Clin Vision Sci; 1(3):269-80.

Kim J, Kim SJ, Chang YS, et al [2014] Combined intravitreal bevacizumab injection and zone I sparing laser photocoagulation in patients with zone I retinopathy of prematurity. Retina; 34(1):77-82. 
Kitamoto Y, Tokunaga H, Tomita K [1997] Vascular endothelial growth factor is an essential molecule for mouse kidney development: glomerulogenesis and nephrogenesis. J Clin Invest; 99(10):2351-7.

Kusaka S, Shima C, Wada K, et al [2008] Efficacy of intravitreal injection of bevacizumab for severe retinopathy of prematurity: a pilot study. Br J Ophthalmol; 92(11):1450-5.

Lalwani GA, Berrocal AM, Murray TG, et al [2008] Off-label use of intravitreal bevacizumab (Avastin) for salvage treatment in progressive threshold retinopathy of prematurity. Retina; 28(3 Suppl):S13-8.

Law JC, Recchia FM, Morrison DG, et al [2010] Intravitreal bevacizumab as adjunctive treatment for retinopathy of prematurity. J AAPOS; 14(1):6-10.

Lee JY, Chae JB, Yang SJ, et al [2010] Effects of intravitreal bevacizumab and laser in retinopathy of prematurity therapy on the development of peripheral retinal vessels. Graefes Arch Clin Exp Ophthalmol; 248(9):1257-62.

Lee SJ, Kim ET, Yoo BC, et al [2011] Plasma level of vascular endothelial growth factor in retinopathy of prematurity after intravitreal injection of bevacizumab (E-abstract). Invest Ophthalmol Vis Sci; 52:3165A. Available from:

http://abstracts.iovs.org//cgi/content/abstract/52/6/3165?sid=eb0ddfe6-4592-4281-aeb5974ff09f645a

Lin CJ, Chen SN, Hwang JF [2012] Intravitreal ranibizumab as salvage therapy in an extremely low-birth-weight infant with rush type retinopathy of prematurity. Oman J Ophthalmol; 5(3):184-6.

Malik AK, Beldwin ME, Peale F, et al [2006] Redundant roles of VEGF-B and PlGF during selective VEGF-A blockade in mice. Blood; 107(2):550-7. Micieli JA, Surkont M, Smith AF [2009] A systematic analysis of the off-label use of bevacizumab for severe retinopathy of prematurity. Am J Ophthalmol; 148(4):536-43.

Mintz-Hittner HA, Kennedy KA, Chuang AZ, et al [2011] Efficacy of intravitreal bevacizumab for stage 3+ retinopathy of prematurity. N Engl J Med; 364(7):603-15.

Mintz-Hittner HA, Kuffel RR Jr [2008] Intravitreal injection of bevacizumab (Avastin) for treatment of stage 3 retinopathy of prematurity in zone I or posterior zone II. Retina; 28(6):831-8.

Mota A, Carneiro A, Breda J, et al [2012] Combination of intravitreal ranibizumab and laser photocoagulation for aggressive posterior retinopathy of prematurity. Case Rep Ophthalmol; 3(1):136-41.

Nazari H, Modarres M, Parvaresh MM, et al [2010] Intravitreal bevacizumab in combination with laser therapy for the treatment of severe retinopathy of prematurity (ROP) associated with vitreous or retinal hemorrhage. Graefes Arch Clin Exp Ophthalmol; 248(12):1713-8.

Ng EY, Connolly BP, McNamara JA, et al [2002] A comparison of laser photocoagulation with cryotherapy for threshold retinopathy of prematurity at 10 years: Part 1 . Visual function and structural outcome. Ophthalmology; 109(5):928-34. 
Palmer EA, Hardy RJ, Dobson V et al [2005] 15-year outcomes following threshold retinopathy of prematurity: Final results from the multicenter trial of cryotherapy for retinopathy of prematurity. Arch Ophthalmol; 123(3):311-8.

Qiao-Grider Y, Hung LF, Kee CS, et al [2007] Normal ocular development in young rhesus monkeys (Macaca mulatta). Vision Res; 47(11):1424-44.

Quiroz-Mercado H, Martinez-Castellanos MA, Hernandez-Rojas ML, et al [2008] Antiangiogenic therapy with intravitreal bevacizumab for retinopathy of prematurity. Retina; 28(3 Suppl):S19-25.

Royal College of Paediatrics and Child Health, Royal College of Ophthalmologists, British Association of Perinatal Medicine, BLISS [2008] Guideline for the Screening and Treatment of Retinopathy of Prematurity. UK Retinopathy of Prematurity Guideline. May 2008.

Ryan AM, Eppler DB, Hagler KE, et al [1999] Preclinical safety evaluation of rhuMAb VEGF, an antiangiogenic humanized monoclonal antibody. Toxicol Pathol; 27(1):78-86.

Şahin A, Şahin M, Cingü AK, et al [2013] Intravitreal bevacizumab monotherapy for retinopathy of prematurity. Pediatr Int; 55(5):599-603.

Sato T, Kusaka S, Shimojo H, et al [2009] Vitreous levels of erythropoietin and vascular endothelial growth factor in eyes with retinopathy of prematurity. Ophthalmology; 116(9):1599-603.

Sato T, Wada K, Arahori H, et al [2012] Serum concentrations of bevacizumab (Avastin) and vascular endothelial growth factor in infants with retinopathy of prematurity. Am J Ophthalmol; 153(2):327-33.

Smith AR, Hennessy JM, Kurth MA, et al [2008] Reversible skeletal changes after treatment with bevacizumab in a child with cutaneovisceral angiomatosis with thrombocytopenia syndrome. Pediatr Blood Cancer; 51(3):418-20.

Smith LE [2008] Through the eyes of a child: understanding retinopathy through ROP the Friedenwald lecture. Invest Ophthalmol Vis Sci; 49(12):5177-82.

Sonmez K, Drenser KA, Capone A Jr, et al [2008] Vitreous levels of stromal cell-derived factor 1 and vascular endothelial growth factor in patients with retinopathy of prematurity. Ophthalmology; 115(6):1065-70.

Travassos A, Teixeira S, Ferreira P, et al [2007] Intravitreal bevacizumab in aggressive posterior retinopathy of prematurity. Ophthalmic Surg Lasers Imaging; 38(3):233-7.

Wu WC, Kuo HK, Yeh PT, et al [2013] An updated study of the use of bevacizumab in the treatment of patients with prethreshold retinopathy of prematurity in Taiwan. Am J Ophthalmol; 155(1):150-8.

Wu WC, Yeh PT, Chen SN, et al [2011] Effects and complications of bevacizumab use in patients with retinopathy of prematurity: a multicenter study in Taiwan. Ophthalmology; 118(1):176-83. 
Zepeda-Romero LC, Liera-Garcia JA, Gutierrez-Padilla JA, et al [2010] Paradoxical vascular-fibrotic reaction after intravitreal bevacizumab for retinopathy of prematurity. Eye (Lond); 24(5):931-3.

Zhou Y, Jiang Y, Bai Y, et al [2016] Vascular endothelial growth factor plasma levels before and after treatment of retinopathy of prematurity with ranibizumab. Graefes Arch Clin Exp Ophthalmol; 254:31-6. 
13 Appendix 1: Blood log for systemic ranibizumab / antiranibizumab antibody samples collection

Table 13-1 Time schedule for blood sampling for systemic ranibizumab / anti ranibizumab antibody

\begin{tabular}{|c|c|c|c|}
\hline Study day & Dose Ref ID & Sample ID & Blood volume $(\mathrm{mL})$ \\
\hline $\begin{array}{l}\text { Day } 1 \text { (within } 24 \mathrm{~h} \text { after the } 1^{\text {st }} \\
\text { administration of ranibizumab) }\end{array}$ & 1 & 101 & 0.8 (target), 0.5 (minimum) \\
\hline $\begin{array}{l}\text { Day } 15^{\mathrm{a}}\left(7-21 \text { days after the } 1^{\text {st }}\right. \\
\text { administration of ranibizumab) }\end{array}$ & 1 & 102 & 0.8 (target), 0.5 (minimum) \\
\hline $\begin{array}{l}\text { Day } 29^{a} \text { ( } 22-28 \text { days after the } 1^{\text {st }} \\
\text { administration of ranibizumab) }\end{array}$ & 1 & 103 & 0.8 (target), 0.5 (minimum) \\
\hline
\end{tabular}

\section{Appendix 2: Blood log for systemic VEGF samples collection}

Table 14-1 Time schedule for blood sampling for VEGF

\begin{tabular}{|c|c|c|c|}
\hline Study day & $\begin{array}{l}\text { Dose Ref } \\
\text { ID }\end{array}$ & $\begin{array}{l}\text { Sample } \\
\text { ID }\end{array}$ & Blood volume $(\mathrm{mL})$ \\
\hline $\begin{array}{l}\text { Day } 1 \text { (before the } 1^{\text {st }} \text {-administration of } \\
\text { investigational treatment) }\end{array}$ & 1 & 201 & 0.8 (target), 0.5 (minimum) \\
\hline $\begin{array}{l}\text { Day } 15^{\text {a }}\left(7-21 \text { days after the } 11^{\text {st }}\right. \\
\text { administration of investigational treatment) }\end{array}$ & 1 & 202 & 0.8 (target), 0.5 (minimum) \\
\hline $\begin{array}{l}\text { Day } 29^{a} \text { ( } 22-28 \text { days after the } 1^{\text {st }} \\
\text { administration of investigational treatment) }\end{array}$ & 1 & 203 & 0.8 (target), 0.5 (minimum) \\
\hline
\end{tabular}

